\title{
On second-order differentiability with respect to parameters for differential equations with state-dependent delays*
}

\author{
Ferenc Hartung \\ Department of Mathematics \\ University of Pannonia \\ 8201 Veszprém, P.O. Box 158, Hungary
}

November 18, 2018

\begin{abstract}
In this paper we consider a class of differential equations with state-dependent delays. We show first and second-order differentiability of the solution with respect to parameters in a pointwise sense and also using the $C$-norm on the state-space, assuming that the statedependent time lag function is piecewise strictly monotone.
\end{abstract}

AMS(MOS) subject classification: 34K05

keywords: Delay differential equation, state-dependent delay, differentiability with respect to parameters.

\section{Introduction}

In this paper we study the SD-DDE

$$
\dot{x}(t)=f\left(t, x_{t}, x\left(t-\tau\left(t, x_{t}, \xi\right)\right), \theta\right), \quad t \in[0, T],
$$

and the corresponding initial condition

$$
x(t)=\varphi(t), \quad t \in[-r, 0] .
$$

Let $\Theta$ and $\Xi$ be normed linear spaces with norms $|\cdot|_{\Theta}$ and $|\cdot|_{\Xi}$, respectively, and suppose $\theta \in \Theta$ and $\xi \in \Xi$. Here we consider the initial function $\varphi, \theta$ and $\xi$ as parameters in the IVP (1.1)-(1.2), and we denote the corresponding solution by $x(t, \varphi, \theta, \xi)$. The main goal of this paper is to discuss the differentiability of $x(t, \varphi, \theta, \xi)$ wrt $\varphi, \theta$ and $\xi$. By differentiability we mean Fréchet-differentiability throughout the manuscript. Differentiability of solutions wrt parameters is an important qualitative question, but it also has a natural application in the problem of identification of parameters (see [10]). But even for simple constant delay equations this problem leads to technical difficulties if the parameter is the delay [6, 17]. Similar difficulty arises in SD-DDEs.

\footnotetext{
*This research was partially supported by the Hungarian National Foundation for Scientific Research Grant
} No. K73274. 
Theorem 3.1 below yields that, under natural assumptions, Lipschitz continuous initial functions generate unique solutions of (1.1). As it is common for delay equations, as the time increases, the solution of (1.1) gets smoother wrt the time: on the interval $[0, r]$ the solution is $C^{1}$, on $[r, 2 r]$ it is a $C^{2}$ function, etc. But for $t \in[0, r]$ the solution segment function $x_{t}$ is only Lipschitz continuous. Therefore the linearization of the composite function $x\left(t-\tau\left(t, x_{t}, \xi\right)\right)$ is not straightforward, which is clearly needed at some point of the proof to obtain differentiability wrt parameters.

To illustrate the difficulty of this problem in the case when we can't assume continuous differentiability of $x$, we recall a result of Brokate and Colonius [1]. They studied SD-DDEs of the form

$$
x^{\prime}(t)=f(t, x(t-\tau(t, x(t)))), \quad t \in[a, b],
$$

and investigated differentiability of the composition operator

$$
A: W^{1, \infty}([a, b] ; \mathbb{R}) \supset \bar{X} \rightarrow L^{p}([a, b] ; \mathbb{R}), \quad A(x)(t):=x(t-\tau(t, x(t))) .
$$

They assumed that $\tau$ is twice continuously differentiable satisfying $a \leq t-\tau(t, v) \leq b$ for all $t \in[a, b]$ and $v \in \mathbb{R}$, and considered as domain of $A$ the set

$$
\bar{X}:=\left\{x \in W^{1, \infty}([a, b] ; \mathbb{R}): \text { There exists } \varepsilon>0 \text { s.t. } \frac{d}{d t}(t-\tau(t, x(t))) \geq \varepsilon \text { for a.e. } t \in[a, b]\right\} .
$$

It was shown in [1] that under these assumptions $A$ is continuously differentiable with the derivative given by

$$
(D A(x) u)(t)=-\dot{x}(t-\tau(t, x(t))) D_{2} \tau(t, x(t)) u(t)+u(t-\tau(t, x(t)))
$$

for $u \in W^{1, \infty}([a, b], \mathbb{R})$. Both the strong $W^{1, \infty}$-norm on the domain and the weak $L^{p}$-norm on the range, together with the choice of the domain seemed to be necessary to obtain the results in [1]. Note that Manitius in [18] used a similar domain and norm when he studied linearization for a class of SD-DDEs.

Differentiability of solutions wrt parameters for SD-DDEs was studied in $[2,9,12,16,21,22]$. In [9] differentiability of the parameter map was established at parameter values where the compatibility condition

$$
\varphi \in C^{1}, \quad \dot{\varphi}(0-)=f(0, \varphi, \varphi(-\tau(0, \varphi, \xi)), \theta)
$$

is satisfied. It was proved that the parameter map is differentiable in a pointwise sense, i.e., the map

$$
W^{1, \infty} \times \Theta \times \Xi \rightarrow \mathbb{R}^{n}, \quad(\varphi, \theta, \xi) \mapsto x(t, \varphi, \theta, \xi)
$$

is differentiable for every fixed $t$ from the domain of the solution. Moreover, it was shown that the map

$$
W^{1, \infty} \times \Theta \times \Xi \rightarrow C, \quad(\varphi, \theta, \xi) \mapsto x_{t}(\cdot, \varphi, \theta, \xi),
$$

and, under a little more smoothness assumptions, the map

$$
W^{1, \infty} \times \Theta \times \Xi \rightarrow W^{1, \infty}, \quad(\varphi, \theta, \xi) \mapsto x_{t}(\cdot, \varphi, \theta, \xi)
$$

is also differentiable at fixed parameter values satisfying (1.3). Note that a condition similar to (1.3) was used by Walter in [21] and [22], where proved the existence of a $C^{1}$-smooth solution semiflow for large classes of SD-DDES. 
In [16] differentiability of the parameter map was proved without assuming the compatibility condition (1.3). Instead, it was assumed that the time lag function $t \mapsto t-\tau\left(t, x_{t}, \xi\right)$ corresponding to a fixed solution $x$ is strictly monotone increasing, more precisely,

$$
\underset{0 \leq t \leq \alpha}{\operatorname{essinf}} \frac{d}{d t}\left(t-\tau\left(t, x_{t}, \xi\right)\right)>0,
$$

where $\alpha>0$ is such that the solution exists on $[-r, \alpha]$. Also, instead of a "pointwise" differentiability, the differentiability of the map

$$
W^{1, \infty} \times \Theta \times \Xi \rightarrow W^{1, p}, \quad(\varphi, \theta, \xi) \mapsto x_{t}(\cdot, \varphi, \theta, \xi)
$$

was proved in a small neighborhood of the fixed parameter value. Note that here the differentiability was obtained using only a weak norm, the $W^{1, p}$-norm $(1 \leq p<\infty)$ on the state-space.

Chen, $\mathrm{Hu}$ and $\mathrm{Wu}$ in [2] extended the above result to proving second ordered differentiability of the parameter map using the monotonicity condition (1.7) of the state-dependent time lag function, the $W^{1, p}$-norm $(1 \leq p<\infty)$ on the state space, and the $W^{2, p}$-norm on the space of initial functions. Note that $\tau$ was not given explicitly in [2], it was defined through a coupled differential equation, but it satisfied the monotonicity condition (1.7).

In $[12]$ the IVP

$$
\begin{aligned}
& \dot{x}(t)=f\left(t, x_{t}, x\left(t-\tau\left(t, x_{t}\right)\right)\right), \quad t \in[\sigma, T], \\
& x(t)=\varphi(t-\sigma), \quad t \in[\sigma-r, \sigma]
\end{aligned}
$$

was considered. In this IVP the parameters $\theta$ and $\xi$ were omitted for simplicity, but the initial time $\sigma$ was considered together with the initial function as parameters in the equation. Combining the techniques of [9] and [16], and assuming the appropriate monotonicity condition (1.7), but without assuming the compatibility condition (1.3), the continuous differentiability of the parameter maps

$$
W^{1, \infty} \rightarrow \mathbb{R}^{n}, \quad \varphi \mapsto x(t, \sigma, \varphi)
$$

and

$$
W^{1, \infty} \rightarrow C, \quad \varphi \mapsto x_{t}(\cdot, \sigma, \varphi)
$$

were proved for a fixed $t$ and $\sigma$ in a neighborhood of a fixed initial function. Note that with this technique similar result can't be given using the $W^{1, \infty}$-norm on the state-space without using the compatibility condition.

Assuming the compatibility condition (1.3) it was also shown in [12] that the maps

$$
[0, \alpha) \rightarrow \mathbb{R}^{n}, \quad \sigma \mapsto x(t, \sigma, \varphi)
$$

and

$$
[0, \alpha) \rightarrow C, \quad \sigma \mapsto x_{t}(\cdot, \sigma, \varphi)
$$

are differentiable for all $t \in[\sigma-r, \alpha]$ and $t \in[\sigma, \alpha]$, respectively, and $\sigma, \varphi$ in a neighborhood of a fixed parameter $(\sigma, \varphi)$, and where $\alpha>0$ is a certain constant. Assuming that the functions $f$ and $\tau$ have a special form in (1.8), i.e., for equations of the form

$$
\begin{aligned}
\dot{x}(t)= & \bar{f}\left(t, x\left(t-\lambda^{1}(t)\right), \ldots, x\left(t-\lambda^{m}(t)\right), \int_{-r}^{0} A(t, \theta) x(s+\theta) d s,\right. \\
& \left.x\left(t-\bar{\tau}\left[t, x\left(t-\xi^{1}(t)\right), \ldots, x\left(t-\xi^{\ell}(t)\right), \int_{-r}^{0} B(t, \theta) x(s+\theta) d s\right]\right)\right)
\end{aligned}
$$


the differentiability of the map

$$
[0, \alpha) \rightarrow \mathbb{R}^{n}, \quad \sigma \mapsto x(t, \sigma, \varphi)
$$

was shown in [12] for $t \in[\sigma, \alpha]$ using the monotonicity assumption (1.7), but without the compatibility condition (1.3). Note that in this case similar result does not hold for the map $\sigma \mapsto x_{t}(\cdot, \sigma, \varphi)$ using the $C$-norm, which is not surprising, since it is easy to see [12] that the map $\sigma \mapsto x(t, \sigma, \varphi)$ is differentiable at the point $t=\sigma$ if and only if a compatibility condition similar to (1.3) is satisfied.

We refer the interested reader for related works on dependence of the solutions on parameters in SD-DDEs to [19, 20], and for similar works in neutral SD-DDEs to [11, 13, 23].

The organization of this paper is the following. In Section 2 we summarize some notations and preliminary results that will be used in the manuscript. In Section 3 first we list the detailed assumptions on the IVP (1.1)-(1.2) we will need in our differentiability results later, and formulate a well-posedness result (Theorem 3.1) concerning the IVP (1.1)-(1.2), and prove some estimates will be essential later.

In Section 4 using and extending the method introduced in [12], we discuss first order differentiability of the parameter maps associated to the IVP (1.1)-(1.2). In the main result of this section (see Theorem 4.7 below) we show the differentiability of the parameter maps (1.4) and (1.5) without using the compatibility condition (1.3), and also relaxing the monotonicity condition (1.7) to the condition that the time lag function $t \mapsto t-\tau\left(t, x_{t}, \xi\right)$ is "piecewise strictly monotone" in the sense of Definition 2.6. Note that omitting the compatibility condition is essential in the application of this results in [14], where we prove the convergence of the quasilinearization method in the problem of parameter estimation. Also, in this application the existence of the derivative is needed in this strong, pointwise sense, i.e., the differentiability of the map (1.4) is used in [14].

In Section 5 the main result is Theorem 5.17, which proves twice continuous differentiability of the maps

$$
W^{2, \infty} \times \Theta \times \Xi \rightarrow \mathbb{R}^{n}, \quad(\varphi, \theta, \xi) \mapsto x(t, \varphi, \theta, \xi)
$$

and

$$
W^{2, \infty} \times \Theta \times \Xi \rightarrow C, \quad(\varphi, \theta, \xi) \mapsto x_{t}(\cdot, \varphi, \theta, \xi)
$$

at a parameter value $(\varphi, \theta, \xi)$ satisfying the compatibility condition (1.3) and such that the corresponding time lag function $t \mapsto \tau\left(t, x_{t}, \xi\right)$ is piecewise strictly monotone in the sense of Definition 2.6. Under some additional condition, the continuity of the second derivative wrt the parameters is obtained in a certain sense. The only result known in the literature for the existence of a second derivative wrt the parameters in SD-DDEs is the result of Chen, $\mathrm{Hu}$ and $\mathrm{Wu}[2]$, where the second order differentiability is proved only using a weak $W^{1, p}$-norm on the state-space. Note that our result shows the existence of the second derivative in a pointwise sense, i.e., at each fixed $t$, moreover, the technique of the proof is simpler. 


\section{Notations and preliminaries}

Throughout the manuscript $r>0$ is a fixed constant and $x_{t}:[-r, 0] \rightarrow \mathbb{R}^{n}, x_{t}(\theta):=x(t+\theta)$ is the segment function. To avoid confusion with the notation of the segment function, sequences of functions are denoted using the upper index: $x^{k}$.

$\mathbb{N}$ and $\mathbb{N}_{0}$ denote the set of positive and nonnegative integers, respectively. A fixed norm on $\mathbb{R}^{n}$ and its induced matrix norm on $\mathbb{R}^{n \times n}$ are both denoted by $|\cdot|$. $C$ denotes the Banach space of continuous functions $\psi:[-r, 0] \rightarrow \mathbb{R}^{n}$ equipped with the norm $|\psi|_{C}=\max \{|\psi(\zeta)|:$ $\zeta \in[-r, 0]\} . C^{1}$ is the space of continuously differentiable functions $\psi:[-r, 0] \rightarrow \mathbb{R}^{n}$ where the norm is defined by $|\psi|_{C^{1}}=\max \left\{|\psi|_{C},|\dot{\psi}|_{C}\right\} . \quad L^{\infty}$ is the space of Lebesgue-measurable functions $\psi:[-r, 0] \rightarrow \mathbb{R}^{n}$ which are essentially bounded. The norm on $L^{\infty}$ is denoted by $|\psi|_{L^{\infty}}=\operatorname{ess} \sup \{|\psi(\zeta)|: \zeta \in[-r, 0]\}$. $W^{1, p}$ denotes the Banach-space of absolutely continuous functions $\psi:[-r, 0] \rightarrow \mathbb{R}^{n}$ of finite norm defined by

$$
|\psi|_{W^{1, p}}:=\left(\int_{-r}^{0}|\psi(\zeta)|^{p}+|\dot{\psi}(\zeta)|^{p} d \zeta\right)^{1 / p}, \quad 1 \leq p<\infty,
$$

and for $p=\infty$

$$
|\psi|_{W^{1, \infty}}:=\max \left\{|\psi|_{C},|\dot{\psi}|_{L^{\infty}}\right\} .
$$

We note that $W^{1, \infty}$ is equal to the space of Lipschitz continuous functions from $[-r, 0]$ to $\mathbb{R}^{n}$. The subset of $W^{1, \infty}$ consisting of those functions which have absolutely continuous first derivative and essentially bounded second derivative is denoted by $W^{2, \infty}$, where the norm is defined by

$$
|\psi|_{W^{2, \infty}}:=\max \left\{|\psi|_{C},|\dot{\psi}|_{C},|\ddot{\psi}|_{L^{\infty}}\right\} .
$$

If the domain or the range of the functions is different from $[-r, 0]$ and $\mathbb{R}^{n}$, respectively, we will use a more detailed notation. E.g., $C(X, Y)$ denotes the space of continuous functions mapping from $X$ to $Y$. Finally, $\mathcal{L}(X, Y)$ denotes the space of bounded linear operators from $X$ to $Y$, where $X$ and $Y$ are normed linear spaces. An open ball in the normed linear space $X$ centered at a point $x \in X$ with radius $\delta$ is denoted by $\mathcal{B}_{X}(x ; \delta):=\{y \in Y:|x-y|<\delta\}$.

The derivative of a single variable function $v(t)$ wrt $t$ is denoted by $\dot{v}$. Note that all derivatives we use in this paper are Fréchet derivatives. The partial derivatives of a function $g: X_{1} \times X_{2} \rightarrow Y$ wrt the first and second variables will be denoted by $D_{1} g$ and $D_{2} g$, respectively. The secondorder partial derivative wrt its $i$ th and $j$ th variables $(i, j=1,2)$ of the function $g: X_{1} \times X_{2} \rightarrow Y$ at the point $\left(x_{1}, x_{2}\right) \in X_{1} \times X_{2}$ is the bounded bilinear operator $A\langle\cdot, \cdot\rangle: X_{i} \times X_{j} \rightarrow Y$, if

$$
\lim _{k \rightarrow 0} \sup _{h \neq 0} \frac{\left|D_{i} g\left(x_{1}+k \delta_{1 j}, x_{2}+k \delta_{2 j}\right) h-D_{i} g\left(x_{1}, x_{2}\right) h-A\langle h, k\rangle\right|_{Y}}{|h|_{X_{i}}|k|_{X_{1}}}=0, \quad h \in X_{i}, k \in X_{j},
$$

where $\delta_{i j}=1$ for $i=j$ and $\delta_{i j}=0$ for $i \neq j$ is the Kronecker-delta. We will use the notation $D_{i j} g\left(x_{1}, x_{2}\right)=A$. The norm of the bilinear operator $A\langle\cdot, \cdot\rangle: X_{i} \times X_{j} \rightarrow Y$ is defined by

$$
|A|_{\mathcal{L}^{2}\left(X_{i} \times X_{j}, Y\right)}:=\sup \left\{\frac{|A\langle h, k\rangle|_{Y}}{|h|_{X_{i}}|k|_{X_{j}}}: h \in X_{i}, h \neq 0, k \in X_{j}, k \neq 0\right\} .
$$

In the case when $X_{1}=\mathbb{R}$, we simply write $D_{1} g\left(x_{1}, x_{2}\right)$ instead of the more precise notation $D_{1} g\left(x_{1}, x_{2}\right)$ 1, i.e., here $D_{1} g$ denotes the value in $Y$ instead of the linear operator $\mathcal{L}(\mathbb{R}, Y)$. In the 
case when, let say, $X_{2}=\mathbb{R}^{n}=Y$, then we identify the linear operator $D_{2} g\left(x_{1}, x_{2}\right) \in \mathcal{L}\left(\mathbb{R}^{n}, \mathbb{R}^{n}\right)$ by an $n \times n$ matrix.

Next we formulate a result which is a simple consequence of the Gronwall's lemma.

Lemma 2.1 (see, e.g., [12]) Suppose $a>0, b:[0, \alpha] \rightarrow[0, \infty)$ and $u:[-r, \alpha] \rightarrow \mathbb{R}^{n}$ are continuous functions such that $a \geq\left|u_{0}\right|_{C}$, and

$$
|u(t)| \leq a+\int_{0}^{t} b(s)\left|u_{s}\right|_{C} d s, \quad t \in[0, \alpha]
$$

Then

$$
|u(t)| \leq\left|u_{t}\right|_{C} \leq a e^{\int_{0}^{\alpha} b(s) d s}, \quad t \in[0, \alpha]
$$

Lemma 2.2 Suppose $\psi \in W^{1, \infty}$. Then

$$
|\psi(b)-\psi(a)| \leq|\dot{\psi}|_{L^{\infty}}|b-a|
$$

for every $[a, b] \subset[-r, 0]$.

We recall the following result from [1], which was essential to prove differentiability wrt parameters in SD-DDEs in [2], [12] and [16]. We state the result in a simplified form we need later, it is formulated in a more general form in [1]. Note that the second part of the lemma was stated in [1] under the assumption $\left|u^{k}-u\right|_{W^{1, \infty}([0, \alpha], \mathbb{R})} \rightarrow 0$ as $k \rightarrow \infty$, but this stronger assumption on the convergence is not needed in the proof. See also the proof of Lemma 4.26 in [8].

Lemma 2.3 ([1]) Let $g \in L^{1}\left([c, d], \mathbb{R}^{n}\right), \varepsilon>0$, and $u \in \mathcal{A}(\varepsilon)$, where

$$
\mathcal{A}(\varepsilon):=\left\{v \in W^{1, \infty}([a, b],[c, d]): \dot{v}(s) \geq \varepsilon \text { for a.e. } s \in[a, b]\right\} .
$$

Then

$$
\int_{a}^{b}|g(u(s))| d s \leq \frac{1}{\varepsilon} \int_{c}^{d}|g(s)| d s .
$$

Moreover, if the sequence $u^{k} \in \mathcal{A}(\varepsilon)$ is such that $\left|u^{k}-u\right|_{C([a, b], \mathbb{R})} \rightarrow 0$ as $k \rightarrow \infty$, then

$$
\lim _{k \rightarrow \infty} \int_{a}^{b}\left|g\left(u^{k}(s)\right)-g(u(s))\right| d s=0 .
$$

Remark 2.4 Changing to the new variable $s=-t$ in the integrals in (2.3) and (2.4) give easily that the statements of Lemma 2.3 hold also in the case when conditions $u, u^{k} \in \mathcal{A}(\varepsilon)$ are replaced by $-u,-u^{k} \in \mathcal{A}(\varepsilon)$.

In the next lemma we relax the condition $u \in \mathcal{A}(\varepsilon)$ of the previous lemma. 
Lemma 2.5 Suppose $g \in L^{\infty}([c, d], \mathbb{R})$, and $u:[a, b] \rightarrow[c, d]$ is an absolutely continuous function, and

$$
\operatorname{essinf}\left\{\dot{u}(s): s \in\left[a^{\prime}, b^{\prime}\right]\right\}>0, \quad \text { for all }\left[a^{\prime}, b^{\prime}\right] \subset(a, b)
$$

Then the composite function $g \circ u \in L^{\infty}([a, b], \mathbb{R})$, and $|g \circ u|_{L^{\infty}([a, b], \mathbb{R})} \leq|g|_{L^{\infty}([c, d], \mathbb{R})}$.

Proof First note that since $u$ is absolutely continuous, it is a.e. differentiable on $[a, b]$, and condition (2.5) yields that $u$ is strictly monotone increasing on $[a, b]$. Let $G:=\{v \in[c, d]$ : $g(v)$ is not defined or $\left.|g(v)|>|g|_{L^{\infty}([c, d], \mathbb{R})}\right\}$. Then meas $(G)=0$. Let $A:=\{t \in[a, b]$ : $g(u(t))$ is not defined or $\left.|g(u(t))|>|g|_{L^{\infty}([c, d], \mathbb{R})}\right\}$. Clearly, $A=u^{-1}(G)$. Let $0<\varepsilon<(b-a) / 2$ be fixed. Then let $c^{\prime}:=u(a+\varepsilon), d^{\prime}:=u(b-\varepsilon)$, and let $M:=\operatorname{ess} \inf \{\dot{u}(s): s \in[a+\varepsilon, b-\varepsilon]\}$. Then (2.5) yields $M>0$. Since $G$ is of measure 0 , there exist open intervals $\left(c_{i}, d_{i}\right), i \in \mathbb{N}$ such that

$$
G \subset \bigcup_{i=1}^{\infty}\left(c_{i}, d_{i}\right) \text { and } \sum_{i=1}^{\infty}\left(d_{i}-c_{i}\right)<\varepsilon M .
$$

We have

$$
A=u^{-1}(G)=u^{-1}\left(G \cap\left[c, c^{\prime}\right]\right) \cup u^{-1}\left(G \cap\left[c^{\prime}, d^{\prime}\right]\right) \cup u^{-1}\left(G \cap\left[d^{\prime}, d\right]\right),
$$

and the monotonicity of $u$ yields $u^{-1}\left(G \cap\left[c, c^{\prime}\right]\right) \subset[a, a+\varepsilon], u^{-1}\left(G \cap\left[d^{\prime}, d\right]\right) \subset[b-\varepsilon, b]$, and

$$
u^{-1}\left(G \cap\left[c^{\prime}, d^{\prime}\right]\right) \subset u^{-1}\left(\left[c^{\prime}, d^{\prime}\right] \cap \bigcup_{i=1}^{\infty}\left[c_{i}, d_{i}\right]\right)=\bigcup_{i=1}^{\infty} u^{-1}\left(\left[c^{\prime}, d^{\prime}\right] \cap\left[c_{i}, d_{i}\right]\right)=\bigcup_{i=1}^{\infty}\left[a_{i}, b_{i}\right]
$$

where $a_{i}:=u^{-1}\left(\max \left\{c^{\prime}, c_{i}\right\}\right)$ and $b_{i}:=u^{-1}\left(\min \left\{d^{\prime}, d_{i}\right\}\right)$. The definition of $M$ yields

$$
d_{i}-c_{i} \geq \min \left\{d^{\prime}, d_{i}\right\}-\max \left\{c^{\prime}, c_{i}\right\}=u\left(b_{i}\right)-u\left(a_{i}\right)=\int_{a_{i}}^{b_{i}} \dot{u}(s) d s \geq M\left(b_{i}-a_{i}\right) .
$$

Therefore $A \subset[a, a+\varepsilon] \cup[b-\varepsilon, b] \cup \bigcup_{i=1}^{\infty}\left[a_{i}, b_{i}\right]$, and the sum of the length of the closed intervals covering $A$ is less than $3 \varepsilon$. Since $\varepsilon>0$ is arbitrary, we get that $A$ is Lebesgue-measurable and $\operatorname{meas}(A)=0$.

We show that $g \circ u$ is Lebesgue-measurable. Let $\kappa \in \mathbb{R}$, and define $G_{\kappa}:=\{v \in[c, d]$ : $g(v)$ is defined and $g(v)<\kappa\} . \quad G_{\kappa}$ is a Lebesgue-measurable set, since $g \in L^{\infty}([c, d], \mathbb{R})$. Therefore there exists a closed set $F_{\kappa}$ such that $F_{\kappa} \subset G_{\kappa}$ and meas $\left(G_{\kappa} \backslash F_{\kappa}\right)=0$. Since $u$ is continuous, $u^{-1}\left(F_{\kappa}\right)$ is a closed set, and therefore, it is Lebesgue-measurable. Moreover, $u^{-1}\left(G_{\kappa}\right)=u^{-1}\left(F_{\kappa}\right) \cup u^{-1}\left(G_{\kappa} \backslash F_{\kappa}\right)$, and as in the first part of the proof, we get that $u^{-1}\left(G_{\kappa} \backslash F_{\kappa}\right)$ is measurable, and so is $u^{-1}\left(G_{\kappa}\right)$.

Clearly, the statement of the previous Lemma is also valid if (2.5) is changed to

$$
\operatorname{ess} \sup \left\{\dot{u}(s): s \in\left[a^{\prime}, b^{\prime}\right]\right\}<0, \quad \text { for all }\left[a^{\prime}, b^{\prime}\right] \subset(a, b) \text {. }
$$

We will use the following notation. 
Definition 2.6 $\mathcal{P M}([a, b],[c, d])$ denotes the set of absolutely continuous functions $u:[a, b] \rightarrow$ $[c, d]$ which are piecewise strictly monotone on $[a, b]$ in the sense that there exists a finite mesh $a=t_{0}<t_{1}<\cdots<t_{m-1}<t_{m}=b$ of $[a, b]$ such that for all $i=0,1, \ldots, m-1$ either

$$
\operatorname{ess} \inf \left\{\dot{u}(s): s \in\left[a^{\prime}, b^{\prime}\right]\right\}>0, \quad \text { for all }\left[a^{\prime}, b^{\prime}\right] \subset\left(t_{i}, t_{i+1}\right)
$$

or

$$
\operatorname{ess} \sup \left\{\dot{u}(s): s \in\left[a^{\prime}, b^{\prime}\right]\right\}<0, \quad \text { for all }\left[a^{\prime}, b^{\prime}\right] \subset\left(t_{i}, t_{i+1}\right) \text {. }
$$

Lemma 2.5 implies the next result immediately.

Lemma 2.7 Suppose $g \in L^{\infty}\left([c, d], \mathbb{R}^{n}\right)$, and $u \in \mathcal{P} \mathcal{M}([a, b],[c, d])$. Then the composite function $g \circ u \in L^{\infty}\left([a, b], \mathbb{R}^{n}\right)$ and $|g \circ u|_{L^{\infty}\left([a, b], \mathbb{R}^{n}\right)} \leq|g|_{L^{\infty}\left([c, d], \mathbb{R}^{n}\right)}$.

The next lemma generalizes the convergence property (2.4) to the class $\mathcal{P} \mathcal{M}$. We comment that to prove the convergence property (2.4) for $u, u^{k} \in \mathcal{P} \mathcal{M}([a, b],[c, d])$, we need the stronger assumption $\left|u^{k}-u\right|_{W^{1, \infty}([a, b], \mathbb{R})} \rightarrow 0$ instead of $\left|u^{k}-u\right|_{C([a, b], \mathbb{R})} \rightarrow 0$ what is used in Lemma 2.3.

Lemma 2.8 Suppose $g \in L^{\infty}\left([c, d], \mathbb{R}^{n}\right)$, and $u, u^{k} \in \mathcal{P} \mathcal{M}([a, b],[c, d]) \quad(k \in \mathbb{N})$ satisfying

$$
\left|u^{k}-u\right|_{W^{1, \infty}([a, b], \mathbb{R})} \rightarrow 0, \quad \text { as } k \rightarrow \infty .
$$

Then

$$
\int_{a}^{b}\left|g\left(u^{k}(s)\right)-g(u(s))\right| d s \rightarrow 0, \quad \text { as } k \rightarrow \infty .
$$

Proof Clearly, it is enough to show (2.7) for the case when $g$ is real valued, i.e., $n=1$.

First note that Lemma 2.7 yields $g \circ u, g \circ u^{k} \in L^{\infty}([a, b], \mathbb{R})$. We prove (2.7) in three steps.

(i) First suppose that $g \in L^{\infty}([c, d], \mathbb{R})$ is the characteristic function of an interval $[e, f] \subset$ $[c, d]$, i.e., $g=\chi_{[e, f]}$. Then $\left|\chi_{[e, f]}\left(u^{k}(s)\right)-\chi_{[e, f]}(u(s))\right|$ is either 0 or 1 , hence

$$
\operatorname{meas}\left(\left\{s \in[a, b]: \chi_{[e, f]}\left(u^{k}(s)\right) \neq \chi_{[e, f]}(u(s))\right\}\right) \leq 4\left|u^{k}-u\right|_{C([a, b], \mathbb{R})},
$$

and so

$$
\int_{a}^{b}\left|\chi_{[e, f]}\left(u^{k}(s)\right)-\chi_{[e, f]}(u(s))\right| d s \leq 4\left|u^{k}-u\right|_{C([a, b], \mathbb{R})} \rightarrow 0, \quad \text { as } k \rightarrow \infty .
$$

(ii) Suppose $g$ is a step function, i.e., $g=\sum_{i=1}^{m} c_{i} \chi_{A_{i}}$, where $A_{i}$ are pairwise disjoint intervals with $\cup_{i=1}^{m} A_{i}=[c, d]$. Then

$$
\int_{a}^{b}\left|g\left(u^{k}(s)\right)-g(u(s))\right| d s \leq \sum_{i=1}^{m}\left|c_{i}\right| 4\left|u^{k}-u\right|_{C([a, b], \mathbb{R})} \rightarrow 0, \quad \text { as } k \rightarrow \infty .
$$

(iii) Let $a=t_{0}<t_{1}<\cdots<t_{m}=b$ be the mesh points of $u$ from the Definition 2.6, and let $0<\varepsilon<\min \left\{t_{i+1}-t_{i}: i=0, \ldots, m-1\right\} / 2$ be fixed, and introduce $t_{i}^{\prime}:=t_{i}+\varepsilon$ for $i=0, \ldots, m-1$ and $t_{i}^{\prime \prime}:=t_{i}-\varepsilon$ for $i=1, \ldots, m, t_{0}^{\prime \prime}:=a, t_{m}^{\prime}:=b$, and let

$$
M:=\min _{i=0, \ldots, m-1} \underset{t \in\left[t_{i}^{\prime}, t_{i+1}^{\prime \prime}\right]}{\operatorname{essinf}}|\dot{u}(t)| .
$$


We have $M>0$, since $u \in \mathcal{P} \mathcal{M}([a, b],[c, d])$.

The set of step functions is dense in $L^{1}([c, d], \mathbb{R})$ (see, e.g., [4]), so for a fixed $g \in L^{\infty}([c, d], \mathbb{R}$ ) and $0<\delta<\varepsilon M / m$ there exists a step function $h:[c, d] \rightarrow \mathbb{R}$ such that $|g-h|_{L^{1}([c, d], \mathbb{R})}<\delta$. Let $h=\sum_{i=1}^{m} c_{i} \chi_{A_{i}}$, where $A_{i}$ are pairwise disjoint intervals with $\cup_{i=1}^{m} A_{i}=[c, d]$, and define $h^{*}:=\sum_{i=1}^{m} c_{i}^{*} \chi_{A_{i}}$, where

$$
c_{i}^{*}:= \begin{cases}c_{i}, & \text { if }\left|c_{i}\right| \leq|g|_{L^{\infty}([c, d], \mathbb{R})}+1, \\ |g|_{L^{\infty}([c, d], \mathbb{R})}, & \text { if } c_{i}>|g|_{L^{\infty}([c, d], \mathbb{R})}+1, \\ -|g|_{L^{\infty}([c, d], \mathbb{R})}, & \text { if } c_{i}<-|g|_{L^{\infty}([c, d], \mathbb{R})}-1 .\end{cases}
$$

Then it is easy to check that $\left|g(v)-h^{*}(v)\right| \leq 1$ for a.e. $v \in[c, d]$, and

$$
\int_{c}^{d}\left|g(v)-h^{*}(v)\right| d v \leq \int_{c}^{d}|g(v)-h(v)| d v<\delta .
$$

We have therefore

$$
\begin{aligned}
\int_{a}^{b} \mid g(u(s)) & -h^{*}(u(s)) \mid d s \\
& =\sum_{i=0}^{m} \int_{t_{i}^{\prime \prime}}^{t_{i}^{\prime}}\left|g(u(s))-h^{*}(u(s))\right| d s+\sum_{i=0}^{m-1} \int_{t_{i}^{\prime}}^{t_{i+1}^{\prime \prime}}\left|g(u(s))-h^{*}(u(s))\right| d s \\
\leq & 2 \varepsilon(m+1)+\sum_{i=0}^{m-1} \int_{t_{i}^{\prime}}^{t_{i+1}^{\prime \prime}}\left|g(u(s))-h^{*}(u(s))\right| \dot{u}(s) \frac{1}{\dot{u}(s)} d s \\
\leq & 2 \varepsilon(m+1)+\frac{1}{M} \sum_{i=0}^{m-1}\left|\int_{u\left(t_{i}^{\prime}\right)}^{u\left(t_{i+1}^{\prime \prime}\right)}\right| g(v)-h^{*}(v)|d v| \\
\leq & 2 \varepsilon(m+1)+\frac{\delta m}{M} \\
\leq & (2 m+3) \varepsilon .
\end{aligned}
$$

Assumption (2.6) yields that there exist $k_{0}>0$ such that $\left|u^{k}-u\right|_{W^{1, \infty}([a, b], \mathbb{R})}<\frac{M}{2}$ for $k \geq k_{0}$. Then for $k \geq k_{0}$ it follows $\left|\dot{u}^{k}(s)\right| \geq \frac{M}{2}$ for a.e. $s \in\left[t_{i}^{\prime}, t_{i+1}^{\prime \prime}\right]$ and $i=0, \ldots, m-1$. Therefore similarly to the previous estimate we have for $k \geq k_{0}$

$$
\int_{a}^{b}\left|g\left(u^{k}(s)\right)-h^{*}\left(u^{k}(s)\right)\right| d s \leq 2 \varepsilon(m+1)+\frac{2 \delta m}{M} \leq(2 m+4) \varepsilon .
$$

Using the above inequalities we get

$$
\begin{aligned}
\int_{a}^{b}\left|g\left(u^{k}(s)\right)-g(u(s))\right| d s & \\
\leq & \int_{a}^{b}\left|g\left(u^{k}(s)\right)-h^{*}\left(u^{k}(s)\right)\right| d s+\int_{a}^{b}\left|h^{*}\left(u^{k}(s)\right)-h^{*}(u(s))\right| d s \\
& +\int_{a}^{b}\left|g(u(s))-h^{*}(u(s))\right| d s \\
\leq & (4 m+7) \varepsilon+\int_{a}^{b}\left|h^{*}\left(u^{k}(s)\right)-h^{*}(u(s))\right| d s, \quad k \geq k_{0},
\end{aligned}
$$

which yields (2.7) using part (ii), since $\varepsilon>0$ is arbitrary close to 0 . 
Lemma 2.9 Suppose $f^{k, h} \in L^{\infty}\left([c, d], \mathbb{R}^{n}\right)$ for $k \in \mathbb{N}$ and $h \in H$ for some fixed parameter set $H$,

$$
\lim _{k \rightarrow \infty} \sup _{h \in H} \int_{c}^{d}\left|f^{k, h}(s)\right| d s=0,
$$

and there exists $A \geq 0$ such that $\left|f^{k, h}(s)\right| \leq A$ for $k \in \mathbb{N}, h \in H$ and a.e. $s \in[c, d]$. Let $u, u^{k} \in \mathcal{P} \mathcal{M}([a, b],[c, d])(k \in \mathbb{N})$ be such that $(2.6)$ holds. Then

$$
\lim _{k \rightarrow \infty} \sup _{h \in H} \int_{a}^{b}\left|f^{k, h}\left(u^{k}(s)\right)\right| d s=0
$$

Proof Let $a=t_{0}<t_{1}<\cdots<t_{m}=b$ be the mesh points of $u$ from the Definition 2.6, and let $0<\varepsilon<\min \left\{t_{i+1}-t_{i}: i=0, \ldots, m-1\right\} / 2$ be fixed, let $t_{i}^{\prime}$ and $t_{i}^{\prime \prime}$ be defined as in the proof of Lemma 2.8, and let $M$ be defined by (2.8). Let $k_{0}$ be such that $\left|u^{k}-u\right|_{W^{1, \infty}([a, b], \mathbb{R})} \leq M / 2$ for $k \geq k_{0}$. Then for $k \geq k_{0}$ it follows $\left|\dot{u}^{k}(s)\right| \geq \frac{M}{2}$ for a.e. $s \in\left[t_{i}^{\prime}, t_{i+1}^{\prime \prime}\right]$ and $i=0, \ldots, m-1$. Since $u^{k} \in \mathcal{P} \mathcal{M}([a, b],[c, d])$, it follows from Lemma 2.7 that $\left|f^{k, h}\left(u^{k}(s)\right)\right| \leq A$ for $k \in \mathbb{N}, h \in H$ and a.e. $s \in[a, b]$. Therefore for any $k \in \mathbb{N}$ and $h \in H$ we have

$$
\begin{aligned}
\int_{a}^{b}\left|f^{k, h}\left(u^{k}(s)\right)\right| d s & =\sum_{i=0}^{m} \int_{t_{i}^{\prime \prime}}^{t_{i}^{\prime}}\left|f^{k, h}\left(u^{k}(s)\right)\right| d s+\sum_{i=0}^{m-1} \int_{t_{i}^{\prime}}^{t_{i+1}^{\prime \prime}}\left|f^{k, h}\left(u^{k}(s)\right)\right| d s \\
& \leq(m+1) A 2 \varepsilon+\frac{2 m}{M} \int_{c}^{d}\left|f^{k, h}(s)\right| d s .
\end{aligned}
$$

Then

$$
\sup _{h \in H} \int_{a}^{b}\left|f^{k, h}\left(u^{k}(s)\right)\right| d s \leq(m+1) A 2 \varepsilon+\sup _{h \in H} \frac{2 m}{M} \int_{c}^{d}\left|f^{k, h}(s)\right| d s,
$$

which proves the statement, since $\varepsilon$ is arbitrarily close to 0 .

\section{Well-posedness and continuous dependence on parameters}

In this section we list all the assumptions we need later on the IVP (1.1)-(1.2), and show some basic results including the well-posedness of the IVP and Lipschitz continuous dependence of the solutions on the parameters $\varphi, \theta$ and $\gamma$.

Suppose $\Omega_{1} \subset C, \Omega_{2} \subset \mathbb{R}^{n}, \Omega_{3} \subset \Theta, \Omega_{4} \subset \Xi$ are open subsets of the respective spaces. $T>0$ is finite or $T=\infty$, in which case $[0, T]$ denotes the interval $[0, \infty)$.

We assume

(i) $f: \mathbb{R} \times C \times \mathbb{R}^{n} \times \Theta \supset[0, T] \times \Omega_{1} \times \Omega_{2} \times \Omega_{3} \rightarrow \mathbb{R}^{n}$ is continuous;

(ii) $f(t, \psi, u, \theta)$ is locally Lipschitz continuous in $\psi, u$ and $\theta$, i.e., for every finite $\alpha \in(0, T]$, for every closed subset $M_{1} \subset \Omega_{1}$ of $C$ which is also a bounded subset of $W^{1, \infty}$, compact subset $M_{2} \subset \Omega_{2}$ of $\mathbb{R}^{n}$, and closed and bounded subset $M_{3} \subset \Omega_{3}$ of $\Theta$ there exists a constant $L_{1}=L_{1}\left(\alpha, M_{1}, M_{2}, M_{3}\right)$ such that

$$
|f(t, \psi, u, \theta)-f(t, \bar{\psi}, \bar{u}, \bar{\theta})| \leq L_{1}\left(|\psi-\bar{\psi}|_{C}+|u-\bar{u}|+|\theta-\bar{\theta}|_{\Theta}\right),
$$

for $t \in[0, \alpha], \psi, \bar{\psi} \in M_{1}, u, \bar{u} \in M_{2}$ and $\theta, \bar{\theta} \in M_{3}$; 
(iii) $f: \mathbb{R} \times C \times \mathbb{R}^{n} \times \Theta \supset[0, T] \times \Omega_{1} \times \Omega_{2} \times \Omega_{3} \rightarrow \mathbb{R}^{n}$ is continuously differentiable wrt its second, third and fourth arguments;

(iv) $f(t, \psi, u, \theta)$ is locally Lipschitz continuous wrt $t$, i.e., for every finite $\alpha \in(0, T]$, for every closed subset $M_{1} \subset \Omega_{1}$ of $C$ which is also a bounded subset of $W^{1, \infty}$, compact subset $M_{2} \subset \Omega_{2}$ of $\mathbb{R}^{n}$, and closed and bounded subset $M_{3} \subset \Omega_{3}$ of $\Theta$ there exists a constant $L_{1}=L_{1}\left(\alpha, M_{1}, M_{2}, M_{3}\right)$ such that

$$
|f(t, \psi, u, \theta)-f(\bar{t}, \psi, u, \theta)| \leq L_{1}|t-\bar{t}|
$$

for $t, \bar{t} \in[0, \alpha], \psi \in M_{1}, u \in M_{2}$ and $\theta \in M_{3}$;

(v) $D_{2} f, D_{3} f$ and $D_{4} f$ are locally Lipschitz continuous wrt all of their arguments, i.e., for every finite $\alpha \in(0, T]$, for every closed subset $M_{1} \subset \Omega_{1}$ of $C$ which is also a bounded subset of $W^{1, \infty}$, compact subset $M_{2} \subset \Omega_{2}$ of $\mathbb{R}^{n}$, and closed and bounded subset $M_{3} \subset \Omega_{3}$ of $\Theta$ there exists $L_{3}=L_{3}\left(\alpha, M_{1}, M_{2}, M_{3}\right)$ such that

$$
\left|D_{i} f(t, \psi, u, \theta)-D_{i} f(\bar{t}, \bar{\psi}, \bar{u}, \bar{\theta})\right|_{\mathcal{L}\left(Y_{i}, \mathbb{R}^{n}\right)} \leq L_{3}\left(|t-\bar{t}|+|\psi-\bar{\psi}|_{C}+|u-\bar{u}|+|\theta-\bar{\theta}|_{\Theta}\right)
$$

for $i=2,3,4, t, \bar{t} \in[0, \alpha], \psi, \bar{\psi} \in M_{1}, u, \bar{u} \in M_{2}$ and $\theta, \bar{\theta} \in M_{3}$, where $Y_{2}:=C$, $Y_{3}:=\mathbb{R}^{n}$ and $Y_{4}:=\Theta$

(vi) $D_{2} f, D_{3} f$ and $D_{4} f$ are continuously differentiable wrt their second, third and fourth arguments on $[0, T] \times \Omega_{1} \times \Omega_{2} \times \Omega_{3}$;

(i) $\tau: \mathbb{R} \times C \times \Xi \supset[0, T] \times \Omega_{1} \times \Omega_{4} \rightarrow[0, r] \subset \mathbb{R}$ is continuous;

(ii) $\tau(t, \psi, \xi)$ is locally Lipschitz continuous in $\psi$ and $\xi$ in the following sense: for every finite $\alpha \in(0, T]$, closed subset $M_{1} \subset \Omega_{1}$ of $C$ which is also a bounded subset of $W^{1, \infty}$, and closed and bounded subset $M_{4} \subset \Omega_{4}$ of $\Xi$ there exists a constant $L_{2}=$ $L_{2}\left(\alpha, M_{1}, M_{4}\right)$ such that

$$
|\tau(t, \psi, \xi)-\tau(t, \bar{\psi}, \bar{\xi})| \leq L_{2}\left(|\psi-\bar{\psi}|_{C}+|\xi-\bar{\xi}|_{\Xi}\right)
$$

for $t \in[0, \alpha], \psi, \bar{\psi} \in M_{1}, \xi, \bar{\xi} \in M_{4}$;

(iii) $\tau:[0, T] \times C \times \Xi \supset[0, T] \times \Omega_{1} \times \Omega_{4} \rightarrow \mathbb{R}$ is continuously differentiable wrt its second and third arguments;

(iv) $\tau(t, \psi, \xi)$ is locally Lipschitz continuous in $t$, i.e., for every finite $\alpha \in(0, T]$, closed subset $M_{1} \subset \Omega_{1}$ of $C$ which is also a bounded subset of $W^{1, \infty}$, and closed and bounded subset $M_{4} \subset \Omega_{4}$ of $\Xi$ there exists a constant $L_{2}=L_{2}\left(\alpha, M_{1}, M_{4}\right)$ such that

$$
|\tau(t, \psi, \xi)-\tau(\bar{t}, \psi, \xi)| \leq L_{2}|t-\bar{t}|
$$

for $t, \bar{t} \in[0, \alpha], \psi \in M_{1}, \xi \in M_{4}$;

(v) for every finite $\alpha \in(0, T]$, closed subset $M_{1} \subset \Omega_{1}$ of $C$ which is also a bounded subset of $W^{1, \infty}$, and closed and bounded subset $M_{4} \subset \Omega_{4}$ of $\Xi$ there exists $L_{4}=$ $L_{4}\left(\alpha, M_{1}, M_{4}\right) \geq 0$ such that

$$
\left|\frac{d}{d t} \tau\left(t, y_{t}, \xi\right)-\frac{d}{d t} \tau\left(t, \bar{y}_{t}, \bar{\xi}\right)\right| \leq L_{4}\left(\left|y_{t}-\bar{y}_{t}\right|_{W^{1, \infty}}+|\xi-\bar{\xi}| \Xi\right), \quad \text { a.e. } t \in[0, \alpha],
$$

where $\xi, \bar{\xi} \in M_{4}$, and $y, \bar{y} \in W^{1, \infty}\left([-r, \alpha], \mathbb{R}^{n}\right)$ are such that $y_{t}, \bar{y}_{t} \in M_{1}$ for $t \in[0, \alpha]$; 
(vi) $D_{2} \tau$ and $D_{3} \tau$ are locally Lipschitz continuous wrt all arguments, i.e., for every finite $\alpha \in(0, T]$, closed subset $M_{1} \subset \Omega_{1}$ of $C$ which is also a bounded subset of $W^{1, \infty}$, and closed and bounded subset $M_{4} \subset \Omega_{4}$ of $\Xi$ there exists a constant $L_{5}=L_{5}\left(\alpha, M_{1}, M_{4}\right)$ such that

$$
\left|D_{i} \tau(t, \psi, \xi)-D_{i} \tau(\bar{t}, \bar{\psi}, \bar{\xi})\right|_{\mathcal{L}\left(Z_{i}, \mathbb{R}\right)} \leq L_{5}\left(|t-\bar{t}|+|\psi-\bar{\psi}|_{C}+|\xi-\bar{\xi}|_{\Xi}\right)
$$

for $i=2,3, t, \bar{t} \in[0, \alpha], \psi, \bar{\psi} \in M_{1}, \xi, \bar{\xi} \in M_{4}$, where $Z_{2}:=C$ and $Z_{3}:=\Xi ;$

(vii) $D_{2} \tau$ and $D_{3} \tau$ are continuously differentiable wrt their second and third arguments on $[0, T] \times \Omega_{1} \times \Omega_{4}$;

(viii) for every finite $\alpha \in(0, T]$, for every closed subset $M_{1} \subset \Omega_{1}$ of $C$ which is also a bounded subset of $W^{1, \infty}$, compact subset $M_{2} \subset \Omega_{2}$ of $\mathbb{R}^{n}$, and closed and bounded subsets $M_{3} \subset \Omega_{3}$ of $\Theta$ and $M_{4} \subset \Omega_{4}$ of $\Xi$ there exists $L_{6}=L_{6}\left(\alpha, M_{1}, M_{2}, M_{3}, M_{4}\right)$ such that

$$
\begin{aligned}
& \left|\frac{d}{d t} f\left(t, y_{t}, y\left(t-\tau\left(t, y_{t}, \xi\right)\right), \theta\right)-\frac{d}{d t} f\left(t, \bar{y}_{t}, \bar{y}\left(t-\tau\left(t, \bar{y}_{t}, \bar{\xi}\right)\right), \bar{\theta}\right)\right| \\
& \leq L_{6}\left(\left|y_{t}-\bar{y}_{t}\right|_{W^{1, \infty}}+|\xi-\bar{\xi}|_{\Xi}+|\theta-\bar{\theta}|_{\Xi}\right), \quad \text { a.e. } t \in[0, \alpha],
\end{aligned}
$$

where $\theta, \bar{\theta} \in M_{3}, \xi, \bar{\xi} \in M_{4}$, and $y, \bar{y} \in W^{1, \infty}\left([-r, \alpha], \mathbb{R}^{n}\right)$ are such that $y_{t}, \bar{y}_{t} \in M_{1}$ for $t \in[0, \alpha]$.

We introduce the parameter space

$$
\Gamma:=W^{1, \infty} \times \Theta \times \Xi
$$

equipped with the product norm $|\gamma|_{\Gamma}:=|\varphi|_{W^{1, \infty}}+|\theta|_{\Theta}+|\xi|_{\Xi}$ for $\gamma=(\varphi, \theta, \xi) \in \Gamma$, and the set of admissible parameters

$$
\Pi:=\left\{(\varphi, \theta, \xi) \in \Gamma: \varphi \in \Omega_{1}, \varphi(-\tau(0, \varphi)) \in \Omega_{2}, \theta \in \Omega_{3}, \xi \in \Omega_{4}\right\} .
$$

The next theorem shows that every admissible parameter $(\hat{\varphi}, \hat{\theta}, \hat{\xi}) \in \Pi$ has a neighborhood $P$ and there exists a constant $\alpha>0$ such that the IVP (1.1)-(1.2) has a unique solution on $[-r, \alpha]$ corresponding to all parameters $\gamma=(\varphi, \theta, \xi) \in P$. This solution will be denoted by $x(t, \gamma)$, and its segment function at $t$ is denoted by $x_{t}(\cdot, \gamma)$.

The well-posedness of several classes of SD-DDEs was studied in many papers (see, e.g., $[5,15,16,19,21,22]$. The next result is a variant of a result from [12] where the initial time is also considered as a parameter, but the parameters $\theta$ and $\xi$ were missing in the equation. The proof is similar to that of Theorem 3.1 in [12], (see also the analogous proof of Theorem 3.2 of the neutral case in [13]), therefore it is omitted here. The notations and estimates introduced in the next theorem will be essential in the following sections.

Theorem 3.1 Assume (A1) (i), (ii), (A2) (i), (ii), and let $\hat{\gamma} \in \Pi$. Then there exist $\delta>0$ and $0<\alpha \leq T$ finite numbers such that

(i) for all $\gamma=(\varphi, \theta, \xi) \in P:=\mathcal{B}_{\Gamma}(\hat{\gamma} ; \delta)$ the IVP (1.1)-(1.2) has a unique solution $x(t, \gamma)$ on $[-r, \alpha]$; 
(ii) there exist a closed subset $M_{1} \subset C$ which is also a bounded and convex subset of $W^{1, \infty}$, $M_{2} \subset \mathbb{R}^{n}$ compact and convex subset and $M_{3} \subset \Theta, M_{4} \subset \Xi$ closed, bounded and convex subsets of the respective spaces such that $x_{t}(\cdot, \gamma) \in M_{1}, x\left(t-\tau\left(t, x_{t}(\cdot, \gamma), \xi\right), \gamma\right) \in M_{2}$, $\theta \in M_{3}$ and $\xi \in M_{4}$ for $\gamma=(\varphi, \theta, \xi) \in P$ and $t \in[0, \alpha]$; and

(iii) $x_{t}(\cdot, \gamma) \in W^{1, \infty}$ for $\gamma \in P$ and $t \in[0, \alpha]$, and there exist constants $N=N(\alpha, \delta)$ and $L=L(\alpha, \delta)$ such that

$$
\left|x_{t}(\cdot, \gamma)\right|_{W^{1, \infty}} \leq N, \quad \gamma \in P, t \in[0, \alpha]
$$

and

$$
\left|x_{t}(\cdot, \gamma)-x_{t}(\cdot, \bar{\gamma})\right|_{W^{1, \infty}} \leq L|\gamma-\bar{\gamma}|_{\Gamma}, \quad \gamma \in P, t \in[0, \alpha]
$$

The following result is obvious.

Remark 3.2 Suppose the conditions of Theorem 3.1 hold, $P$ and $\alpha$ are defined by Theorem 3.1, and let $\mathcal{P}$ denote the subset of $P$ consisting of those parameters which satisfy the compatibility condition, i.e.,

$$
\mathcal{P}:=\left\{(\varphi, \theta, \xi) \in P: \varphi \in C^{1}, \quad \dot{\varphi}(0-)=f(0, \varphi, \varphi(-\tau(0, \varphi, \xi)), \theta)\right\} .
$$

Then for all parameter values $\gamma \in \mathcal{P}$ the corresponding solution $x(t, \gamma)$ is continuously differentiable wrt $t$ for $t \in[-r, \alpha]$.

Throughout the rest of the paper we will use the following notations. The parameter $\hat{\gamma} \in \Pi$ is fixed, and the constants $\delta>0,0<\alpha \leq T$ are defined by Theorem 3.1, and let $P:=\mathcal{B}_{\Gamma}(\hat{\gamma} ; \delta)$. The sets $M_{1} \subset C, M_{2} \subset \mathbb{R}^{n}, M_{3} \subset \Theta$ and $M_{4} \subset \Xi$ are defined by Theorem 3.1 (ii), $L_{1}=$ $L_{1}\left(\alpha, M_{1}, M_{2}, M_{3}\right), L_{2}=L_{2}\left(\alpha, M_{1}, M_{4}\right)$ and $L_{4}=L_{4}\left(\alpha, M_{1}, M_{4}\right)$ denote the corresponding Lipschitz constants from (A1) (ii), (A2) (ii) and (A2) (iv), respectively, and the constants $N=N(\alpha, \delta)$ and $L=L(\alpha, \delta)$ are defined by Theorem 3.1 (iii). We will restrict our attention to the fixed parameter set $P$, so the sets $M_{1}, M_{2}, M_{3}$ and $M_{4}$, and the constants $L_{1}, L_{2}, L_{4}, L$ and $N$ can be considered to be fixed throughout this paper.

Lemma 3.3 Assume (A1) (i), (ii), (A2) (i),(ii), $\gamma=(\varphi, \xi, \theta) \in P, h_{k}=\left(h_{k}^{\varphi}, h_{k}^{\xi}, h_{k}^{\theta}\right) \in \Gamma$ is a sequence such that $\gamma+h_{k} \in P$ for $k \in \mathbb{N}$ and $\left|h_{k}\right|_{\Gamma} \rightarrow 0$ as $k \rightarrow \infty$. Let $x(t):=x(t, \gamma), x^{k}(t):=$ $x\left(t, \gamma+h_{k}\right)$ be the corresponding solutions of the IVP (1.1)-(1.2), and $u^{k}(s):=t-\tau\left(t, x_{t}^{k}, \xi+h_{k}^{\xi}\right)$ and $u(t):=t-\tau\left(t, x_{t}, \xi\right)$. Then there exists $K_{0} \geq 0$ such that

$$
\left|u^{k}(t)-u(t)\right| \leq K_{0}\left|h_{k}\right|_{\Gamma}, \quad t \in[0, \alpha], \quad k \in \mathbb{N} .
$$

If, in addition, (A2) (iv) holds, then $u, u^{k} \in W^{1, \infty}([0, \alpha], \mathbb{R})$, and if (A2) (v) is also satisfied, then there exists $K_{1} \geq 0$ such that

$$
\left|u^{k}-u\right|_{W^{1, \infty}([0, \alpha], \mathbb{R})} \leq K_{1}\left|h_{k}\right|_{\Gamma}, \quad k \in \mathbb{N} .
$$


Proof Assumption (A2) (ii) implies

$$
\left|u^{k}(t)-u(t)\right|=\left|\tau\left(t, x_{t}^{k}, \xi+h_{k}^{\xi}\right)-\tau\left(t, x_{t}, \xi\right)\right| \leq L_{2}\left(\left|x_{t}^{k}-x_{t}\right|_{C}+\left|h_{k}^{\xi}\right|_{\Xi}\right), \quad t \in[0, \alpha],
$$

so (3.2) yields (3.4) with $K_{0}:=L_{2}(L+1)$.

Now assume (A2) (iv) also holds. For simplicity of the notation let $h_{0}:=0=(0,0,0) \in \Gamma$, and so $x^{0}:=x$ and $u^{0}:=u$. Then (A2) (ii), the Mean Value Theorem and (3.1) imply for $k \in \mathbb{N}_{0}$ and $t, \bar{t} \in[0, \alpha]$

$$
\left|\tau\left(t, x_{t}^{k}, \xi+h_{k}^{\xi}\right)-\tau\left(\bar{t}, x_{\bar{t}}^{k}, \xi+h_{k}^{\xi}\right)\right| \leq L_{2}\left(|t-\bar{t}|+\left|x_{t}^{k}-x_{\bar{t}}^{k}\right|_{C}\right) \leq L_{2}(1+N)|t-\bar{t}| .
$$

Hence $u^{k}$ is Lipschitz continuous, and so it is almost everywhere differentiable on $[0, \alpha]$, and $\left|\dot{u}^{k}\right|_{L^{\infty}([0, \alpha], \mathbb{R})} \leq L_{2}(1+N)$. Therefore $u^{k} \in W^{1, \infty}([0, \alpha], \mathbb{R})$ for $k \in \mathbb{N}_{0}$.

Let $L_{4}=L_{4}\left(\alpha, M_{1}, M_{4}\right)$ be defined by (A2) (v). Assumption (A2) (v) and (3.2) give

$$
\left|\dot{u}^{k}(t)-\dot{u}(t)\right|=\left|\frac{d}{d t} \tau\left(t, x_{t}^{k}, \xi+h_{k}^{\xi}\right)-\frac{d}{d t} \tau\left(t, x_{t}, \xi\right)\right| \leq L_{4}\left(\left|x_{t}^{k}-x_{t}\right|_{C}+\left|h_{k}^{\xi}\right| \Xi\right) \leq L_{4}(L+1)\left|h_{k}\right|_{\Gamma}
$$

for a.e. $t \in[0, \alpha]$. Therefore (3.5) holds with $K_{1}:=\max \left\{K_{0}, L_{4}(L+1)\right\}$.

We note that (A2) (v) and (viii) hold under natural assumptions for example for functions of the form

$$
\tau(t, \psi, \xi)=\bar{\tau}\left(t, \psi\left(-\eta^{1}(t)\right), \ldots, \psi\left(-\eta^{\ell}(t)\right), \int_{-r}^{0} A(t, \zeta) \psi(\zeta) d \zeta, \xi(t)\right)
$$

and

$$
f(t, \psi, u, \theta)=\bar{f}\left(t, \psi\left(-\nu^{1}(t)\right), \ldots, \psi\left(-\nu^{m}(t)\right), \int_{-r}^{0} B(t, \zeta) \psi(\zeta) d \zeta, \theta(t)\right) .
$$

Here $\Theta=W^{1, \infty}([0, T], \mathbb{R})$ and $\Xi=W^{1, \infty}([0, T], \mathbb{R})$ can be used, and then we have, e.g., for $\tau$ under straightforward assumptions we have for a.e. $t \in[0, \alpha], y \in W^{1, \infty}\left([-r, \alpha], \mathbb{R}^{n}\right)$

$$
\begin{aligned}
\frac{d}{d t} \tau\left(t, y_{t}, \xi\right)= & D_{1} \bar{\tau}\left(t, y\left(t-\eta^{1}(t)\right), \ldots, y\left(t-\eta^{\ell}(t)\right), \int_{-r}^{0} A(t, \zeta) y(t+\zeta) d \zeta, \xi(t)\right) \\
& +\sum_{i=1}^{\ell} D_{i+1} \bar{\tau}\left(t, y\left(t-\eta^{1}(t)\right), \ldots, y\left(t-\eta^{\ell}(t)\right), \int_{-r}^{0} A(t, \zeta) y(t+\zeta) d \zeta, \xi(t)\right) \\
& \quad \times \dot{y}\left(t-\eta^{i}(t)\right)\left(1-\dot{\eta}^{i}(t)\right) \\
& +D_{i+2} \bar{\tau}\left(t, y\left(t-\eta^{1}(t)\right), \ldots, y\left(t-\eta^{\ell}(t)\right), \int_{-r}^{0} A(t, \zeta) y(t+\zeta) d \zeta, \xi(t)\right) \\
& \quad \times \int_{-r}^{0}\left[D_{1} A(t, \zeta) y(t+\zeta)+A(t, \zeta) \dot{y}(t+\zeta)\right] d \zeta \\
& +D_{i+3} \bar{\tau}\left(t, y\left(t-\eta^{1}(t)\right), \ldots, y\left(t-\eta^{\ell}(t)\right), \int_{-r}^{0} A(t, \zeta) y(t+\zeta) d \zeta, \xi(t)\right) \dot{\xi}(t) .
\end{aligned}
$$

Similar formula holds for $\frac{d}{d t} f\left(t, y_{t}, y\left(t-\tau\left(t, y_{t}, \xi\right)\right), \theta\right)$. So if $\bar{\tau}$ and $\bar{f}$ are continuously differentiable, $\eta^{i}$ are continuously differentiable and $\operatorname{ess}_{\sup } \operatorname{su}_{t \in, T]}\left(1-\dot{\eta}^{i}(t)\right)>0$ for $i=1, \ldots, \ell$, then it is easy to argue that (A2) (v) and (viii) hold. 


\section{First-order differentiability wrt the parameters}

In this section we study the differentiability of the solution $x(t, \gamma)$ of the IVP (1.1)-(1.2) wrt $\gamma$. The proof of our differentiability results will be based on the following lemmas.

Lemma 4.1 Let $y \in W^{1, \infty}\left([-r, \alpha], \mathbb{R}^{n}\right), \omega_{k} \in(0, \infty)(k \in \mathbb{N})$ be a sequence satisfying $\omega_{k} \rightarrow 0$ as $k \rightarrow \infty$. Let $u, u^{k} \in \mathcal{P} \mathcal{M}([0, \alpha],[-r, \alpha])(k \in \mathbb{N})$ be such that

$$
\left|u^{k}-u\right|_{W^{1, \infty}([0, \alpha], \mathbb{R})} \leq \omega_{k}, \quad k \in \mathbb{N} .
$$

Then

$$
\lim _{k \rightarrow \infty} \frac{1}{\omega_{k}} \int_{0}^{\alpha}\left|y\left(u^{k}(s)\right)-y(u(s))-\dot{y}(u(s))\left(u^{k}(s)-u(s)\right)\right| d s=0 .
$$

Proof Let $0=t_{0}<t_{1}<\cdots<t_{m-1}<t_{m}=\alpha$ be the mesh points of $u$ from the Definition 2.6, and let $0<\varepsilon<\min \left\{t_{i+1}-t_{i}: i=0, \ldots, m-1\right\} / 2$ be fixed, and introduce $t_{i}^{\prime}:=t_{i}+\varepsilon$ for $i=0, \ldots, m-1, t_{i}^{\prime \prime}:=t_{i}-\varepsilon$ for $i=1, \ldots, m, t_{0}^{\prime \prime}:=0, t_{m}^{\prime}:=\alpha$, and let

$$
M:=\min _{i=0, \ldots, m-1} \operatorname{essinf}_{t \in\left[t_{i}^{\prime}, t_{i+1}^{\prime \prime}\right]}|\dot{u}(t)| \text {. }
$$

We have $M>0$, since $u \in \mathcal{P} \mathcal{M}([0, \alpha],[-r, \alpha])$. Assumption (4.1) yields that there exists $k_{0}>0$ such that $\left|u^{k}-u\right|_{W^{1, \infty}([0, \alpha], \mathbb{R})}<\frac{M}{2}$ for $k \geq k_{0}$. Then for $k \geq k_{0}$ it follows $\left|\dot{u}^{k}(s)\right| \geq \frac{M}{2}$ and $\left|\dot{u}(s)+\nu\left(\dot{u}^{k}(s)-\dot{u}(s)\right)\right| \geq \frac{M}{2}$ for a.e. $s \in\left[t_{i}^{\prime}, t_{i+1}^{\prime \prime}\right], i=0, \ldots, m-1$ and $\nu \in[0,1]$. Let $A:=|y|_{W^{1, \infty}}\left([-r, \alpha], \mathbb{R}^{n}\right)$. Then simple manipulations, (4.1) and Fubini's theorem yield

$$
\begin{aligned}
\int_{0}^{\alpha} \mid y\left(u^{k}(s)\right)- & y(u(s))-\dot{y}(u(s))\left(u^{k}(s)-u(s)\right) \mid d s \\
\leq & \sum_{i=0}^{m} \int_{t_{i}^{\prime \prime}}^{t_{i}^{\prime}}\left(\left|y\left(u^{k}(s)\right)-y(u(s))\right|+|\dot{y}(u(s))|\left|u^{k}(s)-u(s)\right|\right) d s \\
& \quad+\sum_{i=0}^{m-1} \int_{t_{i}^{\prime}}^{t_{i+1}^{\prime \prime}}\left|\int_{u(s)}^{u^{k}(s)}(\dot{y}(v)-\dot{y}(u(s))) d v\right| d s \\
\leq & (m+1) 2 \varepsilon 2 A\left|u^{k}-u\right| C([0, \alpha], \mathbb{R}) \\
& \quad+\sum_{i=0}^{m-1} \int_{t_{i}^{\prime}}^{t_{i+1}^{\prime \prime}}\left|\int_{0}^{1}\left[\dot{y}\left(u(s)+\nu\left(u^{k}(s)-u(s)\right)\right)-\dot{y}(u(s))\right]\left(u^{k}(s)-u(s)\right) d \nu\right| d s \\
\leq \quad & \omega_{k}\left[(m+1) 4 A \varepsilon+\sum_{i=0}^{m-1} \int_{0}^{1} \int_{t_{i}^{\prime}}^{t_{i+1}^{\prime \prime}}\left|\dot{y}\left(u(s)+\nu\left(u^{k}(s)-u(s)\right)\right)-\dot{y}(u(s))\right| d s d \nu\right] .
\end{aligned}
$$

It follows from Lemma 2.3 and Remark 2.4 that for every $\nu \in[0,1]$

$$
\lim _{k \rightarrow \infty} \int_{t_{i}^{\prime}}^{t_{i+1}^{\prime \prime}}\left|\dot{y}\left(u(s)+\nu\left(u^{k}(s)-u(s)\right)\right)-\dot{y}(u(s))\right| d s=0, \quad i=0, \ldots, m-1,
$$

hence we get by using the Lebesgue's Dominated Convergence Theorem that

$$
\limsup _{k \rightarrow \infty} \frac{1}{\omega_{k}} \int_{0}^{\alpha}\left|y\left(u^{k}(s)\right)-y(u(s))-\dot{y}(u(s))\left(u^{k}(s)-u(s)\right)\right| d s \leq(m+1) 4 A \varepsilon .
$$

This concludes the proof of (4.2), since $\varepsilon>0$ can be arbitrary close to 0 . 
We introduce the notations

$$
\begin{aligned}
\omega_{f}(t, \bar{\psi}, \bar{u}, \bar{\theta}, \psi, u, \theta):= & f(t, \psi, u, \theta)-f(t, \bar{\psi}, \bar{u}, \bar{\theta})-D_{2} f(t, \bar{\psi}, \bar{u}, \bar{\theta})(\psi-\bar{\psi}) \\
& -D_{3} f(t, \bar{\psi}, \bar{u}, \bar{\theta})(u-\bar{u})-D_{4} f(t, \bar{\psi}, \bar{u}, \bar{\theta})(\theta-\bar{\theta}), \\
\omega_{\tau}(t, \bar{\psi}, \bar{\xi}, \psi, \xi):= & \tau(t, \psi, \xi)-\tau(t, \bar{\psi}, \bar{\xi})-D_{2} \tau(t, \bar{\psi}, \bar{\xi})(\psi-\bar{\psi}) \\
& -D_{3} \tau(t, \bar{\psi}, \bar{\xi})(\xi-\bar{\xi})
\end{aligned}
$$

for $t \in[0, T], \bar{\psi}, \psi \in \Omega_{1}, \bar{u}, u \in \Omega_{2}, \bar{\theta}, \theta \in \Omega_{3}, \bar{\xi}, \xi \in \Omega_{4}$, and

$$
\begin{gathered}
\Omega_{f}(\varepsilon):=\max _{i=2,3,4} \sup \left\{\left|D_{i} f(t, \psi, u, \theta)-D_{i} f(t, \tilde{\psi}, \tilde{u}, \tilde{\theta})\right|_{\mathcal{L}\left(Y_{i}, \mathbb{R}^{n}\right)}\right. \\
|\psi-\tilde{\psi}|_{C}+|u-\tilde{u}|+|\theta-\tilde{\theta}|_{\Theta} \leq \varepsilon, \quad t \in[0, \alpha], \psi, \tilde{\psi} \in M_{1}, \\
\left.u, \tilde{u} \in M_{2}, \theta, \tilde{\theta} \in M_{3}\right\} \\
\Omega_{\tau}(\varepsilon):=\max _{i=2,3} \sup \left\{\left|D_{i} \tau(t, \psi, \xi)-D_{i} \tau(t, \bar{\psi}, \bar{\xi})\right|_{\mathcal{L}\left(Z_{i}, \mathbb{R}\right)}:|\psi-\bar{\psi}|_{C}+|\xi-\bar{\xi}|_{\Xi} \leq \varepsilon,\right. \\
\left.t \in[0, \alpha], \psi, \bar{\psi} \in M_{1}, \xi, \bar{\xi} \in M_{4}\right\},
\end{gathered}
$$

where $Y_{2}:=C, Y_{3}:=\mathbb{R}^{n}, Y_{4}:=\Theta, Z_{2}:=C$ and $Z_{3}:=\Xi$.

The following result is an easy generalization of Lemma 4.2 of [12] for the IVP (1.1)-(1.2), therefore we omit its proof here. (See also the related proof of Lemma 5.8 below.)

Lemma 4.2 (see [12]) Suppose (A1) (i)-(iii), (A2) (i)-(iii). Let $P$ and $\alpha>0$ be defined by Theorem 3.1, let $\gamma=(\varphi, \theta, \xi) \in P$ be fixed, and $h_{k}=\left(h_{k}^{\varphi}, h_{k}^{\theta}, h_{k}^{\xi}\right) \in \Gamma(k \in \mathbb{N})$ be a sequence satisfying $\left|h_{k}\right|_{\Gamma} \rightarrow 0$ as $k \rightarrow \infty$, and $\gamma+h_{k} \in P$ for $k \in \mathbb{N}$. Let $x(t):=x(t, \gamma)$, $x^{k}(t):=x\left(t, \gamma+h_{k}\right), u(t):=t-\tau\left(t, x_{t}, \xi\right)$ and $u^{k}(t):=t-\tau\left(t, x^{k}, \xi+h_{k}^{\xi}\right)$. Then

$$
\lim _{k \rightarrow \infty} \frac{1}{\left|h_{k}\right|_{\Gamma}} \int_{0}^{\alpha}\left|\omega_{f}\left(s, x_{s}, x(u(s)), \theta, x_{s}^{k}, x^{k}\left(u^{k}(s)\right), \theta+h_{k}^{\theta}\right)\right| d s=0
$$

and

$$
\lim _{k \rightarrow \infty} \frac{1}{\left|h_{k}\right|_{\Gamma}} \int_{0}^{\alpha}\left|\omega_{\tau}\left(s, x_{s}, \xi, x_{s}^{k}, \xi+h_{k}^{\xi}\right)\right| d s=0
$$

A solution $x(\cdot, \gamma)$ of the IVP (1.1)-(1.2) for $\gamma \in P$ is, in general, only a $W^{1, \infty}$-function on the interval $[-r, 0]$, but it is continuously differentiable for $t \geq 0$. In [16] (see also [12]) a parameter set

$$
P_{1}:=\{\gamma=(\varphi, \theta, \xi) \in P: x(\cdot, \gamma) \in X(\alpha, \xi)\}
$$

was considered, where

$$
\begin{gathered}
X(\alpha, \xi):=\left\{x \in W^{1, \infty}\left([-r, \alpha], \mathbb{R}^{n}\right): x_{t} \in \Omega_{1}, x\left(t-\tau\left(t, x_{t}, \xi\right)\right) \in \Omega_{2} \text { for } t \in[0, \alpha],\right. \\
\text { and } \left.\operatorname{essinf}\left\{\frac{d}{d t}\left(t-\tau\left(t, x_{t}, \xi\right)\right): \text { a.e. } t \in\left[0, \alpha^{*}\right]\right\}>0\right\}
\end{gathered}
$$

and $\alpha^{*}:=\min \{r, \alpha\}$. Then Lemma 2.3 yields that the function $t \mapsto \dot{x}\left(t-\tau\left(t, x_{t}, \xi\right)\right)$ is welldefined for a.e. $t \in\left[0, \alpha^{*}\right]$ and it is integrable on $\left[0, \alpha^{*}\right]$, and it is well-defined and continuous on 
$\left[\alpha^{*}, \alpha\right]$. Note that it was shown in [16] (see also [12]) that $P_{1}$ is an open subset of the parameter set $P$. In this section we relax this condition. We define the parameter set

$$
\begin{gathered}
P_{2}:=\left\{\gamma=(\varphi, \theta, \xi) \in P: \text { the map }\left[0, \alpha^{*}\right] \rightarrow \mathbb{R}, t \mapsto t-\tau\left(t, x_{t}(\cdot, \gamma), \xi\right)\right. \\
\text { belongs to } \left.\mathcal{P} \mathcal{M}\left(\left[0, \alpha^{*}\right],\left[-r, \alpha^{*}\right]\right)\right\} .
\end{gathered}
$$

Then we have $P_{1} \subset P_{2} \subset P$, and Lemma 2.7 yields that for a solution $x$ corresponding to parameter $\gamma \in P_{2}$ the function $t \mapsto \dot{x}\left(t-\tau\left(t, x_{t}, \xi\right)\right)$ is well-defined for a.e. $t \in\left[0, \alpha^{*}\right]$ and it is integrable on $\left[0, \alpha^{*}\right]$. Therefore, as the next discussion will show, the parameter set where the variational equation is defined, and correspondingly the differentiability of the solution wrt the parameters can be obtained is larger than in the previous papers $[9,12,16]$.

Let $\gamma=(\varphi, \theta, \xi) \in P_{2}$ be fixed, and let $x(t):=x(t, \gamma)$. Consider the space $C \times \Theta \times \Xi$ equipped with the product norm $\left|\left(h^{\varphi}, h^{\theta}, h^{\xi}\right)\right|_{C \times \Theta \times \Xi}:=\left|h^{\varphi}\right|_{C}+\left|h^{\theta}\right|_{\Theta}+\left|h^{\xi}\right| \Xi$. Then for a.e. $t \in[0, \alpha]$ we introduce the linear operator $L(t, x): C \times \Theta \times \Xi \rightarrow \mathbb{R}^{n}$ by

$$
\begin{aligned}
L(t, x)\left(h^{\varphi},\right. & \left.h^{\theta}, h^{\xi}\right) \\
:= & D_{2} f\left(t, x_{t}, x\left(t-\tau\left(t, x_{t}, \xi\right)\right), \theta\right) h^{\varphi}+D_{3} f\left(t, x_{t}, x\left(t-\tau\left(t, x_{t}, \xi\right)\right), \theta\right) \\
& \quad \times\left[-\dot{x}\left(t-\tau\left(t, x_{t}, \xi\right)\right)\left(D_{2} \tau\left(t, x_{t}, \xi\right) h^{\varphi}+D_{3} \tau\left(t, x_{t}, \xi\right) h^{\xi}\right)+h^{\varphi}\left(-\tau\left(t, x_{t}, \xi\right)\right)\right] \\
& +D_{4} f\left(t, x_{t}, x\left(t-\tau\left(t, x_{t}, \xi\right)\right), \theta\right) h^{\theta}
\end{aligned}
$$

for $\left(h^{\varphi}, h^{\theta}, h^{\xi}\right) \in C \times \Theta \times \Xi$. We have by (A1) (ii), (A2) (ii) and (3.1)

$$
\begin{aligned}
\left|L(t, x)\left(h^{\varphi}, h^{\theta}, h^{\xi}\right)\right| & \leq L_{1}\left|h^{\varphi}\right|_{C}+L_{1}\left[N\left(L_{2}\left|h^{\varphi}\right|_{C}+L_{2}\left|h^{\xi}\right|_{\Xi}\right)+\left|h^{\varphi}\right|_{C}\right]+L_{1}\left|h^{\theta}\right|_{\Theta} \\
& \leq L_{1} N_{0}\left|\left(h^{\varphi}, h^{\theta}, h^{\xi}\right)\right|_{C \times \Theta \times \Xi}, \quad \text { a.e. } t \in[0, \alpha],
\end{aligned}
$$

where

$$
N_{0}:=N L_{2}+3
$$

Therefore

$$
|L(t, x)|_{\mathcal{L}\left(C \times \Theta \times \Xi, \mathbb{R}^{n}\right)} \leq L_{1} N_{0}, \quad \text { a.e. } t \in[0, \alpha] .
$$

Hence $L(t, x)$ is a bounded linear operator for all $t$ for which $\dot{x}\left(t-\tau\left(t, x_{t}, \xi\right)\right)$ exists, i.e., for a.e. $t \in[0, \alpha]$.

For $\gamma \in P_{2}$ we define the variational equation associated to $x=x(\cdot, \gamma)$ as

$$
\begin{aligned}
\dot{z}(t) & =L(t, x)\left(z_{t}, h^{\theta}, h^{\xi}\right) \quad \text { a.e. } t \in[0, \alpha], \\
z(t) & =h^{\varphi}(t), \quad t \in[-r, 0],
\end{aligned}
$$

where $h=\left(h^{\varphi}, h^{\theta}, h^{\xi}\right) \in C \times \Theta \times \Xi$ is fixed. The IVP (4.13)-(4.14) is a Carathéodory type linear delay equation. By its solution we mean a continuous function $z:[-r, \alpha] \rightarrow \mathbb{R}^{n}$, which is absolutely continuous on $[0, \alpha]$, and it satisfies (4.13) for a.e. $t \in[0, \alpha]$ and (4.14) for all $t \in[-r, 0]$. Standard argument ([3], [7]) shows that the IVP (4.13)-(4.14) has a unique solution $z(t)=z(t, \gamma, h)$ for $t \in[-r, \alpha], \gamma \in P_{2}$ and $h=\left(h^{\varphi}, h^{\theta}, h^{\xi}\right) \in C \times \Theta \times \Xi$.

The following result was proved in [12] for the parameter set $P_{1}$ (see Lemma 4.4 in [12]), but the proof is identical for the parameter set $P_{2}$, as well.

Lemma 4.3 (see [12]) Assume (A1) (i)-(iii), (A2) (i)-(iii). Let $\gamma \in P_{2}$, and $x(t):=x(t, \gamma)$ for $t \in[-r, \alpha]$. Let $h \in C \times \Theta \times \Xi$ and let $z(t, \gamma, h)$ be the corresponding solution of the IVP (4.13)-(4.14) on $[-r, \alpha]$. Then 
(i) $z(t, \gamma, \cdot) \in \mathcal{L}\left(C \times \Theta \times \Xi, \mathbb{R}^{n}\right)$, the map $C \times \Theta \times \Xi \rightarrow C, h \mapsto z_{t}(\cdot, \gamma, h)$ is in $\mathcal{L}(C \times \Theta \times \Xi, C)$, and

$$
|z(t, \gamma, h)| \leq\left|z_{t}(\cdot, \gamma, h)\right|_{C} \leq N_{1}|h|_{C \times \Theta \times \Xi, \quad t \in[0, \alpha], \gamma \in P_{2}, h \in C \times \Theta \times \Xi}
$$

where $N_{1}:=e^{L_{1} N_{0} \alpha}$;

(ii) there exists $N_{2} \geq 0$ such that

$$
\left|z_{t}(\cdot, \gamma, h)\right|_{W^{1, \infty}} \leq N_{2}|h|_{\Gamma}, \quad t \in[0, \alpha], \gamma \in P_{2}, h \in \Gamma .
$$

Next we show that the linear operators $z(t, \gamma, \cdot)$ and $z_{t}(\cdot, \gamma, \cdot)$ are continuous in $t$ and $\gamma$, assuming that $\gamma$ belongs to $P_{2}$. First we need the following result.

Lemma 4.4 Assume (A1) (i)-(iii), (A2) (i)-(iii). Let $\gamma \in P_{2}, h=\left(h^{\varphi}, h^{\theta}, h^{\xi}\right) \in \Gamma, h_{k}=$ $\left(h_{k}^{\varphi}, h_{k}^{\theta}, h_{k}^{\xi}\right) \in \Gamma(k \in \mathbb{N})$ be a sequence such that $\left|h_{k}\right|_{\Gamma} \rightarrow 0$ as $k \rightarrow \infty$, and $\gamma+h_{k} \in P_{2}$ for $k \in \mathbb{N}$. Let $x(s):=x(s, \gamma), x^{k}(s):=x\left(s, \gamma+h_{k}\right), u(s):=s-\tau\left(s, x_{s}, \xi\right)$, and $u^{k}(s):=s-\tau\left(s, x_{s}^{k}, \xi+h_{k}^{\xi}\right)$. Then there exists a nonnegative sequence $c_{0, k}$ such that $c_{0, k} \rightarrow 0$ as $k \rightarrow \infty$, and

$$
\left|L\left(s, x^{k}\right) h-L(s, x) h\right| \leq c_{0, k}|h|_{\Gamma}+L_{1} L_{2}\left|\dot{x}\left(u^{k}(s)\right)-\dot{x}(u(s))\right||h|_{\Gamma}
$$

for a.e. $s \in[0, \alpha], k \in \mathbb{N}$ and $h \in \Gamma$.

Proof We have

$$
\begin{aligned}
L\left(s, x^{k}\right)\left(h^{\varphi}, h^{\theta}, h^{\xi}\right)-L(s, x)\left(h^{\varphi}, h^{\theta}, h^{\xi}\right) & \left(D_{2} f\left(s, x_{s}^{k}, x^{k}\left(u^{k}(s)\right), \theta+h_{k}^{\theta}\right)-D_{2} f\left(s, x_{s}, x(u(s)), \theta\right)\right) h^{\varphi} \\
+ & \left(D_{3} f\left(s, x_{s}^{k}, x^{k}\left(u^{k}(s)\right), \theta+h_{k}^{\theta}\right)-D_{3} f\left(s, x_{s}, x(u(s)), \theta\right)\right) \\
& \times\left(-\dot{x}^{k}\left(u^{k}(s)\right)\right)\left(D_{2} \tau\left(s, x_{s}^{k}, \xi+h_{k}^{\xi}\right) h^{\varphi}+D_{3} \tau\left(s, x_{s}^{k}, \xi+h_{k}^{\xi}\right) h^{\xi}\right) \\
+ & \left.D_{3} f\left(s, x_{s}, x(u(s)), \theta\right)\left(-\dot{x}^{k}\left(u^{k}(s)\right)+\dot{x}\left(u^{k}(s)\right)\right)\right) \\
& \times\left(D_{2} \tau\left(s, x_{s}^{k}, \xi+h_{k}^{\xi}\right) h^{\varphi}+D_{3} \tau\left(s, x_{s}^{k}, \xi+h_{k}^{\xi}\right) h^{\xi}\right) \\
+ & \left.D_{3} f\left(s, x_{s}, x(u(s)), \theta\right)\left(-\dot{x}\left(u^{k}(s)\right)+\dot{x}(u(s))\right)\right) \\
& \times\left(D_{2} \tau\left(s, x_{s}^{k}, \xi+h_{k}^{\xi}\right) h^{\varphi}+D_{3} \tau\left(s, x_{s}^{k}, \xi+h_{k}^{\xi}\right) h^{\xi}\right) \\
+ & D_{3} f\left(s, x_{s}, x(u(s)), \theta\right)(-\dot{x}(u(s))) \\
& \times\left[\left(D_{2} \tau\left(s, x_{s}^{k}, \xi+h_{k}^{\xi}\right)-D_{2} \tau\left(s, x_{s}, \xi\right)\right) h^{\varphi}\right. \\
& \left.\quad+\left(D_{3} \tau\left(s, x_{s}^{k}, \xi+h_{k}^{\xi}\right)-D_{3} \tau\left(s, x_{s}, \xi\right)\right) h^{\xi}\right] \\
+ & \left(D_{3} f\left(s, x_{s}^{k}, x^{k}\left(u^{k}(s)\right), \theta+h_{k}^{\theta}\right)-D_{3} f\left(s, x_{s}, x(u(s)), \theta\right)\right) h^{\varphi}\left(-\tau\left(s, x_{s}^{k}, \xi+h_{k}^{\xi}\right)\right) \\
+ & D_{3} f\left(s, x_{s}, x(u(s)), \theta\right)\left(h^{\varphi}\left(-\tau\left(s, x_{s}^{k}, \xi+h_{k}^{\xi}\right)\right)-h^{\varphi}\left(-\tau\left(s, x_{s}, \xi\right)\right)\right) \\
+ & \left(D_{4} f\left(s, x_{s}^{k}, x^{k}\left(u^{k}(s)\right), \theta+h_{k}^{\theta}\right)-D_{4} f\left(s, x_{s}, x(u(s)), \theta\right)\right) h^{\theta}, \\
&
\end{aligned}
$$


Relations (3.1), (3.2), (3.4) and the Mean Value Theorem give

$$
\begin{aligned}
\left|x^{k}\left(u^{k}(s)\right)-x(u(s))\right| & \leq\left|x^{k}\left(u^{k}(s)\right)-x\left(u^{k}(s)\right)\right|+\left|x\left(u^{k}(s)\right)-x(u(s))\right| \\
& \leq L\left|h_{k}\right|_{\Gamma}+N\left|u^{k}(s)-u(s)\right| \\
& \leq K_{2}\left|h_{k}\right|_{\Gamma},
\end{aligned}
$$

with $K_{2}:=L+N K_{0}$

$$
\left|x_{s}^{k}-x_{s}\right|_{C}+\left|x^{k}\left(u^{k}(s)\right)-x(u(s))\right|+\left|h_{k}^{\theta}\right|_{\Theta} \leq K_{3}\left|h_{k}\right|_{\Gamma},
$$

with $K_{3}:=L+K_{2}+1$, and

$$
\left|x_{s}^{k}-x_{s}\right|_{C}+\left|h_{k}^{\xi}\right|_{\Xi} \leq(L+1)\left|h_{k}\right|_{\Gamma} .
$$

Combining the above estimates with (A1) (ii), (A2) (ii), (3.1), (3.2), (3.4) and the definition of $\Omega_{f}$ and $\Omega_{\tau}$ we get

$$
\begin{aligned}
\mid L\left(s, x^{k}\right)\left(h^{\varphi}\right. & \left., h^{\theta}, h^{\xi}\right)-L(s, x)\left(h^{\varphi}, h^{\theta}, h^{\xi}\right) \mid \\
\leq & \Omega_{f}\left(K_{3}\left|h_{k}\right|_{\Gamma}\right)\left|h^{\varphi}\right|_{C}+\Omega_{f}\left(K_{3}\left|h_{k}\right|_{\Gamma}\right) N L_{2}\left(\left|h^{\varphi}\right|_{C}+\left|h^{\xi}\right|_{\Xi}\right) \\
& +L_{1} L\left|h_{k}\right|_{\Gamma} L_{2}\left(\left|h^{\varphi}\right|_{C}+\left|h^{\xi}\right|_{\Xi}\right)+L_{1}\left|\dot{x}\left(u^{k}(s)\right)-\dot{x}(u(s))\right| L_{2}\left(\left|h^{\varphi}\right|_{C}+\left|h^{\xi}\right|_{\Xi}\right) \\
& \left.+L_{1} N \Omega_{\tau}\left(\left.(L+1) h_{k}\right|_{\Gamma}\right)\right)\left(\left|h^{\varphi}\right|_{C}+\left|h^{\xi}\right|_{\Xi}\right)+\Omega_{f}\left(K_{3}\left|h_{k}\right|_{\Gamma}\right)\left|h^{\varphi}\right|_{C} \\
& +L_{1}\left|\dot{h}^{\varphi}\right|_{L^{\infty}} K_{0}\left|h_{k}\right|_{\Gamma}+\Omega_{f}\left(K_{3}\left|h_{k}\right|_{\Gamma}\right)\left|h^{\theta}\right|_{\Theta}, \quad s \in[0, \alpha]
\end{aligned}
$$

which yields (4.17) with $c_{0, k}:=N_{0} \Omega_{f}\left(K_{3}\left|h_{k}\right|_{\Gamma}\right)+L_{1} L_{2} L\left|h_{k}\right|_{\Gamma}+L_{1} N \Omega_{\tau}\left((L+1)\left|h_{k}\right|_{\Gamma}\right)+$ $L_{1} K_{0}\left|h_{k}\right|_{\Gamma}$, where $N_{0}$ is defined by (4.12).

Lemma 4.5 Assume (A1) (i)-(iii), (A2) (i)-(v). Let $\gamma \in P_{2}$, and $x(t):=x(t, \gamma)$ for $t \in[-r, \alpha]$. Let $h \in C \times \Omega \times \Xi$ and let $z(t, \gamma, h)$ be the corresponding solution of the IVP (4.13)-(4.14) on $[-r, \alpha]$. Then the maps

$$
\mathbb{R} \times \Gamma \supset[0, \alpha] \times P_{2} \rightarrow \mathcal{L}\left(\Gamma, \mathbb{R}^{n}\right), \quad(t, \gamma) \mapsto z(t, \gamma, \cdot)
$$

and

$$
\mathbb{R} \times \Gamma \supset[0, \alpha] \times P_{2} \rightarrow \mathcal{L}(\Gamma, C), \quad(t, \gamma) \mapsto z_{t}(\cdot, \gamma, \cdot)
$$

are continuous.

Proof Let $\gamma \in P_{2}$ be fixed, and let $h_{k}=\left(h_{k}^{\varphi}, h_{k}^{\theta}, h_{k}^{\xi}\right) \in \Gamma(k \in \mathbb{N})$ be a sequence such that $\left|h_{k}\right|_{\Gamma} \rightarrow 0$ as $k \rightarrow \infty$ and $\gamma+h_{k} \in P_{2}$ for $k \in \mathbb{N}$. For a fixed $h=\left(h^{\varphi}, h^{\theta}, h^{\xi}\right) \in \Gamma$ we define the short notations $x^{k}(t):=x\left(t, \gamma+h_{k}\right), x(t):=x(t, \gamma), u^{k}(t):=t-\tau\left(t, x_{t}^{k}, \xi+h_{k}^{\xi}\right)$, $u(t):=t-\tau\left(t, x_{t}, \xi\right), z^{k, h}(t):=z\left(t, \gamma+h_{k}, h\right)$ and $z^{h}(t):=z(t, \gamma, h)$. The functions $z^{k, h}$ and $z^{h}$ satisfy

$$
\begin{aligned}
z^{k, h}(t) & =h^{\varphi}(0)+\int_{0}^{t} L\left(s, x^{k}\right)\left(z_{s}^{k, h}, h^{\theta}, h^{\xi}\right) d s, \quad t \in[0, \alpha] \\
z^{h}(t) & =h^{\varphi}(0)+\int_{0}^{t} L(s, x)\left(z_{s}^{h}, h^{\theta}, h^{\xi}\right) d s, \quad t \in[0, \alpha]
\end{aligned}
$$


and therefore for $t \in[0, \alpha]$

$$
\left|z^{k, h}(t)-z^{h}(t)\right| \leq \int_{0}^{t}\left|\left(L\left(s, x^{k}\right)-L(s, x)\right)\left(z_{s}^{h}, h^{\theta}, h^{\xi}\right)+L\left(s, x^{k}\right)\left(z_{s}^{k, h}-z_{s}^{h}, 0,0\right)\right| d s .
$$

We have by (4.16) and $N_{2} \geq 1$

$$
\left|\left(z_{s}^{h}, h^{\theta}, h^{\xi}\right)\right|_{\Gamma} \leq N_{2}|h|_{\Gamma}+\left|h^{\theta}\right|_{\Theta}+\left|h^{\xi}\right|_{\Xi} \leq\left(N_{2}+1\right)|h|_{\Gamma} .
$$

Then (4.11), (4.17), (4.21) and (4.22) imply

$$
\left|z^{k, h}(t)-z^{h}(t)\right| \leq c_{1, k}|h|_{\Gamma}+\int_{0}^{t} L_{1} N_{0}\left|z_{s}^{k, h}-z_{s}^{h}\right|_{C} d s, \quad t \in[0, \alpha],
$$

where $c_{1, k}$ is defined by

$$
c_{1, k}:=\alpha c_{0, k}\left(N_{2}+1\right)+L_{1} L_{2}\left(N_{2}+1\right) \int_{0}^{\alpha}\left|\dot{x}\left(u^{k}(s)\right)-\dot{x}(u(s))\right| d s .
$$

Relation 3.5 and Lemma 2.8 yield that

$$
\lim _{k \rightarrow \infty} \int_{\alpha}^{\alpha}\left|\dot{x}\left(u^{k}(s)\right)-\dot{x}(u(s))\right| d s=0 .
$$

Hence $c_{1, k} \rightarrow 0$ as $k \rightarrow \infty$.

Lemma 2.1 is applicable for (4.23) since $\left|z_{0}^{k, h}-z_{0}^{h}\right|_{C}=0$, and it gives

$$
\left|z^{k, h}(t)-z^{h}(t)\right| \leq\left|z_{t}^{k, h}-z_{t}^{h}\right|_{C} \leq c_{1, k} N_{1}|h|_{\Gamma}, \quad t \in[0, \alpha],
$$

where $N_{1}:=e^{L_{1} N_{0} \alpha}$. Therefore we get for $t \in[0, \alpha]$

$$
\left|z\left(t, \gamma+h_{k}, \cdot\right)-z(t, \gamma, \cdot)\right|_{\mathcal{L}\left(W^{1, \infty}, \mathbb{R}^{n}\right)} \leq\left|z_{t}\left(\cdot, \gamma+h_{k}, \cdot\right)-z_{t}(\cdot, \gamma, \cdot)\right|_{\mathcal{L}\left(W^{1, \infty}, C\right)} \leq c_{1, k} N_{1}
$$

for all $k \in \mathbb{N}$.

Let $t \in[0, \alpha]$ be fixed, and let $\nu_{k}$ be a sequence of real numbers such that $t+\nu_{k} \in[0, \alpha]$ for $k \in \mathbb{N}$ and $\nu_{k} \rightarrow 0$ as $k \rightarrow \infty$. Then (4.16) and the Mean Value Theorem yield

$$
\left|z_{t+\nu_{k}}\left(\cdot, \gamma+h_{k}, \cdot\right)-z_{t}\left(\cdot, \gamma+h_{k}, \cdot\right)\right|_{\mathcal{L}(\Gamma, C)} \leq N_{2}\left|\nu_{k}\right|, \quad k \geq k_{0}
$$

Combining this relation with (4.26) and $c_{1, k} \rightarrow 0$ we get

$$
\begin{aligned}
\mid z(t+ & \left.\nu_{k}, \gamma+h_{k}, \cdot\right)-\left.z(t, \gamma, \cdot)\right|_{\mathcal{L}\left(\Gamma, \mathbb{R}^{n}\right)} \\
& \leq\left|z_{t+\nu_{k}}\left(\cdot, \gamma+h_{k}, \cdot\right)-z_{t}(\cdot, \gamma, \cdot)\right|_{\mathcal{L}(\Gamma, C)} \\
& \leq\left|z_{t+\nu_{k}}\left(\cdot, \gamma+h_{k}, \cdot\right)-z_{t}\left(\cdot, \gamma+h_{k}, \cdot\right)\right|_{\mathcal{L}(\Gamma, C)}+\left|z_{t}\left(\cdot, \gamma+h_{k}, \cdot\right)-z_{t}(\cdot, \gamma, \cdot)\right|_{\mathcal{L}(\Gamma, C)} \\
& \leq N_{2}\left|\nu_{k}\right|+c_{1, k} N_{1} \\
& \rightarrow 0, \quad \text { as } k \rightarrow \infty .
\end{aligned}
$$

This completes the proof. 
Remark 4.6 Note that if in the statement of Lemma 4.5 the parameter set $P_{2}$ is replaced by the smaller set $P_{1}$, then assumptions (A2) (iv) and (v) are not needed to prove the statement, since in this case (3.4) and Lemma 2.3 can be used to show that $c_{1, k} \rightarrow 0$ as $k \rightarrow \infty$.

Now we are ready to prove the Fréchet-differentiability of the function $x(t, \gamma)$ wrt $\gamma$. We will denote this derivative by $D_{2} x(t, \gamma)$.

Theorem 4.7 Assume (A1) (i)-(iii), (A2) (i)-(v), and let $P_{2}$ be defined by (4.9). Then the functions

$$
\mathbb{R} \times \Gamma \supset[0, \alpha] \times P \rightarrow \mathbb{R}^{n}, \quad(t, \gamma) \mapsto x(t, \gamma)
$$

and

$$
\mathbb{R} \times \Gamma \supset[0, \alpha] \times P \rightarrow C, \quad(t, \gamma) \mapsto x_{t}(\cdot, \gamma)
$$

are both differentiable wrt $\gamma$ for every $\gamma \in P_{2}$, and

$$
D_{2} x(t, \gamma) h=z(t, \gamma, h), \quad h \in \Gamma, t \in[0, \alpha], \gamma \in P_{2},
$$

and

$$
D_{2} x_{t}(\cdot, \gamma) h=z_{t}(\cdot, \gamma, h), \quad h \in \Gamma, t \in[0, \alpha], \gamma \in P_{2},
$$

where $z(t, \gamma, h)$ is the solution of the IVP (4.13)-(4.14) for $t \in[0, \alpha], \gamma \in P_{2}$ and $h \in \Gamma$. Moreover, the functions

$$
\mathbb{R} \times \Gamma \supset[0, \alpha] \times P_{2} \rightarrow \mathcal{L}\left(\Gamma, \mathbb{R}^{n}\right), \quad(t, \gamma) \mapsto D_{2} x(t, \gamma)
$$

and

$$
\mathbb{R} \times \Gamma \supset[0, \alpha] \times P_{2} \rightarrow \mathcal{L}(\Gamma, C), \quad(t, \gamma) \mapsto D_{2} x_{t}(\cdot, \gamma)
$$

are continuous.

Proof Let $\gamma=(\varphi, \theta, \xi) \in P_{2}$ be fixed, and let $h_{k}=\left(h_{k}^{\varphi}, h_{k}^{\theta}, h_{k}^{\xi}\right) \in \Gamma(k \in \mathbb{N})$ be a sequence with $\left|h_{k}\right|_{\Gamma} \rightarrow 0$ as $k \rightarrow \infty$ and $\gamma+h_{k} \in P$ for $k \in \mathbb{N}$. To simplify notation, let $x^{k}(t):=x\left(t, \gamma+h_{k}\right)$, $x(t):=x(t, \gamma), u(s):=s-\tau\left(s, x_{s}, \xi\right), u^{k}(s):=s-\tau\left(s, x_{s}^{k}, \xi+h_{k}^{\xi}\right)$ and $z^{h_{k}}(t):=z\left(t, \gamma, h_{k}\right)$. Then

$$
\begin{aligned}
x^{k}(t) & =\varphi(0)+h_{k}^{\varphi}(0)+\int_{0}^{t} f\left(s, x_{s}^{k}, x^{k}\left(u^{k}(s)\right), \theta+h_{k}^{\theta}\right) d s, \quad t \in[0, \alpha], \\
x(t) & =\varphi(0)+\int_{0}^{t} f\left(s, x_{s}, x(u(s)), \theta\right) d s, \quad t \in[0, \alpha],
\end{aligned}
$$

and

$$
z^{h_{k}}(t)=h_{k}^{\varphi}(0)+\int_{0}^{t} L(s, x)\left(z_{s}^{h_{k}}, h_{k}^{\theta}, h_{k}^{\xi}\right) d s, \quad t \in[0, \alpha]
$$

We have

$$
\begin{aligned}
x^{k}(t)-x(t)-z^{h_{k}}(t)=\int_{0}^{t}( & f\left(s, x_{s}^{k}, x^{k}\left(u^{k}(s)\right), \theta+h_{k}^{\theta}\right)-f\left(s, x_{s}, x(u(s)), \theta\right) \\
& \left.-L(s, x)\left(z_{s}^{h_{k}}, h_{k}^{\theta}, h_{k}^{\xi}\right)\right) d s
\end{aligned}
$$


The definitions of $\omega_{f}$ and $L(s, x)$ (see (4.3) and (4.10), respectively) yield for $s \in[0, \alpha]$

$$
\begin{aligned}
& f\left(s, x_{s}^{k}, x^{k}\left(u^{k}(s)\right), \theta+h_{k}^{\theta}\right)-f\left(s, x_{s}, x(u(s)), \theta\right)-L(s, x)\left(z_{s}^{h_{k}}, h_{k}^{\theta}, h_{k}^{\xi}\right) \\
& =\quad D_{2} f\left(s, x_{s}, x(u(s)), \theta\right)\left(x_{s}^{k}-x_{s}-z_{s}^{h_{k}}\right)+D_{3} f\left(s, x_{s}, x(u(s)), \theta\right)\left(x^{k}\left(u^{k}(s)\right)-x(u(s))\right) \\
& \quad+D_{3} f\left(s, x_{s}, x(u(s)), \theta\right)\left(\dot{x}(u(s))\left(D_{2} \tau\left(s, x_{s}, \xi\right) z_{s}^{h_{k}}+D_{3} \tau\left(s, x_{s}, \xi\right) h_{k}^{\xi}\right)-z^{h_{k}}(u(s))\right) \\
& \quad+\omega_{f}\left(s, x_{s}, x\left(u(s), \theta, x_{s}^{k}, x^{k}\left(u^{k}(s)\right), \theta+h_{k}^{\theta}\right) .\right.
\end{aligned}
$$

Relation (4.4) and simple manipulations give

$$
\begin{aligned}
x^{k}\left(u^{k}(s)\right)-x(u(s))+\dot{x}(u(s))\left(D_{2} \tau\left(s, x_{s}, \xi\right) z_{s}^{h_{k}}+D_{3} \tau\left(s, x_{s}, \xi\right) h_{k}^{\xi}\right)-z^{h_{k}}(u(s)) \\
=\quad x^{k}\left(u^{k}(s)\right)-x\left(u^{k}(s)\right)-z^{h_{k}}\left(u^{k}(s)\right)+x\left(u^{k}(s)\right)-x(u(s))-\dot{x}(u(s))\left(u^{k}(s)-u(s)\right) \\
\quad-\dot{x}(u(s)) \omega_{\tau}\left(s, x_{s}, \xi, x_{s}^{k}, \xi+h_{k}^{\xi}\right)-\dot{x}(u(s)) D_{2} \tau\left(s, x_{s}, \xi\right)\left(x_{s}^{k}-x_{s}-z_{s}^{h_{k}}\right) \\
\quad+z^{h_{k}}\left(u^{k}(s)\right)-z^{h_{k}}(u(s)) .
\end{aligned}
$$

Relation (3.4) and (4.16) imply

$$
\left|z^{h_{k}}\left(u^{k}(s)\right)-z^{h_{k}}(u(s))\right| \leq N_{2}\left|h_{k}\right|_{\Gamma}\left|u^{k}(s)-u(s)\right| \leq N_{2} K_{0}\left|h_{k}\right|_{\Gamma}^{2} .
$$

Using (3.1), (A1) (ii), (A2) (ii), and combining (4.29), (4.30), (4.31) and (4.32) we get

$$
\begin{aligned}
&\left|x^{k}(t)-x(t)-z^{h_{k}}(t)\right| \\
& \leq \int_{0}^{t}[ L_{1}\left(\left|x_{s}^{k}-x_{s}-z_{s}^{h_{k}}\right|_{C}+\left|x^{k}\left(u^{k}(s)\right)-x\left(u^{k}(s)\right)-z^{h_{k}}\left(u^{k}(s)\right)\right|\right. \\
&+\left|x\left(u^{k}(s)\right)-x(u(s))-\dot{x}(u(s))\left(u^{k}(s)-u(s)\right)\right| \\
&\left.+N\left|\omega_{\tau}\left(s, x_{s}, \xi, x_{s}^{k}, \xi+h_{k}^{\xi}\right)\right|+N L_{2}\left|x_{s}^{k}-x_{s}-z_{s}^{h_{k}}\right|_{C}+N_{2} K_{0}\left|h_{k}\right|_{\Gamma}^{2}\right) \\
&\left.+\left|\omega_{f}\left(s, x_{s}, x(u(s)), \theta, x_{s}^{k}, x^{k}\left(u^{k}(s)\right), \theta+h_{k}^{\theta}\right)\right|\right] d s, \quad t \in[0, \alpha] .
\end{aligned}
$$

Let $N_{0}$ be defined by (4.12). Then

$$
\left|x^{k}(t)-x(t)-z^{h_{k}}(t)\right| \leq a_{k}+b_{k}+c_{k}+d_{k}+L_{1} N_{0} \int_{0}^{t}\left|x_{s}^{k}-x_{s}-z_{s}^{h_{k}}\right|_{C} d s, \quad t \in[0, \alpha],
$$

where

$$
\begin{aligned}
a_{k} & :=\int_{0}^{\alpha}\left|\omega_{f}\left(s, x_{s}, x(u(s)), \theta, x_{s}^{k}, x^{k}\left(u^{k}(s)\right), \theta+h_{k}^{\theta}\right)\right| d s, \\
b_{k} & :=L_{1} N \int_{0}^{\alpha}\left|\omega_{\tau}\left(s, x_{s}, \xi, s, x_{s}^{k}, \xi+h_{k}^{\xi}\right)\right| d s, \\
c_{k} & :=L_{1} \int_{0}^{\alpha}\left|x\left(u^{k}(s)\right)-x(u(s))-\dot{x}(u(s))\left(u^{k}(s)-u(s)\right)\right| d s,
\end{aligned}
$$

and

$$
d_{k}:=\alpha N_{2} K_{0}\left|h_{k}\right|_{\Gamma}^{2}
$$

Since $\left|x_{0}^{k}-x_{0}-z_{0}\right|_{C}=0$, Lemma 2.1 is applicable for (4.34), and it yields

$$
\left|x^{k}(t)-x(t)-z^{h_{k}}(t)\right| \leq\left|x_{t}^{k}-x_{t}-z_{t}\right|_{C} \leq\left(a_{k}+b_{k}+c_{k}+d_{k}\right) N_{1}, \quad t \in[0, \alpha],
$$


where $N_{1}:=e^{L_{1} N_{0} \alpha}$, and hence

$$
\frac{\left|x^{k}(t)-x(t)-z^{h_{k}}(t)\right|}{\left|h_{k}\right|_{\Gamma}} \leq \frac{\left|x_{t}^{k}-x_{t}-z_{t}^{h_{k}}\right|_{C}}{\left|h_{k}\right|_{\Gamma}} \leq \frac{a_{k}+b_{k}+c_{k}+d_{k}}{\left|h_{k}\right|_{\Gamma}} N_{1}, \quad t \in[0, \alpha],
$$

which proves both (4.27) and (4.28), since Lemmas 4.1, 4.2 and (4.38) show that

$$
\lim _{k \rightarrow \infty} \frac{a_{k}+b_{k}+c_{k}+d_{k}}{\left|h_{k}\right|_{\Gamma}}=0
$$

The continuity of $D_{2} x(t, \gamma)$ follows from Lemma 4.5 .

Remark 4.8 We comment that if in the statement of Theorem 4.7 the set $P_{2}$ is replaced by $P_{1}$, the statements are valid without assumptions (A2) (iv) and (v). To see this we refer to Remark 4.6, and in the proof of Theorem 4.7 we use Lemma 4.1 of [12] to show that $c_{k} /\left|h_{k}\right|_{\Gamma} \rightarrow 0$ as $k \rightarrow \infty$. We also note that continuous differentiability of $x$ wrt the parameters holds in a neighborhood of $\gamma$, since $P_{1}$ is open in $P$. See Theorem 4.7 in [12] for a related result.

\section{Second-order differentiability wrt the parameters}

To obtain second-order differentiability wrt the parameters we need more smoothness of the initial functions. Therefore we introduce the parameter set

$$
\Gamma_{2}:=W^{2, \infty} \times \Theta \times \Xi
$$

equipped with the norm $|h|_{\Gamma_{2}}:=\left|h^{\varphi}\right|_{W^{2, \infty}}+\left|h^{\theta}\right|_{\Theta}+\left|h^{\xi}\right|_{\Xi}$. We will show in Theorem 5.17 below that the parameter map

$$
\Gamma_{2} \supset\left(P_{2} \cap \Gamma_{2}\right) \rightarrow \mathbb{R}^{n}, \quad \gamma \rightarrow x(t, \gamma)
$$

is twice differentiable at every point $\gamma \in P_{2} \cap \Gamma_{2} \cap \mathcal{P}$. The proof will be based on a sequence of lemmas.

We assume throughout this section

(H) $\gamma=(\varphi, \theta, \xi) \in P_{2} \cap \Gamma_{2}, h=\left(h^{\varphi}, h^{\theta}, h^{\xi}\right) \in \Gamma, h_{k}=\left(h_{k}^{\varphi}, h_{k}^{\theta}, h_{k}^{\xi}\right) \in \Gamma(k \in \mathbb{N})$ are so that $\left|h_{k}\right|_{\Gamma} \rightarrow 0$ as $k \rightarrow \infty, \gamma+h_{k} \in P_{2}$ for $k \in \mathbb{N}$, and $\left|h_{k}\right|_{\Gamma} \neq 0$ for $k \in \mathbb{N}$. Let $x^{k}(t):=x\left(t, \gamma+h_{k}\right)$ and $x(t):=x(t, \gamma)$ be the solutions of the IVP (1.1)-(1.2), $z^{k, h}(t):=$ $D_{2} x\left(t, \gamma+h_{k}\right) h$ and $z^{h}(t):=D_{2} x(t, \gamma) h$ be the solutions of the IVP (4.13)-(4.14). 
The simplifying notations for $t \in[0, \alpha]$ and $k \in \mathbb{N}$

$$
\begin{aligned}
u(t) & :=t-\tau\left(t, x_{t}, \xi\right) \\
u^{k}(t) & :=t-\tau\left(t, x_{t}^{k}, \xi+h_{k}^{\xi}\right), \\
\mathbf{v}(t) & :=\left(t, x_{t}, x(u(t)), \theta\right) \\
\mathbf{v}^{k}(t) & :=\left(t, x_{t}^{k}, x^{k}\left(u^{k}(t)\right), \theta\right), \\
A\left(t, h^{\varphi}, h^{\xi}\right) & :=D_{2} \tau\left(t, x_{t}, \xi\right) h^{\varphi}+D_{3} \tau\left(t, x_{t}, \xi\right) h^{\xi}, \\
A^{k}\left(t, h^{\varphi}, h^{\xi}\right) & :=D_{2} \tau\left(t, x_{t}^{k}, \xi+h_{k}^{\xi}\right) h^{\varphi}+D_{3} \tau\left(t, x_{t}^{k}, \xi+h_{k}^{\xi}\right) h^{\xi}, \\
E\left(t, h^{\varphi}, h^{\xi}\right) & :=-\dot{x}(u(t)) A\left(t, h^{\varphi}, h^{\xi}\right)+h^{\varphi}\left(-\tau\left(t, x_{t}, \xi\right)\right), \quad \text { a.e. } t \in[0, \alpha], \\
E^{k}\left(t, h^{\varphi}, h^{\xi}\right) & :=-\dot{x}^{k}\left(u^{k}(t)\right) A^{k}\left(t, h^{\varphi}, h^{\xi}\right)+h^{\varphi}\left(-\tau\left(t, x_{t}^{k}, \xi+h_{k}^{\xi}\right)\right), \quad \text { a.e. } t \in[0, \alpha], \\
F\left(t, h^{\varphi}, h^{\xi}\right) & :=-\ddot{x}(u(t)) A\left(t, h^{\varphi}, h^{\xi}\right)+\dot{h}^{\varphi}\left(-\tau\left(t, x_{t}, \xi\right)\right), \quad \text { a.e. } t \in[0, \alpha], \\
F^{k}\left(t, h^{\varphi}, h^{\xi}\right) & :=-\ddot{x}^{k}\left(u^{k}(t)\right) A^{k}\left(t, h^{\varphi}, h^{\xi}\right)+\dot{h}^{\varphi}\left(-\tau\left(t, x_{t}^{k}, \xi+h_{k}^{\xi}\right)\right), \quad \text { a.e. } t \in[0, \alpha]
\end{aligned}
$$

will be used throughout this section. For simplicity of the notation we define $h_{0}:=0=(0,0,0) \in$ $\Gamma$, and accordingly, $x^{0}:=x, u^{0}:=u, z^{0, h}:=z^{h}, A^{0}:=A, E^{0}:=E$. Note that in all the above abbreviations the dependence on $\gamma$ is omitted from the notation but it should be kept in mind. With these notations the operator $L(t, x)$ defined by (4.10) can be written shortly as

$$
L(t, x) h=D_{2} f(\mathbf{v}(t)) h^{\varphi}+D_{3} f(\mathbf{v}(t)) E\left(t, h^{\varphi}, h^{\xi}\right)+D_{4} f(\mathbf{v}(t)) h^{\theta} .
$$

Lemma 5.1 Assume (A1) (i)-(iv), (A2) (i)-(iv) and $\gamma=(\varphi, \theta, \xi) \in P$ is such that $\varphi \in W^{2, \infty}$. Then there exists $K_{4}=K_{4}(\gamma) \geq 0$ such that the solution $x(t)=x(t, \gamma)$ of the IVP (1.1)-(1.2) satisfies

$$
|\dot{x}(t)-\dot{x}(\bar{t})| \leq K_{4}|t-\bar{t}| \quad \text { for } t, \bar{t} \in[-r, 0) \quad \text { and } \quad t, \bar{t} \in(0, \alpha] .
$$

Moreover, if in addition $\gamma \in \mathcal{P}$, then $x \in W^{2, \infty}\left([-r, \alpha], \mathbb{R}^{n}\right)$, and

$$
|\dot{x}(t)-\dot{x}(\bar{t})| \leq K_{4}|t-\bar{t}| \quad \text { for } t, \bar{t} \in[-r, \alpha] .
$$

Proof The Mean Value Theorem and the definition of the $W^{2, \infty}$-norm yield

$$
|\dot{x}(t)-\dot{x}(\bar{t})|=|\dot{\varphi}(t)-\dot{\varphi}(\bar{t})| \leq|\varphi|_{W^{2, \infty}}|t-\bar{t}|, \quad t, \bar{t} \in[-r, 0) .
$$

For $t, \bar{t} \in(0, \alpha]$ it follows from (A1) (ii), (iv), (A2) (ii), (iv), (3.1) and (3.6) with $k=0$

$$
\begin{aligned}
|\dot{x}(t)-\dot{x}(\bar{t})| & =\left|f\left(t, x_{t}, x(u(t)), \theta\right)-f\left(\bar{t}, x_{\bar{t}}, x(u(\bar{t})), \theta\right)\right| \\
& \leq L_{1}\left(|t-\bar{t}|+\left|x_{t}-x_{\bar{t}}\right|_{C}+|x(u(t))-x(u(\bar{t}))|\right) \\
& \leq L_{1}\left(1+N+N L_{2}(1+N)\right)|t-\bar{t}| .
\end{aligned}
$$

Hence (5.1) is satisfied with $K_{4}:=\max \left\{|\varphi|_{W^{2, \infty}}, L_{1}\left[1+N+N L_{2}(1+N)\right]\right\}$.

If $\gamma \in \mathcal{P}$, then $\dot{x}$ is continuous, and (5.1) yields that it is Lipschitz continuous on $[-r, \alpha]$ with the Lipschitz constant $K_{4}$, so, in particular, $x \in W^{2, \infty}\left([-r, \alpha], \mathbb{R}^{n}\right)$. 
Lemma 5.2 Assume (A1) (i)-(iii), (A2) (i)-(v), and (H). Then

$$
\lim _{k \rightarrow \infty} \frac{1}{\left|h_{k}\right|_{\Gamma}} \int_{0}^{\alpha}\left|\dot{x}^{k}(s)-\dot{x}(s)-\dot{z}^{h_{k}}(s)\right| d s=0,
$$

and

$$
\lim _{k \rightarrow \infty} \frac{1}{\left|h_{k}\right|_{\Gamma}} \int_{0}^{\alpha}\left|\dot{x}^{k}\left(u^{k}(s)\right)-\dot{x}\left(u^{k}(s)\right)-\dot{z}^{h_{k}}\left(u^{k}(s)\right)\right| d s=0 .
$$

Proof Using (4.29), (4.33), (4.34) and (4.39) we get

$$
\begin{aligned}
& \int_{0}^{\alpha}\left|\dot{x}^{k}(s)-\dot{x}(s)-\dot{z}^{h_{k}}(s)\right| d s \\
& \leq \int_{0}^{\alpha}[ L_{1}\left(\left|x_{s}^{k}-x_{s}-z_{s}^{h_{k}}\right|_{C}+\left|x^{k}\left(u^{k}(s)\right)-x\left(u^{k}(s)\right)-z^{h_{k}}\left(u^{k}(s)\right)\right|\right. \\
& \quad+\left|x\left(u^{k}(s)\right)-x(u(s))-\dot{x}(u(s))\left(u^{k}(s)-u(s)\right)\right| \\
&\left.+N\left|\omega_{\tau}\left(s, x_{s}, \xi, x_{s}^{k}, \xi+h_{k}^{\xi}\right)\right|+N L_{2}\left|x_{s}^{k}-x_{s}-z_{s}^{h_{k}}\right|_{C}+N_{2} K_{0}\left|h_{k}\right|_{\Gamma}^{2}\right) \\
&\left.+\left|\omega_{f}\left(s, x_{s}, x(u(s)), \theta, x_{s}^{k}, x^{k}\left(u^{k}(s)\right), \theta+h_{k}^{\theta}\right)\right|\right] d s \\
& \leq a_{k}+b_{k}+c_{k}+d_{k}+L_{1} N_{0} \int_{0}^{\alpha}\left|x_{s}^{k}-x_{s}-z_{s}^{h_{k}}\right|_{C} d s \\
& \leq\left(a_{k}+b_{k}+c_{k}+d_{k}\right)\left(1+L_{1} N_{0} N_{1} \alpha\right)
\end{aligned}
$$

where $a_{k}, b_{k}, c_{k}$ and $d_{k}$ are defined by (4.35)-(4.38), respectively. Then (5.3) is obtained from (4.41).

Relation (5.4) follows from (5.3), $x^{k}(s)-x(s)-z^{h_{k}}(s)=0$ for $s \in[-r, 0], \mid \dot{x}^{k}(s)-\dot{x}(s)-$ $\left.\dot{z}^{h_{k}}(s)\left|\leq\left(L+N_{2}\right)\right| h_{k}\right|_{\Gamma}$ for $s \in[-r, 0]$, and Lemmas 2.9 and 3.3.

Lemma 5.3 Assume (A1) (i)-(v), (A2) (i)-(vi), (H) and $\gamma \in \mathcal{P}$. Then there exists $N_{4}=$ $N_{4}(\gamma) \geq 0$ such that

$$
\left|\dot{z}^{h}(s)-\dot{z}^{h}(\bar{s})\right| \leq N_{4}|h|_{\Gamma_{2}}|s-\bar{s}|, \quad \text { for } \quad s, \bar{s} \in[-r, 0) \quad \text { and } \quad s, \bar{s} \in(0, \alpha], \quad h \in \Gamma_{2} .
$$

Proof For $h \in \Gamma_{2}$, i.e., $h^{\varphi} \in W^{2, \infty}$, the function $\dot{h}^{\varphi}$ is continuous, and for $s, \bar{s} \in[-r, 0)$

$$
\left|\dot{z}^{h}(s)-\dot{z}^{h}(\bar{s})\right|=\left|\dot{h}^{\varphi}(s)-\dot{h}^{\varphi}(\bar{s})\right| \leq\left|h^{\varphi}\right|_{W^{2, \infty}}|s-\bar{s}| \leq|h|_{\Gamma_{2}}|s-\bar{s}| .
$$

Since $\gamma \in \mathcal{P}, L(s, x)$ is defined and continuous for all $s \in[0, \alpha]$, so $\dot{z}^{h}$ is continuous on $(0, \alpha]$. For $s, \bar{s} \in(0, \alpha](4.11)$ and (4.13) imply

$$
\begin{aligned}
\left|\dot{z}^{h}(s)-\dot{z}^{h}(\bar{s})\right|= & \left|L(s, x)\left(z_{s}^{h}, h^{\theta}, h^{\xi}\right)-L(\bar{s}, x)\left(z_{\bar{s}}^{h}, h^{\theta}, h^{\xi}\right)\right| \\
\leq & \left|[L(s, x)-L(\bar{s}, x)]\left(z_{s}^{h}, h^{\theta}, h^{\xi}\right)\right|+\left|L(\bar{s}, x)\left(z_{s}^{h}-z_{\bar{s}}^{h}, 0,0\right)\right| \\
\leq & \left|\left[D_{2} f(\mathbf{v}(s))-D_{2} f(\mathbf{v}(\bar{s}))\right] z_{s}^{h}\right|+\left|\left[D_{3} f(\mathbf{v}(s))-D_{3} f(\mathbf{v}(\bar{s}))\right] E\left(s, z_{s}^{h}, h^{\xi}\right)\right| \\
& +\left|D_{3} f(\mathbf{v}(\bar{s}))\left[E\left(s, z_{s}^{h}, h^{\xi}\right)-E\left(\bar{s}, z_{\bar{s}}^{h}, h^{\xi}\right)\right]\right| \\
& +\left|\left[D_{4} f(\mathbf{v}(s))-D_{4} f(\mathbf{v}(\bar{s}))\right] h^{\theta}\right|+L_{1} N_{0}\left|z_{s}^{h}-z_{\bar{s}}^{h}\right|_{C} .
\end{aligned}
$$

We have by (3.1) and (3.6) with $k=0$ for $s, \bar{s} \in[0, \alpha]$

$$
|\mathbf{v}(s)-\mathbf{v}(\bar{s})| \leq|s-\bar{s}|+\left|x_{s}-x_{\bar{s}}\right|_{C}+|x(u(s))-x(u(\bar{s}))| \leq K_{5}|s-\bar{s}|
$$


and

$$
\left|\left(s, x_{s}, \xi\right)-\left(\bar{s}, x_{\bar{s}}, \xi\right)\right| \leq(1+N)|s-\bar{s}|
$$

with $K_{5}:=\left(1+N+N L_{2}(1+N)\right)$ and $(1+N):=1+N$. Let $L_{3}:=L_{3}\left(\alpha, M_{1}, M_{2}, M_{3}\right)$ and $L_{5}:=L_{5}\left(\alpha, M_{1}, M_{2}, M_{3}\right)$ be defined by (A1) (v) and (A2) (vi), respectively.

The definition of $A,(\mathrm{~A} 2)$ (ii) and (4.15) give

$$
\left|A\left(s, z_{s}^{h}, h^{\xi}\right)\right| \leq\left|D_{2} \tau\left(s, x_{s}, \xi\right) z_{s}^{h}\right|+\left|D_{3} \tau\left(s, x_{s}, \xi\right) h^{\xi}\right| \leq K_{6}|h|_{\Gamma}, \quad s \in[0, \alpha], h \in \Gamma, \gamma \in P_{2}
$$

with $K_{6}:=L_{2}\left(N_{1}+1\right)$, and by using (A2) (ii), (vi), (4.15), (4.16), (5.8)

$$
\begin{aligned}
\left|A\left(s, z_{s}^{h}, h^{\xi}\right)-A\left(\bar{s}, z_{\bar{s}}^{h}, h^{\xi}\right)\right| \leq & \left|\left[D_{2} \tau\left(s, x_{s}, \xi\right)-D_{2} \tau\left(\bar{s}, x_{\bar{s}}, \xi\right)\right] z_{s}^{h}\right|+\left|D_{2} \tau\left(\bar{s}, x_{\bar{s}}, \xi\right)\left[z_{s}^{h}-z_{\bar{s}}^{h}\right]\right| \\
& +\left|\left[D_{3} \tau\left(s, x_{s}, \xi\right)-D_{3} \tau\left(\bar{s}, x_{\bar{s}}, \xi\right)\right] h^{\xi}\right| \\
\leq & K_{7}|s-\bar{s}||h|_{\Gamma}, \quad s, \bar{s} \in[0, \alpha]
\end{aligned}
$$

with $K_{7}:=L_{5}(1+N) N_{1}+L_{2} N_{2}+L_{5}(1+N)$. Relations (3.1), (4.15) and (5.9) yield

$$
\begin{aligned}
\left|E\left(s, z_{s}^{h}, h^{\xi}\right)\right| & \leq|\dot{x}(u(s))|\left|A\left(s, z_{s}^{h}, h^{\xi}\right)\right|+\left|z^{h}(u(s))\right| \\
& \leq K_{8}|h|_{\Gamma}, \quad s \in[0, \alpha], h \in \Gamma, \gamma \in P_{2}
\end{aligned}
$$

with $K_{8}:=N K_{6}+N_{1}$, and using (3.1), (3.6) with $k=0,(4.16),(5.2),(5.9)$ and (5.10)

$$
\begin{aligned}
&\left|E\left(s, z_{s}^{h}, h^{\xi}\right)-E\left(\bar{s}, z_{\bar{s}}^{h}, h^{\xi}\right)\right| \\
& \leq\left|[\dot{x}(u(s))-\dot{x}(u(\bar{s}))] A\left(s, z_{s}^{h}, h^{\xi}\right)\right|+\left|\dot{x}(u(\bar{s}))\left[A\left(s, z_{s}^{h}, h^{\xi}\right)-A\left(\bar{s}, z_{\bar{s}}^{h}, h^{\xi}\right)\right]\right| \\
&+\left|z^{h}(u(s))-z^{h}(u(\bar{s}))\right| \\
& \leq K_{9}|s-\bar{s}||h|_{\Gamma}, \quad s, \bar{s} \in[0, \alpha]
\end{aligned}
$$

with $K_{9}=K_{9}(\gamma):=K_{4} L_{2}(1+N) K_{6}+N K_{7}+N_{2} L_{2}(1+N)$. Then combining (5.6) with (5.7), (5.11) and (5.12) yields

$$
\left|\dot{z}^{h}(s)-\dot{z}^{h}(\bar{s})\right| \leq\left(L_{3} K_{5} N_{1}+L_{3} K_{5} K_{8}+L_{1} K_{9}+L_{3} K_{5}+L_{1} N_{0} N_{2}\right)|s-\bar{s}||h|_{\Gamma}
$$

for $s, \bar{s} \in[0, \alpha]$ and $h \in \Gamma$. Hence $N_{4}:=\max \left\{1, L_{3} K_{5} N_{1}+L_{3} K_{5} K_{8}+L_{1} K_{9}+L_{3} K_{5}+L_{1} N_{0} N_{2}\right\}$ satisfies (5.5).

Lemma 5.4 Assume (A1) (i)-(v), (A2) (i)-(vi), (H) and $\gamma \in \mathcal{P}$. Then

$$
\lim _{k \rightarrow \infty} \sup _{\substack{h \neq 0 \\ h \in \Gamma_{2}}} \frac{1}{|h|_{\Gamma_{2}}} \int_{0}^{\alpha}\left|\dot{z}^{h}\left(u^{k}(s)\right)-\dot{z}^{h}(u(s))\right| d s=0 .
$$

Proof Since $\gamma \in P_{2}$ and $u(0) \leq 0$, it follows that $u$ has finitely many zeros on $[0, \alpha]$. Let $0 \leq s_{1}<s_{2}<\cdots<s_{\ell} \leq \alpha$ be the mesh points where $u\left(s_{i}\right)=0,0<\varepsilon<\min \left\{s_{i+1}-s_{i}\right.$ : $i=1, \ldots, \ell-1\} / 2$ be fixed, and introduce $s_{i}^{\prime}:=\min \left\{s_{i}+\varepsilon, \alpha\right\}$ and $s_{i}^{\prime \prime}:=\max \left\{s_{i}-\varepsilon, 0\right\}$ for $i=1, \ldots, \ell, s_{0}^{\prime}:=0, s_{\ell+1}^{\prime \prime}:=\alpha$, and let

$$
M:=\min _{i=1, \ldots, \ell-1} \min _{s \in\left[s_{i}^{\prime}, s_{i+1}^{\prime \prime}\right]}|u(s)| .
$$


We have $M>0$. Relation (3.4) yields that there exist $k_{0}>0$ such that $\left|u^{k}-u\right|_{C([0, \alpha], \mathbb{R})}<\frac{M}{2}$ for $k \geq k_{0}$. Then for $k \geq k_{0}$ it follows $\left|u^{k}(s)\right| \geq \frac{M}{2}$ for $s \in\left[s_{i}^{\prime}, s_{i+1}^{\prime \prime}\right]$ and $i=0, \ldots, \ell$. Note that $h \in \Gamma_{2}$ and $\gamma \in \mathcal{P}$ yield $\dot{z}^{h}$ is continuous on $[-r, 0)$ and $(0, \alpha]$, and (4.16) implies $\left|\dot{z}^{h}(s)\right| \leq$ $N_{2}|h|_{\Gamma} \leq N_{2}|h|_{\Gamma_{2}}$ for $s \neq 0$. Therefore $\left|\dot{z}^{h}\left(u^{k}(s)\right)\right| \leq N_{2}|h|_{\Gamma_{2}}$ for a.e. $s \in[0, \alpha]$, since, by assumption (H), $\gamma+h_{k} \in P_{2}$, hence $u^{k} \in \mathcal{P} \mathcal{M}([0, \alpha],[-r, \alpha])$. Then (3.4), (4.16) and (5.5) yield

$$
\begin{aligned}
& \int_{0}^{\alpha}\left|\dot{z}^{h}\left(u^{k}(s)\right)-\dot{z}^{h}(u(s))\right| d s \\
& \quad \leq \sum_{i=1}^{\ell} \int_{s_{i}^{\prime \prime}}^{s_{i}^{\prime}}\left[\left|\dot{z}^{h}\left(u^{k}(s)\right)\right|+\left|\dot{z}^{h}(u(s))\right|\right] d s+\sum_{i=0}^{\ell} \int_{s_{i}^{\prime}}^{s_{i+1}^{\prime \prime}}\left|\dot{z}^{h}\left(u^{k}(s)\right)-\dot{z}^{h}(u(s))\right| d s \\
& \quad \leq 4 \ell \varepsilon N_{2}|h|_{\Gamma_{2}}+(\ell+1) \alpha N_{4} K_{0}|h|_{\Gamma_{2}}\left|h_{k}\right|_{\Gamma} .
\end{aligned}
$$

This concludes the proof of (5.13), since $\varepsilon>0$ can be arbitrary close to 0 .

Lemma 5.5 Assume (A1) (i)-(v), (A2) (i)-(vi), (H) and $\gamma \in \mathcal{P}$. Then

$$
\lim _{k \rightarrow \infty} \sup _{\substack{h \neq 0 \\ h \in \Gamma_{2}}} \frac{1}{|h|_{\Gamma_{2}}\left|h_{k}\right|_{\Gamma}} \int_{0}^{\alpha}\left|z^{h}\left(u^{k}(s)\right)-z^{h}(u(s))-\dot{z}^{h}(u(s))\left(u^{k}(s)-u(s)\right)\right| d s=0 .
$$

Proof Let $s_{i}, s_{i}^{\prime}, s_{i}^{\prime \prime}, \ell, \varepsilon, M$ and $k_{0}$ be defined as in the proof of Lemma 5.4. Then $\mid u(s)+$ $\nu\left(u^{k}(s)-u(s)\right) \mid>\frac{M}{2}$, and $u(s)$ and $u(s)+\nu\left(u^{k}(s)-u(s)\right)$ are both either positive or negative for $s \in\left[s_{i}^{\prime}, s_{i+1}^{\prime \prime}\right], \nu \in[0,1]$ and $i=0, \ldots, \ell$. Therefore (3.4) and (5.5) yield

$$
\left|\dot{z}^{h}\left(u(s)+\nu\left(u^{k}(s)-u(s)\right)\right)-\dot{z}^{h}(u(s))\right| \leq N_{4}|h|_{\Gamma_{2}}\left|u^{k}(s)-u(s)\right| \leq N_{4} K_{0}|h|_{\Gamma_{2}}\left|h_{k}\right|_{\Gamma} .
$$

Hence, using Fubini's Theorem, (3.4) and (4.16) we have

$$
\begin{aligned}
& \int_{0}^{\alpha}\left|z^{h}\left(u^{k}(s)\right)-z^{h}(u(s))-\dot{z}^{h}(u(s))\left(u^{k}(s)-u(s)\right)\right| d s \\
& \left.\leq \sum_{i=1}^{\ell} \int_{s_{i}^{\prime \prime}}^{s_{i}^{\prime}}\left(\left|z^{h}\left(u^{k}(s)\right)-z^{h}(u(s))\right|+\left|\dot{z}^{h}(u(s))\right| \mid u^{k}(s)-u(s)\right) \mid\right) d s \\
& \quad+\sum_{i=0}^{\ell} \int_{s_{i}^{\prime}}^{s_{i+1}^{\prime \prime}}\left|z^{h}\left(u^{k}(s)\right)-z^{h}(u(s))-\dot{z}^{h}(u(s))\left(u^{k}(s)-u(s)\right)\right| d s \\
& \leq \quad 4 \varepsilon \ell N_{2} K_{0}|h|_{\Gamma}\left|h_{k}\right|_{\Gamma} \\
& \quad+\sum_{i=0}^{\ell} \int_{s_{i}^{\prime}}^{s_{i+1}^{\prime \prime}}\left|\int_{0}^{1}\left[\dot{z}^{h}\left(u(s)+\nu\left(u^{k}(s)-u(s)\right)\right)-\dot{z}^{h}(u(s))\right]\left[u^{k}(s)-u(s)\right] d \nu\right| d s \\
& \leq \quad 4 \varepsilon \ell N_{2} K_{0}|h|_{\Gamma}\left|h_{k}\right|_{\Gamma} \\
& \quad+K_{0}\left|h_{k}\right|_{\Gamma} \sum_{i=0}^{\ell} \int_{0}^{1} \int_{s_{i}^{\prime}}^{s_{i+1}^{\prime \prime}}\left|\dot{z}^{h}\left(u(s)+\nu\left(u^{k}(s)-u(s)\right)\right)-\dot{z}^{h}(u(s))\right| d s d \nu \\
& \leq \quad 4 \varepsilon \ell N_{2} K_{0}|h|_{\Gamma_{2}}\left|h_{k}\right|_{\Gamma}+K_{0}^{2}(\ell+1) \alpha N_{4}|h|_{\Gamma_{2}}\left|h_{k}\right|_{\Gamma}^{2} .
\end{aligned}
$$

This completes the proof of (5.14), since $\varepsilon>0$ is arbitrary close to 0 . 
Lemma 5.6 Assume (A1) (i)-(iii), (A2) (i)-(v), (H). Then

$$
\lim _{k \rightarrow \infty} \sup _{\substack{h \neq 0 \\ h \in \Gamma}} \frac{1}{|h|_{\Gamma}} \int_{0}^{\alpha}\left|\dot{z}^{k, h}(s)-\dot{z}^{h}(s)\right| d s=0
$$

and

$$
\lim _{k \rightarrow \infty} \sup _{|h|_{\Gamma} \neq 0} \frac{1}{|h|_{\Gamma}\left|h_{k}\right|_{\Gamma}} \int_{0}^{\alpha}\left|z^{k, h}\left(u^{k}(s)\right)-z^{h}\left(u^{k}(s)\right)-\left[z^{k, h}(u(s))-z^{h}(u(s))\right]\right| d s=0 .
$$

Proof For $s \in[0, \alpha]$ combining (4.11), (4.13), (4.17), (4.22) and (4.25) we get

$$
\begin{aligned}
\mid \dot{z}^{k, h}(s) & -\dot{z}^{h}(s) \mid \\
& \leq\left|L\left(s, x^{k}\right)\left(z_{s}^{k, h}-z_{s}^{h}, 0,0\right)\right|+\left|\left(L\left(s, x^{k}\right)-L(s, x)\right)\left(z_{s}^{h}, h^{\theta}, h^{\xi}\right)\right| \\
& \leq L_{1} N_{0} c_{1, k} N_{1}|h|_{\Gamma}+c_{0, k}\left(N_{2}+1\right)|h|_{\Gamma}+L_{1} L_{2}\left(N_{2}+1\right)\left|\dot{x}\left(u^{k}(s)\right)-\dot{x}(u(s))\right||h|_{\Gamma} .
\end{aligned}
$$

Hence Lemmas 2.8 and 3.3 yield (5.15).

Define the functions

$$
f^{k, h}(s):=\frac{\left|\dot{z}^{k, h}(s)-\dot{z}^{h}(s)\right|}{|h|_{\Gamma}}
$$

and the set $H:=\{h \in \Gamma: h \neq 0\}$. Note that (4.11), (4.13) and (4.15) yield $\left|\dot{z}^{k, h}(s)\right|=$ $\left|L\left(s, x^{k}\right) z_{s}^{k, h}\right| \leq L_{1} N_{0} N_{1}|h|_{\Gamma}$ for $k \in \mathbb{N}_{0}$ and $s \in[0, \alpha]$, so $\left|f^{k, h}(s)\right| \leq 2 L_{1} N_{0} N_{1}$ for a.e. $s \in[-r, \alpha]$, $k \in \mathbb{N}$ and $h \in H$. Then it follows from (5.15), $z^{k, h}(s)-z^{h}(s)=0$ for $s \in[-r, 0]$, and Lemmas 2.9 and 3.3 that for any fixed $\nu \in[0,1]$

$$
\lim _{k \rightarrow \infty} \sup _{\substack{h \neq 0 \\ h \in \Gamma}} \frac{1}{|h|_{\Gamma}} \int_{0}^{\alpha}\left|\dot{z}^{k, h}\left(u(s)+\nu\left(u^{k}(s)-u(s)\right)\right)-\dot{z}^{h}\left(u(s)+\nu\left(u^{k}(s)-u(s)\right)\right)\right| d s=0 .
$$

(3.4) and Fubini's Theorem yield

$$
\begin{aligned}
& \int_{0}^{\alpha}\left|z^{k, h}\left(u^{k}(s)\right)-z^{h}\left(u^{k}(s)\right)-\left[z^{k, h}(u(s))-z^{h}(u(s))\right]\right| d s \\
& =\int_{0}^{\alpha} \mid \int_{0}^{1}\left[\dot{z}^{k, h}\left(u(s)+\nu\left(u^{k}(s)-u(s)\right)\right)-\dot{z}^{h}\left(u(s)+\nu\left(u^{k}(s)-u(s)\right)\right)\right] \\
& \quad \times\left[u^{k}(s)-u(s)\right] d \nu \mid d s \\
& \leq K_{0}\left|h_{k}\right|_{\Gamma} \int_{0}^{1} \int_{0}^{\alpha}\left|\dot{z}^{k, h}\left(u(s)+\nu\left(u^{k}(s)-u(s)\right)\right)-\dot{z}^{h}\left(u(s)+\nu\left(u^{k}(s)-u(s)\right)\right)\right| d s d \nu
\end{aligned}
$$

Therefore (5.17) and the Dominated Convergence Theorem imply (5.16).

Introduce the notation

$$
p^{k}(t):=x^{k}(t)-x(t)-z^{h_{k}}(t) .
$$

Then, under the assumptions of Theorem 4.7, (4.40) and (4.41) give

$$
\lim _{k \rightarrow \infty} \max _{s \in[-r, \alpha]} \frac{\left|p^{k}(s)\right|}{\left|h_{k}\right|_{\Gamma}}=0 .
$$

To linearize equation (4.13) around a fixed solution $z$ we will need the following results. 
Lemma 5.7 Assume (A1) (i)-(v), (A2) (i)-(vi), (H) and $\gamma \in \mathcal{P}$. Then

(i)

$$
u^{k}(s)-u(s)+A\left(s, z_{s}^{h_{k}}, h_{k}^{\xi}\right)=g_{0}^{k}(s), \quad s \in[0, \alpha]
$$

where

$$
g_{0}^{k}(s):=-\omega_{\tau}\left(s, x_{s}, \xi, x_{s}^{k}, \xi+h_{k}^{\xi}\right)-D_{2} \tau\left(s, x_{s}, \xi\right) p_{s}^{k}
$$

satisfies

$$
\lim _{k \rightarrow \infty} \frac{1}{\left|h_{k}\right|_{\Gamma}} \int_{0}^{\alpha}\left|g_{0}^{k}(s)\right| d s=0
$$

(ii)

$$
x^{k}\left(u^{k}(s)\right)-x(u(s))-E\left(s, z_{s}^{h_{k}}, h_{k}^{\xi}\right)=g_{1}^{k}(s), \quad s \in[0, \alpha]
$$

where

$$
\begin{aligned}
g_{1}^{k}(s):= & p^{k}\left(u^{k}(s)\right)+x\left(u^{k}(s)\right)-x(u(s))-\dot{x}(u(s))\left(u^{k}(s)-u(s)\right)+\dot{x}(u(s)) g_{0}^{k}(s) \\
& +z^{h_{k}}\left(u^{k}(s)\right)-z^{h_{k}}(u(s))
\end{aligned}
$$

satisfies

$$
\lim _{k \rightarrow \infty} \frac{1}{\left|h_{k}\right|_{\Gamma}} \int_{0}^{\alpha}\left|g_{1}^{k}(s)\right| d s=0
$$

and

(iii) if $h_{k} \in \Gamma_{2}$ for $k \in \mathbb{N}$, then

$$
\dot{x}^{k}\left(u^{k}(s)\right)-\dot{x}(u(s))-F\left(s, z_{s}^{h_{k}}, h_{k}^{\xi}\right)=g_{2}^{k}(s), \quad s \in[0, \alpha],
$$

where

$$
\begin{aligned}
g_{2}^{k}(s):= & \dot{x}^{k}\left(u^{k}(s)\right)-\dot{x}\left(u^{k}(s)\right)-\dot{z}^{h_{k}}\left(u^{k}(s)\right)+\dot{z}^{h_{k}}\left(u^{k}(s)\right)-\dot{z}^{h_{k}}(u(s)) \\
& +\dot{x}\left(u^{k}(s)\right)-\dot{x}(u(s))-\ddot{x}(u(s))\left(u^{k}(s)-u(s)\right) \\
& -\ddot{x}(u(s)) \omega_{\tau}\left(s, x_{s}, \xi, x_{s}^{k}, \xi+h_{k}^{\xi}\right)-\ddot{x}(u(s)) D_{2} \tau\left(s, x_{s}, \xi\right) p_{s}^{k}
\end{aligned}
$$

satisfies

$$
\lim _{k \rightarrow \infty} \frac{1}{\left|h_{k}\right|_{\Gamma_{2}}} \int_{0}^{\alpha}\left|g_{2}^{k}(s)\right| d s=0 .
$$

Proof The definition of $\omega_{\tau}$ and $A$ imply

$$
\begin{aligned}
u^{k}(s)-u(s) & +A\left(s, z_{s}^{h_{k}}, h_{k}^{\xi}\right) \\
= & -\left[\tau\left(s, x_{s}^{k}, \xi+h_{k}^{\xi}\right)-\tau\left(s, x_{s}, \xi\right)-D_{2} \tau\left(s, x_{s}, \xi\right)\left(x_{s}^{k}-x_{s}\right)-D_{2} \tau\left(s, x_{s}, \xi\right) h_{k}^{\xi}\right] \\
& -D_{2} \tau\left(s, x_{s}, \xi\right)\left(x_{s}^{k}-x_{s}-z_{s}^{h_{k}}\right), \quad s \in[0, \alpha],
\end{aligned}
$$

which shows (5.19). (5.20) follows from $\left|D_{2} \tau\left(s, x_{s}, \xi\right)\right|_{\mathcal{L}(C, \mathbb{R})} \leq L_{2}$ for $s \in[0, \alpha]$, (4.8) and (5.18).

Relation (4.31) and the definition of $g_{1}^{k}$ yield (5.21). We have by (3.1) and (4.32)

$$
\begin{aligned}
\int_{0}^{\alpha}\left|g_{1}^{k}(s)\right| d s \leq & \alpha \max _{s \in[-r, \alpha]}\left|p^{k}(s)\right|+\int_{0}^{\alpha}\left|x\left(u^{k}(s)\right)-x(u(s))-\dot{x}(u(s))\left(u^{k}(s)-u(s)\right)\right| d s \\
& +N \int_{0}^{\alpha}\left|g_{0}^{k}(s)\right| d s+\alpha N_{2} K_{0}\left|h_{k}\right|_{\Gamma}^{2} .
\end{aligned}
$$


Therefore (5.18), (5.20), and Lemmas 4.1 and 3.3 yield (5.22).

Simple computation and the definition of $g_{2}^{k}$ imply (5.23) immediately. Note that $\gamma \in \mathcal{P}$ yields that $\dot{x}$ is continuous on $[-r, \alpha]$, and $\varphi \in W^{2, \infty}$ and Lemma 5.1 imply that $x \in W^{2, \infty}\left([-r, \alpha], \mathbb{R}^{n}\right)$. Then (3.5) and Lemma 4.1 with $y=\dot{x}$ yield

$$
\lim _{k \rightarrow \infty} \frac{1}{\left|h_{k}\right|_{\Gamma}} \int_{0}^{\alpha}\left|\dot{x}\left(u^{k}(s)\right)-\dot{x}(u(s))-\ddot{x}(u(s))\left(u^{k}(s)-u(s)\right)\right| d s=0 .
$$

We have by (5.1) and Lemma 2.7 that $|\ddot{x}(u(s))| \leq K_{4}$ for a.e. $s \in[0, \alpha]$, therefore

$$
\begin{aligned}
\int_{0}^{\alpha}\left|g_{2}^{k}(s)\right| d s \leq & \int_{0}^{\alpha}\left|\dot{x}^{k}\left(u^{k}(s)\right)-\dot{x}\left(u^{k}(s)\right)-\dot{z}^{h_{k}}\left(u^{k}(s)\right)\right| d s \\
& +\int_{0}^{\alpha}\left|\dot{z}^{h_{k}}\left(u^{k}(s)\right)-\dot{z}^{h_{k}}(u(s))\right| d s \\
& +\int_{0}^{\alpha}\left|\dot{x}\left(u^{k}(s)\right)-\dot{x}(u(s))-\ddot{x}(u(s))\left(u^{k}(s)-u(s)\right)\right| d s \\
& +K_{4} \int_{0}^{\alpha}\left|\omega_{\tau}\left(s, x_{s}, \xi, x_{s}^{k}, \xi+h_{k}^{\xi}\right)\right| d s+\alpha K_{4} L_{2} \max _{s \in[0, \alpha]}\left|p_{s}^{k}\right|_{C} .
\end{aligned}
$$

Hence (4.8), (5.4), (5.13), (5.18) and (5.25) imply (5.24).

We define the notations

$$
\begin{aligned}
& \omega_{D_{2} \tau}(s, \bar{\varphi}, \bar{\xi}, \varphi, \xi, \psi) \\
& \quad:=D_{2} \tau(s, \varphi, \xi) \psi-D_{2} \tau(s, \bar{\varphi}, \bar{\xi}) \psi-D_{22} \tau(s, \bar{\varphi}, \bar{\xi})\langle\psi, \varphi-\bar{\varphi}\rangle-D_{23} \tau(s, \bar{\varphi}, \bar{\xi})\langle\psi, \xi-\bar{\xi}\rangle \\
& \omega_{D_{3} \tau}(s, \bar{\varphi}, \bar{\xi}, \varphi, \xi, \chi) \\
& \quad:=D_{3} \tau(s, \varphi, \xi) \chi-D_{3} \tau(s, \bar{\varphi}, \bar{\xi}) \chi-D_{32} \tau(s, \bar{\varphi}, \bar{\xi})\langle\chi, \varphi-\bar{\varphi}\rangle-D_{33} \tau(s, \bar{\varphi}, \bar{\xi})\langle\chi, \xi-\bar{\xi}\rangle
\end{aligned}
$$

for $s \in[0, \alpha], \bar{\varphi}, \varphi \in \Omega_{1}, \bar{\xi}, \xi \in \Omega_{4}, \psi \in C$ and $\chi \in \Xi$.

Lemma 5.8 Assume (A2) (i)-(vii) and (H). Then

$$
\lim _{k \rightarrow \infty} \sup _{\substack{h \neq 0 \\ h \in \Gamma}} \frac{1}{|h|_{\Gamma}\left|h_{k}\right|_{\Gamma}} \int_{0}^{\alpha}\left|\omega_{D_{2} \tau}\left(s, x_{s}, \xi, x_{s}^{k}, \xi+h_{k}^{\xi}, z_{s}^{k, h}\right)\right| d s=0,
$$

and

$$
\lim _{k \rightarrow \infty} \sup _{\substack{h \neq 0 \\ h \in \Gamma}} \frac{1}{|h|_{\Gamma}\left|h_{k}\right|_{\Gamma}} \int_{0}^{\alpha}\left|\omega_{D_{3} \tau}\left(s, x_{s}, \xi, x_{s}^{k}, \xi+h_{k}^{\xi}, h^{\xi}\right)\right| d s=0 .
$$

Proof Let $L_{5}=L_{5}\left(\alpha, M_{1}, M_{3}\right)$ be defined by (A2) (vi). Then (A2) (vi), (3.2), (4.15) and (4.20) yield for $s \in[0, \alpha]$

$$
\begin{aligned}
\left|D_{2} \tau\left(s, x_{s}^{k}, \xi+h_{k}^{\xi}\right) z_{s}^{k, h}-D_{2} \tau\left(s, x_{s}, \xi\right) z_{s}^{k, h}\right| & \leq L_{5}(L+1) N_{1}\left|h_{k}\right|_{\Gamma}|h|_{\Gamma} \\
\mid D_{22} \tau\left(s, x_{s}, \xi\right)\left\langle z_{s}^{k, h}, x_{s}^{k}-x_{s}\right\rangle & \leq\left. L_{5} N_{1} L|h|_{\Gamma} h_{k}\right|_{\Gamma} \\
\mid D_{23} \tau\left(s, x_{s}, \xi\right)\left\langle z_{s}^{k, h}, h_{k}^{\xi}\right\rangle & \leq L_{5} N_{1}|h|_{\Gamma}\left|h_{k}\right|_{\Gamma}
\end{aligned}
$$

and hence,

$$
\left|\omega_{D_{2} \tau}\left(s, x_{s}, \xi, x_{s}^{k}, \xi+h_{k}^{\xi}, z_{s}^{k, h}\right)\right| \leq 2 L_{5}(L+1) N_{1}\left|h_{k}\right|_{\Gamma}|h|_{\Gamma}, \quad s \in[0, \alpha] .
$$


On the other hand, for $s \in[0, \alpha], k \in \mathbb{N}$ and $0 \neq h \in \Gamma$ such that $\left|x_{s}^{k}-x_{s}\right|_{C}+\left|h_{k}^{\xi}\right|_{\Gamma} \neq 0$ and $\left|z_{s}^{k, h}\right|_{C} \neq 0$, assumption (A2) (vii), (3.2) and (4.15) yield

$$
\begin{aligned}
\sup _{|h|_{\Gamma} \neq 0} & \frac{\left|\omega_{D_{2} \tau}\left(s, x_{s}, \xi, x_{s}^{k}, \xi+h_{k}^{\xi}, z_{s}^{k, h}\right)\right|}{|h|_{\Gamma}\left|h_{k}\right|_{\Gamma}} \\
& =\sup _{|h|_{\Gamma} \neq 0} \frac{\left|\omega_{D_{2} \tau}\left(s, x_{s}, \xi, x_{s}^{k}, \xi+h_{k}^{\xi}, z_{s}^{k, h}\right)\right|}{\left(\left|x_{s}^{k}-x_{s}\right|_{C}+\left|h_{k}^{\xi}\right|_{\Gamma}\right)\left|z_{s}^{k, h}\right|_{C}} \cdot \frac{\left(\left|x_{s}^{k}-x_{s}\right|_{C}+\left|h_{k}^{\xi}\right|_{\Gamma}\right)\left|z_{s}^{k, h}\right|_{C}}{|h|_{\Gamma}\left|h_{k}\right|_{\Gamma}} \\
& \leq(L+1) N_{1} \sup _{|h|_{\Gamma} \neq 0} \frac{\left|\omega_{D_{2} \tau}\left(s, x_{s}, \xi, x_{s}^{k}, \xi+h_{k}^{\xi}, z_{s}^{k, h}\right)\right|}{\left(\left|x_{s}^{k}-x_{s}\right|_{C}+\left|h_{k}^{\xi}\right|_{\Gamma}\right)\left|z_{s}^{k, h}\right|_{C}} \\
& \rightarrow 0, \quad k \rightarrow \infty .
\end{aligned}
$$

Note that for $s, k$ and $h$ such that $\left|x_{s}^{k}-x_{s}\right|_{C}+\left|h_{k}^{\xi}\right|_{\Gamma}=0$ or $\left|z_{s}^{k, h}\right|_{C}=0, \mid \omega_{D_{2} \tau}\left(s, x_{s}, \xi, x_{s}^{k}, \xi+\right.$ $\left.h_{k}^{\xi}, z_{s}^{k, h}\right) \mid=0$. Therefore the Dominated Convergence Theorem implies (5.26).

The proof of (5.27) is similar.

For a.e. $s \in[0, \alpha], h, y \in \Gamma$ we introduce the bilinear operators by

$$
\begin{aligned}
G(s)\left\langle\left(h^{\varphi}, h^{\xi}\right),\left(y^{\varphi}, y^{\xi}\right)\right\rangle:= & D_{22} \tau\left(s, x_{s}, \xi\right)\left\langle h^{\varphi}, y^{\varphi}\right\rangle+D_{23} \tau\left(s, x_{s}, \xi\right)\left\langle h^{\varphi}, y^{\xi}\right\rangle \\
& +D_{32} \tau\left(s, x_{s}, \xi\right)\left\langle h^{\xi}, y^{\varphi}\right\rangle+D_{33} \tau\left(s, x_{s}, \xi\right)\left\langle h^{\xi}, y^{\xi}\right\rangle, \\
H(s)\left\langle\left(h^{\varphi}, h^{\xi}\right),\left(y^{\varphi}, y^{\xi}\right)\right\rangle:= & -A\left(s, h^{\varphi}, h^{\xi}\right) F\left(s, y^{\varphi}, y^{\xi}\right)-\dot{x}(u(s)) G(s)\left\langle\left(h^{\varphi}, h^{\xi}\right),\left(y^{\varphi}, y^{\xi}\right)\right\rangle \\
& -\dot{h}^{\varphi}\left(-\tau\left(s, x_{s}, \xi\right)\right) A\left(s, y^{\varphi}, y^{\xi}\right),
\end{aligned}
$$

and

$$
\begin{aligned}
B(s)\langle h, y\rangle:= & D_{22} f(\mathbf{v}(s))\left\langle h^{\varphi}, y^{\varphi}\right\rangle+D_{23} f(\mathbf{v}(s))\left\langle h^{\varphi}, E\left(s, y^{\varphi}, y^{\xi}\right)\right\rangle+D_{24} f(\mathbf{v}(s))\left\langle h^{\varphi}, y^{\theta}\right\rangle \\
& +D_{32} f(\mathbf{v}(s))\left\langle E\left(s, h^{\varphi}, h^{\xi}\right), y^{\varphi}\right\rangle+D_{33} f(\mathbf{v}(s))\left\langle E\left(s, h^{\varphi}, h^{\xi}\right), E\left(s, y^{\varphi}, y^{\xi}\right)\right\rangle \\
& +D_{34} f(\mathbf{v}(s))\left\langle E\left(s, h^{\varphi}, h^{\xi}\right), y^{\theta}\right\rangle+D_{42} f(\mathbf{v}(s))\left\langle h^{\theta}, y^{\varphi}\right\rangle \\
& +D_{43} f(\mathbf{v}(s))\left\langle h^{\theta}, E\left(s, y^{\varphi}, y^{\xi}\right)\right\rangle+D_{44} f(\mathbf{v}(s))\left\langle h^{\theta}, y^{\theta}\right\rangle \\
& +D_{3} f(\mathbf{v}(s)) H(s)\left\langle\left(h^{\varphi}, h^{\xi}\right),\left(y^{\varphi}, y^{\xi}\right)\right\rangle .
\end{aligned}
$$

Note that $G, H$ and $B$ correspond to $\gamma$, but this dependence is omitted for simplicity in the notation.

For $\gamma \in P_{2}$ consider the corresponding solution $x$ of the IVP (1.1)-(1.2), and let $z^{h}$ and $z^{y}$ be the solutions of the IVP (4.13)-(4.14) corresponding to a fixed $h, y \in \Gamma$. We consider the IVP

$$
\begin{aligned}
& \dot{w}(t)=L(t, x)\left(w_{t}, 0,0\right)+B(t)\left\langle\left(z_{t}^{h}, h^{\theta}, h^{\xi}\right),\left(z_{t}^{y}, y^{\theta}, y^{\xi}\right)\right\rangle, \quad \text { a.e. } t \in[0, \alpha], \\
& w(t)=0, \quad t \in[-r, 0] .
\end{aligned}
$$

The IVP (5.28)-(5.29) is a Carathéodory type inhomogeneous linear delay system with timedependent but state-independent delays. It is easy to see that under assumptions (A1) (i)-(vi), (A2) (i)-(vii) the IVP (5.28)-(5.29) has a unique solution on $[-r, \alpha]$, which will be denoted by $w^{h, y}(t):=w(t, \gamma, h, y)$. It is easy to see that $\Gamma \times \Gamma \rightarrow \mathbb{R}^{n},(h, y) \mapsto w(t, \gamma, h, y)$ is a bilinear map for a fixed $t \in[0, \alpha]$ and $\gamma \in P_{2}$. In Lemma 5.13 below we will show that this bilinear map is bounded.

We need the further notation

$$
q^{k, h}(s):=z^{k, h}(s)-z^{h}(s)-w^{h, h_{k}}(s), \quad s \in[-r, \alpha] .
$$


Lemma 5.9 Assume (A2) (i)-(vi) and (H). Then there exists $K_{10} \geq 0$ such that

$$
\left|A^{k}\left(s, z_{s}^{j, h}, h^{\xi}\right)-A\left(s, z_{s}^{j, h}, h^{\xi}\right)\right| \leq K_{10}|h|_{\Gamma}\left|h_{k}\right|_{\Gamma}, \quad s \in[0, \alpha], k \in \mathbb{N}, j \in \mathbb{N}_{0},
$$

and there exists a sequence $c_{2, k} \geq 0$ satisfying $c_{2, k} \rightarrow 0$ as $k \rightarrow \infty$ such that

$$
\left|A^{k}\left(s, z_{s}^{k, h}, h^{\xi}\right)-A\left(s, z_{s}^{h}, h^{\xi}\right)\right| \leq c_{2, k}|h|_{\Gamma}, \quad s \in[0, \alpha], k \in \mathbb{N} .
$$

Proof Let $L_{5}=L_{5}\left(\alpha, M_{1}, M_{3}\right)$ be defined by (A2) (vi). To show (5.31) we use (3.2), (4.15), (4.20) and (A2) (vi) to get

$$
\begin{aligned}
& \left|A^{k}\left(s, z_{s}^{j, h}, h^{\xi}\right)-A\left(s, z_{s}^{j, h}, h^{\xi}\right)\right| \\
& \quad \leq\left|D_{2} \tau\left(s, x_{s}^{k}, \xi+h_{k}^{\xi}\right) z_{s}^{j, h}-D_{2} \tau\left(s, x_{s}, \xi\right) z_{s}^{j, h}\right|+\left|D_{3} \tau\left(s, x_{s}^{k}, \xi+h_{k}^{\xi}\right) h^{\xi}-D_{3} \tau\left(s, x_{s}, \xi\right) h^{\xi}\right| \\
& \quad \leq L_{5}(L+1)\left|h_{k}\right|_{\Gamma} N_{1}|h|_{\Gamma}+L_{5}(L+1)\left|h_{k}\right|_{\Gamma}|h|_{\Gamma}, \quad s \in[0, \alpha], k \in \mathbb{N}, j \in \mathbb{N}_{0},
\end{aligned}
$$

which yields (5.30). Using (4.25), (5.31) and (A2) (ii) we get

$$
\begin{aligned}
& \left|A^{k}\left(s, z_{s}^{k, h}, h^{\xi}\right)-A\left(s, z_{s}^{h}, h^{\xi}\right)\right| \\
& \quad \leq\left|A^{k}\left(s, z_{s}^{k, h}, h^{\xi}\right)-A\left(s, z_{s}^{k, h}, h^{\xi}\right)\right|+\left|A\left(s, z_{s}^{k, h}, h^{\xi}\right)-A\left(s, z_{s}^{h}, h^{\xi}\right)\right| \\
& \quad \leq K_{10}|h|_{\Gamma}\left|h_{k}\right|_{\Gamma}+\left|D_{2} \tau\left(s, x_{s}, \xi\right)\left(z_{s}^{k, h}-z_{s}^{h}\right)\right| \\
& \quad \leq K_{10}\left|h_{k}\right|_{\Gamma}|h|_{\Gamma}+L_{2} c_{1, k} N_{1}|h|_{\Gamma}, \quad s \in[0, \alpha], k \in \mathbb{N},
\end{aligned}
$$

therefore (5.31) holds.

Lemma 5.10 Assume (A1) (i)-(v), (A2) (i)-(vii), (H) and $\gamma \in \mathcal{P}$. Then

$$
\begin{gathered}
A^{k}\left(s, z_{s}^{k, h}, h^{\xi}\right)-A\left(s, z_{s}^{h}, h^{\xi}\right)-G(s)\left\langle\left(z_{s}^{h}, h^{\xi}\right),\left(z_{s}^{h_{k}}, h_{k}^{\xi}\right)\right\rangle-A\left(s, w_{s}^{h, h_{k}}, 0\right) \\
=A\left(s, q_{s}^{k, h}, 0\right)+g_{3}^{k, h}(s), \quad s \in[0, \alpha], h \in \Gamma, k \in \mathbb{N},
\end{gathered}
$$

where

$$
\begin{aligned}
g_{3}^{k, h}(s):= & D_{22} \tau\left(s, x_{s}, \xi\right)\left\langle z_{s}^{k, h}-z_{s}^{h}, x_{s}^{k}-x_{s}\right\rangle+D_{22} \tau\left(s, x_{s}, \xi\right)\left\langle z_{s}^{h}, p_{s}^{k}\right\rangle \\
& +D_{23} \tau\left(s, x_{s}, \xi\right)\left\langle z_{s}^{k, h}-z_{s}^{h}, h_{k}^{\xi}\right\rangle+D_{32} \tau\left(s, x_{s}, \xi\right)\left\langle h^{\xi}, p_{s}^{k}\right\rangle \\
& +\omega_{D_{2} \tau}\left(s, x_{s}, \xi, x_{s}^{k}, \xi+h_{k}^{\xi}, z_{s}^{k, h}\right)+\omega_{D_{3} \tau}\left(s, x_{s}, \xi, x_{s}^{k}, \xi+h_{k}^{\xi}, h^{\xi}\right)
\end{aligned}
$$

satisfies

$$
\lim _{k \rightarrow \infty} \sup _{\substack{h \neq 0 \\ h \in \Gamma}} \frac{1}{|h|_{\Gamma}\left|h_{k}\right|_{\Gamma}} \int_{0}^{\alpha}\left|g_{3}^{k, h}(s)\right| d s=0 ;
$$

and if $h_{k} \in \Gamma_{2}$ for $k \in \mathbb{N}$, then

$$
\begin{gathered}
E^{k}\left(s, z_{s}^{k, h}, h^{\xi}\right)-E\left(s, z_{s}^{h}, h^{\xi}\right)-H(s)\left\langle\left(z_{s}^{h}, h^{\xi}\right),\left(z_{s}^{h_{k}}, h_{k}^{\xi}\right)\right\rangle-E\left(s, w_{s}^{h, h_{k}}, 0\right) \\
=E\left(s, q_{s}^{k, h}, 0\right)+g_{4}^{k, h}(s), \quad \text { a.e. } s \in[0, \alpha], h \in \Gamma, k \in \mathbb{N}
\end{gathered}
$$

with

$$
\begin{aligned}
g_{4}^{k, h}(s):= & -\left[\dot{x}^{k}\left(u^{k}(s)\right)-\dot{x}(u(s))\right]\left[A^{k}\left(s, z_{s}^{k, h}, h^{\xi}\right)-A\left(s, z_{s}^{k, h}, h^{\xi}\right)\right]-g_{2}^{k}(s) A\left(s, z_{s}^{k, h}, h^{\xi}\right) \\
& -\dot{x}(u(s)) g_{3}^{k, h}(s)+z^{k, h}\left(u^{k}(s)\right)-z^{h}\left(u^{k}(s)\right)-\left[z^{k, h}(u(s))-z^{h}(u(s))\right] \\
& +z^{h}\left(u^{k}(s)\right)-z^{h}(u(s))-\dot{z}^{h}(u(s))\left(u^{k}(s)-u(s)\right) \\
& +\dot{z}^{h}(u(s))\left(u^{k}(s)-u(s)+A\left(s, z_{s}^{h_{k}}, h_{k}^{\xi}\right)\right)
\end{aligned}
$$


satisfying

$$
\lim _{k \rightarrow \infty} \sup _{\substack{h \neq 0 \\ h \in \Gamma_{2}}} \frac{1}{|h|_{\Gamma_{2}}\left|h_{k}\right| \Gamma_{2}} \int_{0}^{\alpha}\left|g_{4}^{k, h}(s)\right| d s=0 .
$$

Proof The definitions of $A^{k}, A, G, g_{3}^{k, h}, \omega_{D_{2} \tau}, \omega_{D_{3} \tau}$ and relation

$$
A\left(s, z_{s}^{k, h}, h^{\xi}\right)-A\left(s, z_{s}^{h}, h^{\xi}\right)-A\left(s, w_{s}^{h, h_{k}}, 0\right)=A\left(s, z_{s}^{k, h}-z_{s}^{h}-w_{s}^{h, h_{k}}, 0\right)
$$

yield

$$
\begin{aligned}
A^{k}\left(s, z_{s}^{k, h},\right. & \left.h^{\xi}\right)-A\left(s, z_{s}^{h}, h^{\xi}\right)-G(s)\left\langle\left(z_{s}^{h}, h^{\xi}\right),\left(z_{s}^{h_{k}}, h_{k}^{\xi}\right)\right\rangle-A\left(s, w_{s}^{h, h_{k}}, 0\right) \\
= & A^{k}\left(s, z_{s}^{k, h}, h^{\xi}\right)-A\left(s, z_{s}^{k, h}, h^{\xi}\right)-G(s)\left\langle\left(z_{s}^{h}, h^{\xi}\right),\left(z_{s}^{h_{k}}, h_{k}^{\xi}\right)\right\rangle+A\left(s, q_{s}^{k, h}, 0\right) \\
= & D_{2} \tau\left(s, x_{s}^{k}, \xi+h_{k}^{\xi}\right) z_{s}^{k, h}-D_{2} \tau\left(s, x_{s}, \xi\right) z_{s}^{k, h}-D_{22} \tau\left(s, x_{s}, \xi\right)\left\langle z_{s}^{k, h}, x_{s}^{k}-x_{s}\right\rangle \\
& \quad-D_{23} \tau\left(s, x_{s}, \xi\right)\left\langle z_{s}^{k, h}, h_{k}^{\xi}\right\rangle+D_{22} \tau\left(s, x_{s}, \xi\right)\left\langle z_{s}^{k, h}-z_{s}^{h}, x_{s}^{k}-x_{s}\right\rangle \\
& +D_{22} \tau\left(s, x_{s}, \xi\right)\left\langle z_{s}^{h}, p_{s}^{k}\right\rangle+D_{23} \tau\left(s, x_{s}, \xi\right)\left\langle z_{s}^{k, h}-z_{s}^{h}, h_{k}^{\xi}\right\rangle \\
& +D_{3} \tau\left(t, x_{s}^{k}, \xi+h_{k}^{\xi}\right) h^{\xi}-D_{3} \tau\left(s, x_{s}, \xi\right) h^{\xi}-D_{32} \tau\left(s, x_{s}, \xi\right)\left\langle h^{\xi}, x_{s}^{k}-x_{s}\right\rangle \\
& -D_{33} \tau\left(s, x_{s}, \xi\right)\left\langle h^{\xi}, h_{k}^{\xi}\right\rangle+D_{32} \tau\left(s, x_{s}, \xi\right)\left\langle h^{\xi}, p_{s}^{k}\right\rangle+A\left(s, q_{s}^{k, h}, 0\right) \\
= & A\left(s, q_{s}^{k, h}, 0\right)+g_{3}^{k, h}(s) .
\end{aligned}
$$

Let $L_{5}=L_{5}\left(\alpha, M_{1}, M_{3}\right)$ be defined by (A2) (vi). Then we have by (3.2), (4.15) and (4.25)

$$
\begin{aligned}
\int_{0}^{\alpha}\left|g_{3}^{k, h}(s)\right| d s \leq & \alpha L_{5} c_{1, k} N_{1}|h|_{\Gamma} L\left|h_{k}\right|_{\Gamma}+\alpha L_{5} N_{1}|h|_{\Gamma} \max _{s \in[0, \alpha]}\left|p_{s}^{k}\right|_{C}+\alpha L_{5} c_{1, k} N_{1}|h|_{\Gamma}\left|h_{k}\right|_{\Gamma} \\
& +\alpha L_{5}|h|_{\Gamma} \max _{s \in[0, \alpha]}\left|p_{s}^{k}\right|_{C}+\int_{0}^{\alpha}\left|\omega_{D_{2} \tau}\left(s, x_{s}, \xi, x_{s}^{k}, \xi+h_{k}^{\xi}, z_{s}^{k, h}\right)\right| d s \\
& +\int_{0}^{\alpha}\left|\omega_{D_{3} \tau}\left(s, x_{s}, \xi, x_{s}^{k}, \xi+h_{k}^{\xi}, h^{\xi}\right)\right| d s .
\end{aligned}
$$

Hence $c_{1, k} \rightarrow 0$ as $k \rightarrow \infty,(5.18)$, (5.26) and (5.27) imply (5.33).

Relation

$$
E\left(s, z_{s}^{k, h}, h^{\xi}\right)-E\left(s, z_{s}^{h}, h^{\xi}\right)-E\left(s, w_{s}^{h, h_{k}}, 0\right)=E\left(s, z_{s}^{k, h}-z_{s}^{h}-w_{s}^{h, h_{k}}, 0\right)
$$

and the definition of $E, E^{k}$ and $H$ give

$$
\begin{aligned}
E^{k}\left(s, z_{s}^{k, h},\right. & \left.h^{\xi}\right)-E\left(s, z_{s}^{h}, h^{\xi}\right)-H(s)\left\langle\left(z_{s}^{h}, h^{\xi}\right),\left(z_{s}^{h_{k}}, h_{k}^{\xi}\right)\right\rangle-E\left(s, w_{s}^{h, h_{k}}, 0\right) \\
= & E^{k}\left(s, z_{s}^{k, h}, h^{\xi}\right)-E\left(s, z_{s}^{k, h}, h^{\xi}\right)-H(s)\left\langle\left(z_{s}^{h}, h^{\xi}\right),\left(z_{s}^{h_{k}}, h_{k}^{\xi}\right)\right\rangle+E\left(s, q_{s}^{k, h}, 0\right) \\
= & -\dot{x}^{k}\left(u^{k}(s)\right) A^{k}\left(s, z_{s}^{k, h}, h^{\xi}\right)+\dot{x}(u(s)) A\left(s, z_{s}^{k, h}, h^{\xi}\right)+z^{k, h}\left(u^{k}(s)\right)-z^{k, h}(u(s)) \\
& +A\left(s, z_{s}^{h}, h^{\xi}\right) F\left(s, z_{s}^{h_{k}}, h_{k}^{\xi}\right)+\dot{x}(u(s)) G(s)\left\langle\left(z_{s}^{h}, h^{\xi}\right),\left(z_{s}^{h_{k}}, h_{k}^{\xi}\right)\right\rangle \\
& +\dot{z}^{h}(u(s)) A\left(s, z_{s}^{h_{k}}, h_{k}^{\xi}\right)-E\left(s, q_{s}^{k, h}, 0\right) \\
= & -\left[\dot{x}^{k}\left(u^{k}(s)\right)-\dot{x}(u(s))\right]\left[A^{k}\left(s, z_{s}^{k, h}, h^{\xi}\right)-A\left(s, z_{s}^{k, h}, h^{\xi}\right)\right] \\
& -\left[\dot{x}^{k}\left(u^{k}(s)\right)-\dot{x}(u(s))-F\left(s, z_{s}^{h_{k}}, h_{k}^{\xi}\right)\right] A\left(s, z_{s}^{k, h}, h^{\xi}\right) \\
& -\dot{x}(u(s))\left[A^{k}\left(s, z_{s}^{k, h}, h^{\xi}\right)-A\left(s, z_{s}^{k, h}, h^{\xi}\right)-G(s)\left\langle\left(z_{s}^{h}, h^{\xi}\right),\left(z_{s}^{h_{k}}, h_{k}^{\xi}\right)\right\rangle\right] \\
& +z^{k, h}\left(u^{k}(s)\right)-z^{h}\left(u^{k}(s)\right)-\left[z^{k, h}(u(s))-z^{h}(u(s))\right] \\
& +z^{h}\left(u^{k}(s)\right)-z^{h}(u(s))-\dot{z}^{h}(u(s))\left(u^{k}(s)-u(s)\right) \\
& +\dot{z}^{h}(u(s))\left(u^{k}(s)-u(s)+A\left(s, z_{s}^{h_{k}}, h_{k}^{\xi}\right)\right)+E\left(s, q_{s}^{k, h}, 0\right)
\end{aligned}
$$


which, together with (5.23) and (5.32), yields (5.34).

To prove (5.35) first note that by (3.2), (3.4) and (5.2)

$$
\begin{aligned}
\left|\dot{x}^{k}\left(u^{k}(s)\right)-\dot{x}(u(s))\right| & \leq\left|\dot{x}^{k}\left(u^{k}(s)\right)-\dot{x}\left(u^{k}(s)\right)\right|+\left|\dot{x}\left(u^{k}(s)\right)-\dot{x}(u(s))\right| \\
& \leq L\left|h_{k}\right|_{\Gamma}+K_{4} K_{0}\left|h_{k}\right|_{\Gamma} .
\end{aligned}
$$

Hence (5.30) and (5.36) give

$$
\lim _{k \rightarrow \infty} \sup _{\substack{h \neq 0 \\ h \in \Gamma}} \frac{1}{|h|_{\Gamma}\left|h_{k}\right|_{\Gamma}} \int_{0}^{\alpha}\left|\dot{x}^{k}\left(u^{k}(s)\right)-\dot{x}(u(s))\right|\left|A^{k}\left(s, z_{s}^{k, h}, h^{\xi}\right)-A\left(s, z_{s}^{k, h}, h^{\xi}\right)\right| d s=0 .
$$

Relations(3.1), (5.9), (5.24) and (5.33) imply for $h_{k} \in \Gamma_{2}$ for $k \in \mathbb{N}$

$$
\lim _{k \rightarrow \infty} \sup _{\substack{h \neq 0 \\ h \in \Gamma}} \frac{1}{|h|_{\Gamma}\left|h_{k}\right|_{\Gamma_{2}}} \int_{0}^{\alpha}\left|g_{2}^{k}(s) A\left(s, z_{s}^{k, h}, h^{\xi}\right)\right| d s \leq \lim _{k \rightarrow \infty} \frac{K_{6}}{\left|h_{k}\right|_{\Gamma_{2}}} \int_{0}^{\alpha}\left|g_{2}^{k}(s)\right| d s=0
$$

and

$$
\lim _{k \rightarrow \infty} \sup _{\substack{h \neq 0 \\ h \in \Gamma}} \frac{1}{|h|_{\Gamma}\left|h_{k}\right|_{\Gamma}} \int_{0}^{\alpha}\left|\dot{x}(u(s)) g_{3}^{k, h}(s)\right| d s \leq \lim _{k \rightarrow \infty} \frac{N}{|h|_{\Gamma}\left|h_{k}\right|_{\Gamma}} \int_{0}^{\alpha}\left|g_{3}^{k, h}(s)\right| d s=0 .
$$

The above limits and (5.14), (5.16), $\left|\dot{z}^{h}(u(s))\right| \leq N_{2}|h|_{\Gamma_{2}}$ and (5.20) yield (5.35).

Lemma 5.11 Assume (A2) (i)-(vii), (H) and $\gamma \in \mathcal{P}$. Then there exist $K_{11}=K_{11}(\gamma) \geq 0$ and a nonnegative sequence $c_{3, k}=c_{3, k}(\gamma)$ satisfying $c_{3, k} \rightarrow 0$ as $k \rightarrow \infty$ such that

$$
\begin{aligned}
\left|F\left(s, z_{s}^{h}, h^{\xi}\right)\right| & \leq K_{11}|h|_{\Gamma}, \quad \text { a.e. } s \in[0, \alpha], h \in \Gamma, \\
\left|E^{k}\left(s, z_{s}^{k, h}, h^{\xi}\right)-E\left(s, z_{s}^{h}, h^{\xi}\right)\right| & \leq c_{3, k}|h|_{\Gamma}, \quad \text { a.e. } s \in[0, \alpha], k \in \mathbb{N},
\end{aligned}
$$

and, if in addition, (A2) (viii) holds, there exists a nonnegative sequence $c_{4, k}=c_{4, k}(\gamma)$ satisfying $c_{4, k} \rightarrow 0$ as $k \rightarrow \infty$ such that

$$
\int_{0}^{\alpha}\left|F^{k}\left(s, z_{s}^{k, h}, h^{\xi}\right)-F\left(s, z_{s}^{h}, h^{\xi}\right)\right| d s \leq c_{4, k}|h|_{\Gamma_{2}}, \quad \text { a.e. } s \in[0, \alpha], k \in \mathbb{N}, h \in \Gamma_{2} .
$$

Proof The definition of $F,(5.1)$ and (5.9) imply immediately (5.37) with $K_{11}:=K_{4} K_{6}+1$.

Relations (3.1), (3.2), (3.4), (4.15), (4.16), (4.25), (5.9), (5.31), (5.36) and (H2) (ii) yield for a.e. $s \in[0, \alpha]$

$$
\begin{aligned}
\mid E^{k}\left(s, z_{s}^{k, h},\right. & \left.h^{\xi}\right)-E\left(s, z_{s}^{h}, h^{\xi}\right) \mid \\
\leq & \left|\dot{x}^{k}\left(u^{k}(s)\right)-\dot{x}(u(s))\right|\left|A^{k}\left(s, z_{s}^{k, h}, h^{\xi}\right)\right| \\
& +|\dot{x}(u(s))|\left|A^{k}\left(s, z_{s}^{k, h}, h^{\xi}\right)-A\left(s, z_{s}^{h}, h^{\xi}\right)\right|+\left|z^{k, h}\left(u^{k}(s)\right)-z^{h}\left(u^{k}(s)\right)\right| \\
& +\left|z^{h}\left(u^{k}(s)\right)-z^{h}(u(s))\right| \\
\leq & \left(L+K_{4} K_{0}\right)\left|h_{k}\right|_{\Gamma} K_{6}|h|_{\Gamma}+N c_{2, k}|h|_{\Gamma}+c_{1, k} N_{1}|h|_{\Gamma}+N_{2}|h|_{\Gamma} K_{0}\left|h_{k}\right|_{\Gamma},
\end{aligned}
$$

which proves (5.38). 


$$
\begin{aligned}
&\left|F^{k}\left(s, z_{s}^{h}, h^{\xi}\right)-F\left(s, z_{s}^{h}, h^{\xi}\right)\right| \\
& \leq\left(\left|\ddot{x}^{k}\left(u^{k}(s)\right)-\ddot{x}\left(u^{k}(s)\right)\right|+\left|\ddot{x}\left(u^{k}(s)\right)-\ddot{x}(u(s))\right|\right)\left|A^{k}\left(s, z_{s}^{h}, h^{\xi}\right)\right| \\
&+|\ddot{x}(u(s))|\left|A^{k}\left(s, z_{s}^{h}, h^{\xi}\right)-A^{k}\left(s, z_{s}^{h}, h^{\xi}\right)\right|+\left|\dot{z}^{h}\left(u^{k}(s)\right)-\dot{z}^{h}(u(s))\right| .
\end{aligned}
$$

For $t \in(0, \alpha]$ we have by (A2) (viii) that

$$
\begin{aligned}
\left|\ddot{x}^{k}(t)-\ddot{x}(t)\right| & =\left|\frac{d}{d t} f\left(t, x_{t}^{k}, x^{k}\left(u^{k}(t)\right), \theta+h_{k}^{\theta}\right)-\frac{d}{d t} f\left(t, x_{t}, x(u(t)), \theta\right)\right| \\
& \leq L_{6}\left(\left|x_{t}^{k}-x_{t}\right|_{C}+\left|h_{k}^{\theta}\right|_{\Theta}+\left|h_{k}^{\xi}\right|_{\Xi}\right) \\
& \leq L_{6}(L+1)\left|h_{k}\right|_{\Gamma} .
\end{aligned}
$$

For $t \in[-r, 0)$ and $h \in \Gamma_{2}$ we get

$$
\left|\ddot{x}^{k}(t)-\ddot{x}(t)\right|=\left|\ddot{h}_{k}^{\varphi}(t)\right| \leq\left|h_{k}\right|_{\Gamma_{2}} .
$$

Using that $\ddot{x} \in L^{\infty}\left([-r, \alpha], \mathbb{R}^{n}\right)$, similarly to (4.24) we can argue that

$$
\lim _{k \rightarrow \infty} \int_{0}^{\alpha}\left|\ddot{x}\left(u^{k}(s)\right)-\ddot{x}(u(s))\right| d s=0 .
$$

Then the above relations, $|\ddot{x}(u(s))| \leq K_{4}$ for a.e. $s \in[0, \alpha],(5.9),(5.13)$ and (5.30) yield (5.39) $\square$

For a.e. $s \in[0, \alpha], h, y \in \Gamma$ and $k \in \mathbb{N}$ we introduce the bilinear operators by

$$
\begin{aligned}
G^{k}(s)\left\langle\left(h^{\varphi}, h^{\xi}\right),\left(y^{\varphi}, y^{\xi}\right)\right\rangle:= & D_{22} \tau\left(s, x_{s}^{k}, \xi+h_{k}^{\xi}\right)\left\langle h^{\varphi}, y^{\varphi}\right\rangle+D_{23} \tau\left(s, x_{s}^{k}, \xi+h_{k}^{\xi}\right)\left\langle h^{\varphi}, y^{\xi}\right\rangle \\
& +D_{32} \tau\left(s, x_{s}^{k}, \xi+h_{k}^{\xi}\right)\left\langle h^{\xi}, y^{\varphi}\right\rangle+D_{33} \tau\left(s, x_{s}^{k}, \xi+h_{k}^{\xi}\right)\left\langle h^{\xi}, y^{\xi}\right\rangle, \\
H^{k}(s)\left\langle\left(h^{\varphi}, h^{\xi}\right),\left(y^{\varphi}, y^{\xi}\right)\right\rangle:= & -A^{k}\left(s, h^{\varphi}, h^{\xi}\right) F^{k}\left(s, y^{\varphi}, y^{\xi}\right) \\
& -\dot{x}^{k}\left(u^{k}(s)\right) G^{k}(s)\left\langle\left(h^{\varphi}, h^{\xi}\right),\left(y^{\varphi}, y^{\xi}\right)\right\rangle \\
& -\dot{h}^{\varphi}\left(-\tau\left(s, x_{s}^{k}, \xi+h_{k}^{\xi}\right)\right) A^{k}\left(s, y^{\varphi}, y^{\xi}\right),
\end{aligned}
$$

and

$$
\begin{aligned}
B^{k}(s)\langle h, y\rangle:= & D_{22} f\left(\mathbf{v}^{k}(s)\right)\left\langle h^{\varphi}, y^{\varphi}\right\rangle+D_{23} f\left(\mathbf{v}^{k}(s)\right)\left\langle h^{\varphi}, E^{k}\left(s, y^{\varphi}, y^{\xi}\right)\right\rangle \\
& +D_{24} f\left(\mathbf{v}^{k}(s)\right)\left\langle h^{\varphi}, y^{\theta}\right\rangle+D_{32} f\left(\mathbf{v}^{k}(s)\right)\left\langle E^{k}\left(s, h^{\varphi}, h^{\xi}\right), y^{\varphi}\right\rangle \\
& +D_{33} f\left(\mathbf{v}^{k}(s)\right)\left\langle E^{k}\left(s, h^{\varphi}, h^{\xi}\right), E^{k}\left(s, y^{\varphi}, y^{\xi}\right)\right\rangle \\
& +D_{34} f\left(\mathbf{v}^{k}(s)\right)\left\langle E^{k}\left(s, h^{\varphi}, h^{\xi}\right), y^{\theta}\right\rangle+D_{42} f\left(\mathbf{v}^{k}(s)\right)\left\langle h^{\theta}, y^{\varphi}\right\rangle \\
& +D_{43} f\left(\mathbf{v}^{k}(s)\right)\left\langle h^{\theta}, E^{k}\left(s, y^{\varphi}, y^{\xi}\right)\right\rangle+D_{44} f\left(\mathbf{v}^{k}(s)\right)\left\langle h^{\theta}, y^{\theta}\right\rangle \\
& +D_{3} f\left(\mathbf{v}^{k}(s)\right) H^{k}(s)\left\langle\left(h^{\varphi}, h^{\xi}\right),\left(y^{\varphi}, y^{\xi}\right)\right\rangle .
\end{aligned}
$$

Lemma 5.12 Assume (A1) (i)-(vi), (A2) (i)-(vii). Then for every $\gamma \in P_{2}$ there exists $K_{12}=$ $K_{12}(\gamma) \geq 0$ such that

$$
\left|B(s)\left\langle\left(z_{s}^{h}, h^{\theta}, h^{\xi}\right),\left(z_{s}^{y}, y^{\theta}, y^{\xi}\right)\right\rangle\right| \leq K_{12}|h|_{\Gamma}|y|_{\Gamma}, \quad \text { a.e. } s \in[-r, \alpha], h, y \in \Gamma, \gamma \in P_{2} .
$$


If in addition (A2) (viii) holds, then for every $\gamma \in P_{2} \cap \mathcal{P}$ there exists a nonnegative sequence $c_{5, k}=c_{5, k}(\gamma)$ such that $c_{5, k} \rightarrow 0$ as $k \rightarrow \infty$, and

$$
\int_{0}^{\alpha}\left|B^{k}(s)\left\langle\left(z_{s}^{h}, h^{\theta}, h^{\xi}\right),\left(z_{s}^{y}, y^{\theta}, y^{\xi}\right)\right\rangle-B(s)\left\langle\left(z_{s}^{h}, h^{\theta}, h^{\xi}\right),\left(z_{s}^{y}, y^{\theta}, y^{\xi}\right)\right\rangle\right| d s \leq c_{5, k}|h|_{\Gamma_{2}}|y|_{\Gamma_{2}},
$$

for $h, y \in \Gamma_{2}$.

Proof Let $L_{3}=L_{3}\left(\alpha, M_{1}, M_{2}, M_{3}\right)$ and $L_{5}=L_{5}\left(\alpha, M_{1}, M_{4}\right)$ be the Lipschitz constants from (A1) (v) and (A2) (vi), respectively. Then the definition of $G,(\mathrm{~A} 2)$ (vi) and (4.15) yield

$$
\left|G(s)\left\langle\left(z_{s}^{h}, h^{\xi}\right),\left(z_{s}^{y}, y^{\xi}\right)\right\rangle\right| \leq 4 L_{5} N_{1}^{2}|h|_{\Gamma}|y|_{\Gamma}, \quad h, y \in \Gamma, \quad s \in[0, \alpha] .
$$

Then definition of $H,(3.1),(4.15),(5.1),(5.9),(5.37)$ and (5.42) imply

$$
\left|H(s)\left\langle\left(z_{s}^{h}, h^{\xi}\right),\left(z_{s}^{y}, y^{\xi}\right)\right\rangle\right| \leq K_{13}|h|_{\Gamma}|y|_{\Gamma}, \quad h, y \in \Gamma, \quad \text { a.e. } s \in[0, \alpha]
$$

with $K_{13}=K_{13}(\gamma):=K_{6}\left(K_{4} K_{6}+1\right)+N 4 L_{5} N_{1}^{2}+K_{6}$. Therefore we have by the definition of $B,(5.11)$ and $(5.43)$

$$
|B(s)\langle h, y\rangle| \leq L_{3}\left(4+4 K_{8}+K_{8}^{2}+K_{13}\right)|h|_{\Gamma}|y|_{\Gamma}, \quad \text { a.e. } s \in[0, \alpha],
$$

which, together with (4.22), yields (5.40).

Define the set $M_{4}^{*}:=\{\xi\} \cup\left\{h_{k}^{\xi}: k \in \mathbb{N}\right\}$. It is easy to show that $M_{4}^{*} \subset M_{4}$ is a compact subset of $\Xi$. Define

$$
\begin{aligned}
\Omega_{2, \tau}(\varepsilon):=\max _{i, j=2,3} \sup \left\{\left|D_{i j} \tau(s, \psi, \eta)-D_{i j} \tau(s, \bar{\psi}, \bar{\eta})\right|_{\mathcal{L}^{2}\left(X_{i} \times X_{j}, \mathbb{R}\right)}:\right. & \\
& \left.s \in[0, \alpha], \psi, \bar{\psi} \in M_{1}, \eta, \bar{\eta} \in M_{4}^{*},|\psi-\bar{\psi}|_{C}+|\eta-\bar{\eta}|_{\Xi} \leq \varepsilon\right\},
\end{aligned}
$$

where $X_{2}:=C$ and $X_{3}:=\Xi$. Assumption (A2) (vii) and the compactness of $[0, \alpha] \times M_{1} \times M_{4}^{*}$ yields that $\Omega_{2, \tau}(\varepsilon) \rightarrow 0$ as $\varepsilon \rightarrow 0+$. Then (4.15) and (4.20) give

$$
\begin{aligned}
\left|\left[G^{k}(s)-G(s)\right]\left\langle\left(z_{s}^{h}, h^{\xi}\right),\left(z_{s}^{y}, y^{\xi}\right)\right\rangle\right| \leq & \left|\left[D_{22} \tau\left(s, x_{s}^{k}, \xi+h_{k}^{\xi}\right)-D_{22} \tau\left(s, x_{s}, \xi\right)\right]\left\langle z_{s}^{h}, z_{s}^{y}\right\rangle\right| \\
& +\left|\left[D_{23} \tau\left(s, x_{s}^{k}, \xi+h_{k}^{\xi}\right)-D_{23} \tau\left(s, x_{s}^{k}, \xi+h_{k}^{\xi}\right)\right]\left\langle z_{s}^{h}, y^{\xi}\right\rangle\right| \\
& +\left|\left[D_{32} \tau\left(s, x_{s}^{k}, \xi+h_{k}^{\xi}\right)-D_{32} \tau\left(s, x_{s}^{k}, \xi+h_{k}^{\xi}\right)\right]\left\langle h^{\xi}, z_{s}^{y}\right\rangle\right| \\
& +\left|\left[D_{33} \tau\left(s, x_{s}^{k}, \xi+h_{k}^{\xi}\right)-D_{33} \tau\left(s, x_{s}^{k}, \xi+h_{k}^{\xi}\right)\right]\left\langle h^{\xi}, y^{\xi}\right\rangle\right| \\
\leq & \Omega_{2, \tau}\left((L+1)\left|h_{k}\right|_{\Gamma}\right)\left(N_{1}+1\right)^{2}|h|_{\Gamma}|y|_{\Gamma}, \quad s \in[0, \alpha] .
\end{aligned}
$$

Relations (3.1), (3.2), (3.4), (4.15), (4.16), (5.9), (5.30), (5.36), (5.37), (5.39), (5.42) and 
(5.44) imply

$$
\begin{aligned}
& \int_{0}^{\alpha}\left|\left[H^{k}(s)-H(s)\right]\left\langle\left(z_{s}^{h}, h^{\xi}\right),\left(z_{s}^{y}, y^{\xi}\right)\right\rangle\right| d s \\
& \leq \int_{0}^{\alpha}\left(\left|\left[A^{k}\left(s, z_{s}^{h}, h^{\xi}\right)-A\left(s, z_{s}^{h}, h^{\xi}\right)\right] F\left(s, z_{s}^{y}, y^{\xi}\right)\right|\right. \\
& \quad+\left|A^{k}\left(s, z_{s}^{h}, h^{\xi}\right)\left[F^{k}\left(s, z_{s}^{y}, y^{\xi}\right)-F\left(s, z_{s}^{y}, y^{\xi}\right)\right]\right| \\
& \quad+\left|\left[\dot{x}^{k}\left(u^{k}(s)\right)-\dot{x}(u(s))\right] G^{k}(s)\left\langle\left(z_{s}^{h}, h^{\xi}\right),\left(z_{s}^{y}, y^{\xi}\right)\right\rangle\right| \\
& \quad+\left|\dot{x}(u(s))\left[G^{k}(s)-G(s)\right]\left\langle\left(z_{s}^{h}, h^{\xi}\right),\left(z_{s}^{y}, y^{\xi}\right)\right\rangle\right| \\
& \quad+\left|\left[\dot{z}^{h}\left(u^{k}(s)\right)-\dot{z}^{h}(u(s))\right] A^{k}\left(s, z_{s}^{y}, y^{\xi}\right)\right| \\
& \left.\quad+\left|\dot{z}^{h}(u(s))\left[A^{k}\left(s, z_{s}^{y}, y^{\xi}\right)-A\left(s, z_{s}^{y}, y^{\xi}\right)\right]\right|\right) d s \\
& \quad \alpha K_{10}|h|_{\Gamma}\left|h_{k}\right|_{\Gamma} K_{11}|y|_{\Gamma}+K_{6}|h|_{\Gamma} c_{4, k}|y|_{\Gamma_{2}}+\left(L+K_{4} K_{0}\right)\left|h_{k}\right|_{\Gamma} 4 L_{5} N_{1}^{2}|h|_{\Gamma}|y|_{\Gamma} \\
& \quad+N \Omega_{2, \tau}\left((L+1)\left|h_{k}\right|_{\Gamma}\right)\left(N_{1}+1\right)^{2}|h|_{\Gamma}|y|_{\Gamma} \\
& \quad+\int_{0}^{\alpha}\left|\dot{z}^{h}\left(u^{k}(s)\right)-\dot{z}^{h}(u(s))\right| d s K_{6}|y|_{\Gamma}+\alpha N_{2}|h|_{\Gamma_{2}} K_{10}|h|_{\Gamma}|y|_{\Gamma} \\
& \leq \quad c_{6, k}|h|_{\Gamma_{2}}|y|_{\Gamma_{2}}
\end{aligned}
$$

with some appropriate sequence $c_{6, k}=c_{6, k}(\gamma)$ satisfying $c_{6, k} \rightarrow 0$ as $k \rightarrow \infty$, where in the last estimate we used (5.13).

Simple manipulations give

$$
\begin{aligned}
\mid\left[B^{k}(s)-B\right. & (s)]\left\langle\left(z_{s}^{h}, h^{\theta}, h^{\xi}\right),\left(z_{s}^{y}, y^{\theta}, y^{\xi}\right)\right\rangle \mid \\
\leq & \left|\left[D_{22} f\left(\mathbf{v}^{k}(s)\right)-D_{22} f(\mathbf{v}(s))\right]\left\langle z_{s}^{h}, z_{s}^{y}\right\rangle\right| \\
& +\left|\left[D_{23} f\left(\mathbf{v}^{k}(s)\right)-D_{23} f(\mathbf{v}(s))\right]\left\langle z_{s}^{h}, E^{k}\left(s, z_{s}^{y}, y^{\xi}\right)\right\rangle\right| \\
& +\left|D_{23} f(\mathbf{v}(s))\left\langle z_{s}^{h}, E^{k}\left(s, z_{s}^{y}, y^{\xi}\right)-E\left(s, z_{s}^{y}, y^{\xi}\right)\right\rangle\right| \\
& +\left|\left[D_{24} f\left(\mathbf{v}^{k}(s)\right)-D_{24} f(\mathbf{v}(s))\right]\left\langle z_{s}^{h}, y^{\theta}\right\rangle\right| \\
& +\left|\left[D_{32} f\left(\mathbf{v}^{k}(s)\right)-D_{32} f(\mathbf{v}(s))\right]\left\langle E^{k}\left(s, z_{s}^{h}, h^{\xi}\right), z_{s}^{y}\right\rangle\right| \\
& +\left|D_{32} f(\mathbf{v}(s))\left\langle E^{k}\left(s, z_{s}^{h}, h^{\xi}\right)-E\left(s, z_{s}^{h}, h^{\xi}\right), z_{s}^{y}\right\rangle\right| \\
& +\left|\left[D_{33} f\left(\mathbf{v}^{k}(s)\right)-D_{33} f(\mathbf{v}(s))\right]\left\langle E^{k}\left(s, z_{s}^{h}, h^{\xi}\right), E^{k}\left(s, z_{s}^{y}, y^{\xi}\right)\right\rangle\right| \\
& +\left|D_{33} f(\mathbf{v}(s))\left\langle E^{k}\left(s, z_{s}^{h}, h^{\xi}\right)-E\left(s, z_{s}^{h}, h^{\xi}\right), E^{k}\left(s, z_{s}^{y}, y^{\xi}\right)\right\rangle\right| \\
& +\left|D_{33} f(\mathbf{v}(s))\left\langle E\left(s, z_{s}^{h}, h^{\xi}\right), E^{k}\left(s, z_{s}^{y}, y^{\xi}\right)-E\left(s, z_{s}^{y}, y^{\xi}\right)\right\rangle\right| \\
& +\left|\left[D_{34} f\left(\mathbf{v}^{k}(s)\right)-D_{34} f(\mathbf{v}(s))\right]\left\langle E^{k}\left(s, z_{s}^{h}, h^{\xi}\right), y^{\theta}\right\rangle\right| \\
& +\left|D_{34} f(\mathbf{v}(s))\left\langle E^{k}\left(s, z_{s}^{h}, h^{\xi}\right)-E\left(s, z_{s}^{h}, h^{\xi}\right), y^{\theta}\right\rangle\right| \\
& +\left|\left[D_{42} f\left(\mathbf{v}^{k}(s)\right)-D_{42} f(\mathbf{v}(s))\right]\left\langle h^{\theta}, z_{s}^{y}\right\rangle\right| \\
& +\mid\left[D_{43} f\left(\mathbf{v}^{k}(s)\right)-D_{43} f(\mathbf{v}(s))\right]\left\langle h^{\theta}, E^{k}\left(s, z_{s}^{y}, y^{\xi}\right)\right\rangle \\
& +\mid D_{43} f(\mathbf{v}(s))\left\langle h^{\theta}, E^{k}\left(s, z_{s}^{y}, y^{\xi}\right)-E\left(s, z_{s}^{y}, y^{\xi}\right)\right\rangle \\
& +\left|\left[D_{44} f\left(\mathbf{v}^{k}(s)\right)-D_{44} f(\mathbf{v}(s))\right]\left\langle h^{\theta}, y^{\theta}\right\rangle\right| \\
& +\left|\left[D_{3} f\left(\mathbf{v}^{k}(s)\right)-D_{3} f(\mathbf{v}(s))\right] H^{k}(s)\left\langle\left(z_{s}^{h}, h^{\xi}\right),\left(z_{s}^{y}, y^{\xi}\right)\right\rangle\right| \\
& +\left|D_{3} f(\mathbf{v}(s))\left[H^{k}(s)-H(s)\right]\left\langle\left(z_{s}^{h}, h^{\xi}\right),\left(z_{s}^{y}, y^{\xi}\right)\right\rangle\right| . \\
& +
\end{aligned}
$$

Define the set $M_{3}^{*}:=\{\theta\} \cup\left\{h_{k}^{\theta}: k \in \mathbb{N}\right\}$. Clearly, $M_{3}^{*} \subset M_{3}$ is a compact subset of $\Theta$. 
Define

$$
\begin{gathered}
\Omega_{2, f}(\varepsilon):=\max _{i, j=2,3,4} \sup \left\{\left|D_{i j} f(s, \psi, v, \eta)-D_{i j} f(s, \bar{\psi}, \bar{v}, \bar{\eta})\right|_{\mathcal{L}^{2}\left(Y_{i} \times Y_{j}, \mathbb{R}\right)}:\right. \\
s \in[0, \alpha], \psi, \bar{\psi} \in M_{1}, v, \bar{v} \in M_{2}, \eta, \bar{\eta} \in M_{3}^{*}, \\
\left.|\psi-\bar{\psi}|_{C}+|v-\bar{v}|+|\eta-\bar{\eta}|_{\Theta} \leq \varepsilon\right\}
\end{gathered}
$$

where $Y_{2}:=C, Y_{3}:=\mathbb{R}^{n}$ and $Y_{4}:=\Theta$. Assumption (A1) (vi) and the compactness of $[0, \alpha] \times$ $M_{1} \times M_{2} \times M_{3}^{*}$ yields that $\Omega_{2, f}(\varepsilon) \rightarrow 0$ as $\varepsilon \rightarrow 0+$. Then combining (5.46) with (4.19), $\left|D_{i j} f\left(\mathbf{v}^{k}(s)\right)-D_{i j} f(\mathbf{v}(s))\right|_{\mathcal{L}^{2}\left(Y_{i} \times Y_{j}, \mathbb{R}^{n}\right)} \leq \Omega_{2, f}\left(K_{3}\left|h_{k}\right|_{\Gamma}\right)$ for $i, j=2,3,4,\left|D_{i} f\left(\mathbf{v}^{k}(s)\right)\right|_{\mathcal{L}\left(Y_{i}, \mathbb{R}^{n}\right)} \leq$ $L_{1}$ for $i=2,3,4, s \in[0, \alpha]$ and $k \in \mathbb{N}_{0}$, (4.15), (5.11), (5.38), (5.43), (5.45) and (5.46). yields $(5.41)$

Lemma 5.13 Assume (A1) (i)-(vi), (A2) (i)-(vii), $\gamma \in P_{2}$. Then there exists $N_{5}=N_{5}(\gamma) \geq 0$ such that the solution of the IVP (5.28)-(5.29) satisfies

$$
\left|w^{h, y}(t)\right| \leq N_{5}|h|_{\Gamma}|y|_{\Gamma}, \quad t \in[-r, \alpha], \quad h, y \in \Gamma .
$$

Proof It follows from (5.28) and (5.29) that

$$
w^{h, y}(t)=\int_{0}^{t} B(s)\left\langle\left(z_{s}^{h}, h^{\theta}, h^{\xi}\right),\left(z_{s}^{y}, y^{\theta}, y^{\xi}\right)\right\rangle d s+\int_{0}^{t} L(s, x)\left(w_{s}^{h, y}, 0,0\right) d s, \quad t \in[0, \alpha] .
$$

Therefore (4.11) and (5.40) yield

$$
\left|w^{h, y}(t)\right| \leq K_{12}|h|_{\Gamma}|y|_{\Gamma}+L_{1} N_{0} \int_{0}^{t}\left|w_{s}^{h, y}\right|_{C} d s, \quad t \in[0, \alpha]
$$

Since $w^{h, y}(t)=0$ for $t \in[-r, 0]$, Lemma 2.1 gives (5.47) with $N_{5}:=K_{12} e^{L_{1} N_{0} \alpha}$.

Lemma 5.14 Assume (A1) (i)-(vi), (A2) (i)-(viii), (H). For $h, y \in \Gamma_{2}$ and $k \in \mathbb{N}$ let $w^{h, y}(t):=$ $w(t, \gamma, h, y)$ and $w^{k, h, y}(t):=w\left(t, \gamma+h_{k}, h, y\right)$ be the solutions of the IVP (5.28)-(5.29). Then there exists a nonnegative sequence $c_{7, k}=c_{7, k}(\gamma)$ such that

$$
\left|w_{t}^{k, h, y}-w_{t}^{h, y}\right|_{C} \leq c_{7, k}|h|_{\Gamma_{2}}|y|_{\Gamma_{2}}, \quad t \in[0, \alpha], \quad h, y \in \Gamma_{2} .
$$

Proof It follows from (4.11), (4.17), (4.26), (5.28), (5.40), (5.36) and (5.47)

$$
\begin{aligned}
& \left|w^{k, h, y}(t)-w^{h, y}(t)\right| \\
& \leq \int_{0}^{t}\left(\left|\left[L\left(s, x^{k}\right)-L(s, x)\right]\left(w_{s}^{k, h, y}, 0,0\right)\right|+\left|L(s, x)\left(w_{s}^{k, h, y}-w_{s}^{h, y}, 0,0\right)\right|\right) d s \\
& \quad+\int_{0}^{t}\left(\left|B^{k}(s)\left\langle\left(z_{s}^{k, h}, h^{\theta}, h^{\xi}\right),\left(z_{s}^{k, y}-z_{s}^{y}, 0,0\right)\right\rangle\right|+\left|B^{k}(s)\left\langle\left(z_{s}^{k, h}-z_{s}^{h}, 0,0\right),\left(z_{s}^{y}, y^{\theta}, y^{\xi}\right)\right\rangle\right|\right. \\
& \left.\quad+\left|B^{k}(s)\left\langle\left(z_{s}^{h}, h^{\theta}, h^{\xi}\right),\left(z_{s}^{y}, y^{\theta}, y^{\xi}\right)\right\rangle-B(s)\left\langle\left(z_{s}^{h}, h^{\theta}, h^{\xi}\right),\left(z_{s}^{y}, y^{\theta}, y^{\xi}\right)\right\rangle\right|\right) d s
\end{aligned}
$$




$$
\begin{aligned}
\leq & \alpha c_{0, k} N_{5}|h|_{\Gamma}|y|_{\Gamma}+L_{1} L_{2} \int_{0}^{\alpha}\left|\dot{x}\left(u^{k}(s)\right)-\dot{x}(u(s))\right| d s N_{5}|h|_{\Gamma}|y|_{\Gamma} \\
& +L_{1} N_{0} \int_{0}^{t}\left|w_{s}^{k, h, y}-w_{s}^{h, y}\right|_{C} d s+2 \alpha K_{12} c_{1, k} N_{1}^{2}|h|_{\Gamma}|y|_{\Gamma}+\alpha c_{5, k}|h|_{\Gamma}|y|_{\Gamma} \\
\leq & c_{8, k}|h|_{\Gamma}|y|_{\Gamma}+L_{1} N_{0} \int_{0}^{t}\left|w_{s}^{k, h, y}-w_{s}^{h, y}\right|_{C} d s,
\end{aligned}
$$

where $c_{8, k}=c_{8, k}(\gamma):=\alpha c_{0, k} N_{5}+L_{1} L_{2}\left(L+K_{4} K_{0}\right) N_{5}\left|h_{k}\right|_{\Gamma}+2 \alpha K_{12} c_{1, k} N_{1}^{2}+\alpha c_{5, k}$. Then Lemma 2.1 is applicable, since $\left|w_{0}^{k, h, y}-w_{0}^{h, y}\right|_{C}=0$, and it yields (5.48) with $c_{7, k}:=c_{8, k} e^{L_{1} N_{0} \alpha} \square$

We define

$$
\begin{aligned}
\omega_{D_{2} f}\left(\mathbf{v}(s), \mathbf{v}^{k}(s), \psi\right):= & D_{2} f\left(\mathbf{v}^{k}(s)\right) \psi-D_{2} f(\mathbf{v}(s)) \psi-D_{22} f(\mathbf{v}(s))\left\langle\psi, x_{s}^{k}-x_{s}\right\rangle \\
& -D_{23} f(\mathbf{v}(s))\left\langle\psi, x^{k}\left(u^{k}(s)\right)-x(u(s))\right\rangle-D_{24} f(\mathbf{v}(s))\left\langle\psi, h_{k}^{\theta}\right\rangle, \\
\omega_{D_{3} f}\left(\mathbf{v}(s), \mathbf{v}^{k}(s), v\right):= & D_{3} f\left(\mathbf{v}^{k}(s)\right) v-D_{3} f(\mathbf{v}(s)) v-D_{32} f(\mathbf{v}(s))\left\langle v, x_{s}^{k}-x_{s}\right\rangle \\
& -D_{33} f(\mathbf{v}(t))\left\langle v, x^{k}\left(u^{k}(s)\right)-x(u(s))\right\rangle-D_{34} f(\mathbf{v}(s))\left\langle v, h_{k}^{\theta}\right\rangle, \\
\omega_{D_{4} f}\left(\mathbf{v}(s), \mathbf{v}^{k}(s), \eta\right):= & D_{4} f\left(\mathbf{v}^{k}(s)\right) \eta-D_{4} f(\mathbf{v}(s)) \eta-D_{42} f(\mathbf{v}(s))\left\langle\eta, x_{s}^{k}-x_{s}\right\rangle \\
& -D_{43} f(\mathbf{v}(s))\left\langle\eta, x^{k}\left(u^{k}(s)\right)-x(u(s))\right\rangle-D_{44} f(\mathbf{v}(s))\left\langle\eta, h_{k}^{\theta}\right\rangle
\end{aligned}
$$

for $s \in[0, \alpha], \psi \in C, v \in \mathbb{R}^{n}$ and $\eta \in \Theta$.

The proof of the following lemma is similar to that of Lemma 5.8.

Lemma 5.15 Assume (A1) (i)-(vi) and (H). Then

$$
\begin{aligned}
& \lim _{k \rightarrow \infty} \sup _{\substack{h \neq 0 \\
h \in \Gamma}} \frac{1}{|h|_{\Gamma}\left|h_{k}\right|_{\Gamma}} \int_{0}^{\alpha}\left|\omega_{D_{2} f}\left(s, x_{s}, x(u(s)), \theta, x_{s}^{k}, x^{k}\left(u^{k}(s)\right), \theta+h_{k}^{\theta}, z_{s}^{k, h}\right)\right| d s=0, \\
& \lim _{k \rightarrow \infty} \sup _{\substack{h \neq 0 \\
h \in \Gamma}} \frac{1}{|h|_{\Gamma}\left|h_{k}\right|_{\Gamma}} \int_{0}^{\alpha}\left|\omega_{D_{3} f}\left(s, x_{s}, x(u(s)), \theta, x_{s}^{k}, x^{k}\left(u^{k}(s)\right), \theta+h_{k}^{\theta}, E^{k}\left(s, z_{s}^{k, h}, h^{\xi}\right)\right)\right| d s=0,
\end{aligned}
$$

and

$$
\lim _{k \rightarrow \infty} \sup _{\substack{h \neq 0 \\ h \in \Gamma}} \frac{1}{|h|_{\Gamma}\left|h_{k}\right|_{\Gamma}} \int_{0}^{\alpha}\left|\omega_{D_{4} f}\left(s, x_{s}, x(u(s)), \theta, x_{s}^{k}, x^{k}\left(u^{k}(s)\right), \theta+h_{k}^{\theta}, h_{k}^{\theta}\right)\right| d s=0 .
$$

Lemma 5.16 Assume (A1) (i)-(vi), (A2) (i)-(vii), (H), $\gamma \in \mathcal{P}$ and $h_{k} \in \Gamma_{2}$ for $k \in \mathbb{N}$. Then

$$
\begin{gathered}
L\left(s, x^{k}\right)\left(z_{s}^{k, h}, h^{\theta}, h^{\xi}\right)-L(s, x)\left(z_{s}^{h}+w_{s}^{h, h_{k}}, h^{\theta}, h^{\xi}\right)-B(s)\left\langle\left(z_{s}^{h}, h^{\theta}, h^{\xi}\right),\left(z_{s}^{h_{k}}, h_{k}^{\theta}, h_{k}^{\xi}\right)\right\rangle \\
=L(s, x)\left(q_{s}^{k, h}, 0,0\right)+g_{5}^{k, h}(s), \quad \text { a.e. } s \in[0, \alpha],
\end{gathered}
$$


where

$$
\begin{aligned}
g_{5}^{k, h}(s):= & D_{22} f(\mathbf{v}(s))\left\langle z_{s}^{k, h}-z_{s}^{h}, x_{s}^{k}-x_{s}\right\rangle+D_{22} f(\mathbf{v}(s))\left\langle z_{s}^{h}, p_{s}^{k}\right\rangle \\
& +D_{23} f(\mathbf{v}(s))\left\langle z_{s}^{k, h}-z_{s}^{h}, x^{k}\left(u^{k}(s)\right)-x(u(s))\right\rangle-D_{23} f(\mathbf{v}(s))\left\langle z_{s}^{h}, g_{1}^{k}(s)\right\rangle \\
& +D_{24} f(\mathbf{v}(s))\left\langle z_{s}^{k, h}-z_{s}^{h}, h_{k}^{\theta}\right\rangle+D_{32} f(\mathbf{v}(s))\left\langle E\left(s, z_{s}^{h}, h^{\xi}\right), p_{s}^{k}\right\rangle \\
& +D_{32} f(\mathbf{v}(s))\left\langle E^{k}\left(s, z_{s}^{k, h}, h^{\xi}\right)-E\left(s, z_{s}^{h}, h^{\xi}\right), x_{s}^{k}-x_{s}\right\rangle \\
& +D_{33} f(\mathbf{v}(s))\left\langle E^{k}\left(s, z_{s}^{k, h}, h^{\xi}\right)-E\left(s, z_{s}^{h}, h^{\xi}\right), x^{k}\left(u^{k}(s)\right)-x(u(s))\right\rangle \\
& +D_{33} f(\mathbf{v}(s))\left\langle E\left(s, z_{s}^{h}, h^{\xi}\right), g_{1}^{k}(s)\right\rangle+D_{3} f(\mathbf{v}(s)) g_{4}^{k, h}(s) \\
& +D_{34} f(\mathbf{v}(s))\left\langle E^{k}\left(s, z_{s}^{k, h}, h^{\xi}\right)-E\left(s, z_{s}^{h}, h^{\xi}\right), h_{k}^{\theta}\right\rangle \\
& +D_{42} f(\mathbf{v}(s))\left\langle h^{\theta}, p_{s}^{k}\right\rangle+D_{43} f(\mathbf{v}(s))\left\langle h^{\theta}, g_{1}^{k}(s)\right\rangle+\omega_{D_{2} f}\left(\mathbf{v}(s), \mathbf{v}^{k}(s), z_{s}^{k, h}\right) \\
& +\omega_{D_{3} f}\left(\mathbf{v}(s), \mathbf{v}^{k}(s), E^{k}\left(s, z_{s}^{k, h}, h^{\xi}\right)\right)+\omega_{D_{4} f}\left(\mathbf{v}(s), \mathbf{v}^{k}(s), h_{k}^{\theta}\right)
\end{aligned}
$$

satisfies

$$
\lim _{k \rightarrow \infty} \sup _{\substack{h \neq 0 \\ h \in \Gamma_{2}}} \frac{1}{|h|_{\Gamma_{2}}\left|h_{k}\right|_{\Gamma_{2}}} \int_{0}^{\alpha}\left|g_{5}^{k, h}(s)\right| d s=0 .
$$

Proof Straightforward manipulations yield for a.e. $s \in[0, \alpha]$

$$
\begin{aligned}
& L\left(s, x^{k}\right)\left(z_{s}^{k, h}, h^{\theta}, h^{\xi}\right)-L(s, x)\left(z_{s}^{h}+w_{s}^{h, h_{k}}, h^{\theta}, h^{\xi}\right)-B(s)\left\langle\left(z_{s}^{h}, h^{\theta}, h^{\xi}\right),\left(z_{s}^{h_{k}}, h_{k}^{\theta}, h_{k}^{\xi}\right)\right\rangle \\
& =D_{2} f\left(\mathbf{v}^{k}(s)\right) z_{s}^{k, h}-D_{2} f(\mathbf{v}(s)) z_{s}^{k, h}+D_{2} f(\mathbf{v}(s))\left(z_{s}^{k, h}-z_{s}^{h}-w_{s}^{h, h_{k}}\right) \\
& +D_{3} f\left(\mathbf{v}^{k}(s)\right) E^{k}\left(s, z_{s}^{k, h}, h^{\xi}\right)-D_{3} f(\mathbf{v}(s)) E^{k}\left(s, z_{s}^{k, h}, h^{\xi}\right) \\
& +D_{3} f(\mathbf{v}(s))\left(E^{k}\left(s, z_{s}^{k, h}, h^{\xi}\right)-E\left(s, z_{s}^{h}, h^{\xi}\right)\right)+D_{4} f\left(\mathbf{v}^{k}(s)\right) h^{\theta}-D_{4} f(\mathbf{v}(s)) h^{\theta} \\
& -D_{3} f(\mathbf{v}(s)) E\left(s, w_{s}^{h, h_{k}}, 0\right)-B(s)\left\langle\left(z_{s}^{h}, h^{\theta}, h^{\xi}\right),\left(z_{s}^{h_{k}}, h_{k}^{\theta}, h_{k}^{\xi}\right)\right\rangle \\
& =D_{2} f\left(\mathbf{v}^{k}(s)\right) z_{s}^{k, h}-D_{2} f(\mathbf{v}(s)) z_{s}^{k, h}-D_{22} f(\mathbf{v}(s))\left\langle z_{s}^{k, h}, x_{s}^{k}-x_{s}\right\rangle \\
& -D_{23} f(\mathbf{v}(s))\left\langle z_{s}^{k, h}, x^{k}\left(u^{k}(s)\right)-x(u(s))\right\rangle-D_{24} f(\mathbf{v}(s))\left\langle z_{s}^{k, h}, h_{k}^{\theta}\right\rangle \\
& +D_{2} f(\mathbf{v}(s)) q_{s}^{k, h}+D_{22} f(\mathbf{v}(s))\left\langle z_{s}^{k, h}-z_{s}^{h}, x_{s}^{k}-x_{s}\right\rangle+D_{22} f(\mathbf{v}(s))\left\langle z_{s}^{h}, p_{s}^{k}\right\rangle \\
& +D_{23} f(\mathbf{v}(s))\left\langle z_{s}^{k, h}-z_{s}^{h}, x^{k}\left(u^{k}(s)\right)-x(u(s))\right\rangle \\
& +D_{23} f(\mathbf{v}(s))\left\langle z_{s}^{h}, x^{k}\left(u^{k}(s)\right)-x(u(s))-E\left(s, z_{s}^{h_{k}}, h_{k}^{\xi}\right)\right\rangle+D_{24} f(\mathbf{v}(s))\left\langle z_{s}^{k, h}-z_{s}^{h}, h_{k}^{\theta}\right\rangle \\
& +D_{3} f\left(\mathbf{v}^{k}(s)\right) E^{k}\left(s, z_{s}^{k, h}, h^{\xi}\right)-D_{3} f(\mathbf{v}(s)) E^{k}\left(s, z_{s}^{k, h}, h^{\xi}\right) \\
& -D_{32} f(\mathbf{v}(s))\left\langle E^{k}\left(s, z_{s}^{k, h}, h^{\xi}\right), x_{s}^{k}-x_{s}\right\rangle \\
& -D_{33} f(\mathbf{v}(s))\left\langle E^{k}\left(s, z_{s}^{k, h}, h^{\xi}\right), x^{k}\left(u^{k}(s)\right)-x(u(s))\right\rangle-D_{34} f(\mathbf{v}(s))\left\langle E^{k}\left(s, z_{s}^{k, h}, h^{\xi}\right), h_{k}^{\theta}\right\rangle \\
& +D_{32} f(\mathbf{v}(s))\left\langle E^{k}\left(s, z_{s}^{k, h}, h^{\xi}\right)-E\left(s, z_{s}^{h}, h^{\xi}\right), x_{s}^{k}-x_{s}\right\rangle+D_{32} f(\mathbf{v}(s))\left\langle E\left(s, z_{s}^{h}, h^{\xi}\right), p_{s}^{k}\right\rangle \\
& +D_{33} f(\mathbf{v}(s))\left\langle E^{k}\left(s, z_{s}^{k, h}, h^{\xi}\right)-E\left(s, z_{s}^{h}, h^{\xi}\right), x^{k}\left(u^{k}(s)\right)-x(u(s))\right\rangle \\
& +D_{33} f(\mathbf{v}(s))\left\langle E\left(s, z_{s}^{h}, h^{\xi}\right), x^{k}\left(u^{k}(s)\right)-x(u(s))-E\left(s, z_{s}^{h_{k}}, h_{k}^{\xi}\right)\right\rangle \\
& +D_{34} f(\mathbf{v}(s))\left\langle E^{k}\left(s, z_{s}^{k, h}, h^{\xi}\right)-E\left(s, z_{s}^{h}, h^{\xi}\right), h_{k}^{\theta}\right\rangle \\
& +D_{3} f(\mathbf{v}(s))\left[E^{k}\left(s, z_{s}^{k, h}, h^{\xi}\right)-E\left(s, z_{s}^{h}, h^{\xi}\right)-H(s)\left\langle\left(z_{s}^{h}, h^{\xi}\right),\left(z_{s}^{k, h}, h_{k}^{\xi}\right)\right\rangle-E\left(s, w_{s}^{h, h_{k}}, 0\right)\right] \\
& +D_{4}\left(\mathbf{v}^{k}(s)\right) h^{\theta}-D_{4}(\mathbf{v}(s)) h^{\theta}-D_{42} f(\mathbf{v}(s))\left\langle h^{\theta}, x_{s}^{k}-x_{s}\right\rangle \\
& -D_{43} f(\mathbf{v}(s))\left\langle h^{\theta}, x^{k}\left(u^{k}(s)\right)-x(u(s))\right\rangle-D_{44} f(\mathbf{v}(s))\left\langle h^{\theta}, h_{k}^{\theta}\right\rangle \\
& +D_{42} f(\mathbf{v}(s))\left\langle h^{\theta}, p_{s}^{k}\right\rangle+D_{43} f(\mathbf{v}(s))\left\langle h^{\theta}, x^{k}\left(u^{k}(s)\right)-x(u(s))-E\left(s, z_{s}^{h_{k}}, h_{k}^{\xi}\right)\right\rangle,
\end{aligned}
$$


which implies (5.52), using (5.21) and (5.34). Let $L_{3}=L_{3}\left(\alpha, M_{1}, M_{2}, M_{3}\right)$ be defined by (A1) (iv). Then (A1) (iv), (3.2), (4.16), (4.18), (4.25), (5.11) and (5.38) yield

$$
\begin{aligned}
& \int_{0}^{\alpha}\left|g_{5}^{k, h}(s)\right| d s \\
& \leq \quad \alpha L_{3} c_{1, k} N_{1}|h|_{\Gamma} L\left|h_{k}\right|_{\Gamma}+\alpha L_{3} N_{1}|h|_{\Gamma} \max _{s \in[0, \alpha]}\left|p_{s}^{k}\right|_{C}+\alpha L_{3} c_{1, k} N_{1}|h|_{\Gamma} K_{2}\left|h_{k}\right|_{\Gamma} \\
& \quad+L_{3} N_{1}|h|_{\Gamma} \int_{0}^{\alpha}\left|g_{1, k}(s)\right| d s+\alpha L_{3} c_{1, k} N_{1}|h|_{\Gamma}\left|h_{k}\right|_{\Gamma}+\alpha L_{3} K_{8}|h|_{\Gamma} \max _{s \in[0, \alpha]}\left|p_{s}^{k}\right|_{C} \\
& \quad+\alpha L_{3} c_{3, k}|h|_{\Gamma} L\left|h_{k}\right|_{\Gamma}+\alpha L_{3} c_{3, k}|h|_{\Gamma} K_{2}\left|h_{k}\right|_{\Gamma} \\
& \quad+L_{3} K_{8}|h|_{\Gamma} \int_{0}^{\alpha}\left|g_{1, k}(s)\right| d s+L_{1} \int_{0}^{\alpha}\left|g_{3, k}^{h}(s)\right| d s+\alpha L_{3} c_{3, k}|h|_{\Gamma}\left|h_{k}\right|_{\Gamma} \\
& \quad+L_{3}|h|_{\Gamma} \max _{s \in[0, \alpha]}\left|p_{s}^{k}\right|_{C}+L_{3}|h|_{\Gamma} \int_{0}^{\alpha}\left|g_{1, k}(s)\right| d s+\int_{0}^{\alpha}\left|\omega_{D_{2} f}\left(\mathbf{v}(s), \mathbf{v}^{k}(s), z_{s}^{k, h}\right)\right| d s \\
& \quad+\int_{0}^{\alpha}\left|\omega_{D_{3} f}\left(\mathbf{v}(s), \mathbf{v}^{k}(s), E^{k}\left(s, z_{s}^{k, h}, h^{\xi}\right)\right)\right| d s+\int_{0}^{\alpha}\left|\omega_{D_{4} f}\left(\mathbf{v}(s), \mathbf{v}^{k}(s), h_{k}^{\theta}\right)\right| d s
\end{aligned}
$$

Hence $c_{1, k} \rightarrow 0, c_{3, k} \rightarrow 0$ as $k \rightarrow \infty$, (5.18), (5.22), (5.33), (5.49), (5.50) and (5.51) imply (5.53).

Now we are ready to prove the main result of this section.

Theorem 5.17 Assume (A1) (i)-(vi), (A2) (i)-(vii). Then for $t \in[0, \alpha]$ the maps

$$
\Gamma_{2} \supset\left(P_{2} \cap \Gamma_{2}\right) \rightarrow \mathbb{R}^{n}, \quad \gamma \mapsto x(t, \gamma)
$$

and

$$
\Gamma_{2} \supset\left(P_{2} \cap \Gamma_{2}\right) \rightarrow C, \quad \gamma \mapsto x_{t}(\cdot, \gamma)
$$

are twice differentiable wrt $\gamma$ for every $\gamma \in P_{2} \cap \Gamma_{2} \cap \mathcal{P}$, and

$$
D_{22} x(t, \gamma)\langle h, y\rangle=w^{h, y}(t), \quad h, y \in \Gamma_{2},
$$

and

$$
D_{22} x_{t}(\cdot, \gamma)\langle h, y\rangle=w_{t}^{h, y}, \quad h, y \in \Gamma_{2},
$$

where $w^{h, y}$ is the solution of the IVP (5.28)-(5.29). Moreover, if in addition, (A2) (viii) holds, then the maps

$$
\mathbb{R} \times \Gamma_{2} \supset\left([0, \alpha] \times\left(P_{2} \cap \Gamma_{2} \cap \mathcal{P}\right)\right) \rightarrow \mathcal{L}^{2}\left(\Gamma_{2} \times \Gamma_{2}, \mathbb{R}^{n}\right), \quad(t, \gamma) \mapsto D_{22} x(t, \gamma)
$$

and

$$
\mathbb{R} \times \Gamma_{2} \supset\left([0, \alpha] \times\left(P_{2} \cap \Gamma_{2} \cap \mathcal{P}\right)\right) \rightarrow \mathcal{L}^{2}\left(\Gamma_{2} \times \Gamma_{2}, C\right), \quad(t, \gamma) \mapsto D_{22} x_{t}(\cdot, \gamma)
$$

are continuous.

Proof It follows from Theorem 4.7 that $D_{2} x(t, \gamma) \in \mathcal{L}\left(\Gamma, \mathbb{R}^{n}\right)$ exists for all $\gamma \in P_{2}$ and $t \in[0, \alpha]$. Since $|h|_{\Gamma} \leq|h|_{\Gamma_{2}}$ for all $h \in \Gamma_{2}$, it follows that $\left.D_{2} x(t, \gamma)\right|_{\Gamma_{2}} \in \mathcal{L}\left(\Gamma_{2}, \mathbb{R}^{n}\right)$, and $\left.D_{2} x(t, \gamma)\right|_{\Gamma_{2}}$ is the derivtive of the map $\Gamma_{2} \supset\left(P_{2} \cap \Gamma_{2}\right) \rightarrow \mathbb{R}^{n}, \gamma \rightarrow x(t, \gamma)$. For simplicity, the restiction of $D_{2} x(t, \gamma)$ 
to $\Gamma_{2}$ will be denoted by $D_{2} x(t, \gamma)$, as well. Theorem 4.7 yields that $D_{2} x(t, \gamma) h=z(t, \gamma, h)$, where $z(t, \gamma, h)$ is the solution of the IVP (4.13)-(4.14) for $h \in \Gamma_{2}$.

Let $\gamma \in P_{2} \cap \Gamma_{2} \cap \mathcal{P}$ be fixed, $h_{k}=\left(h_{k}^{\varphi}, h_{k}^{\theta}, h_{k}^{\xi}\right) \in \Gamma_{2}(k \in \mathbb{N})$ be a sequence such that $\gamma+h_{k} \in P_{2}$ for $k \in \mathbb{N}, 0 \neq h=\left(h^{\varphi}, h^{\theta}, h^{\xi}\right) \in \Gamma_{2}$. Let $x(t):=x(t, \gamma)$ and $x^{k}(t):=x\left(t, \gamma+h_{k}\right)$ be the solutions of the IVP (1.1)-(1.2), $z^{h}(t):=D_{2} x(t, \gamma) h$ and $z^{k, h}(t):=D_{2} x\left(t, \gamma+h_{k}\right) h$ be the solution of the IVP (4.13)-(4.14), and $w^{h, h_{k}}(t)$ be the solution of the IVP (5.28)-(5.29) corresponding to parameters $h$ and $h_{k}$. Then we have for $t \in[0, \alpha]$

$$
\begin{aligned}
z^{k, h}(t) & =h^{\varphi}(0)+\int_{0}^{t} L\left(s, x^{k}\right)\left(z_{s}^{k, h}, h^{\theta}, h^{\xi}\right) d s \\
z^{h}(t) & =h^{\varphi}(0)+\int_{0}^{t} L(s, x)\left(z_{s}^{h}, h^{\theta}, h^{\xi}\right) d s \\
w^{h, h_{k}}(t) & =\int_{0}^{t}\left(L(s, x)\left(w_{s}^{h, h_{k}}, 0,0\right)+B(s)\left\langle\left(z_{s}^{h}, h^{\theta}, h^{\xi}\right),\left(z_{s}^{h_{k}}, h_{k}^{\theta}, h_{k}^{\xi}\right)\right\rangle\right) d s .
\end{aligned}
$$

Hence Lemma 5.16 and the definition of $q^{k, h}$ give

$$
\begin{aligned}
q^{k, h}(t)= & \int_{0}^{t}\left(L\left(s, x^{k}\right)\left(z_{s}^{k, h}, h^{\theta}, h^{\xi}\right)-L(s, x)\left(z_{s}^{h}+w_{s}^{h, h_{k}}, h^{\theta}, h^{\xi}\right)\right. \\
& \left.\quad-B(s)\left\langle\left(z_{s}^{h}, h^{\theta}, h^{\xi}\right),\left(z_{s}^{h_{k}}, h_{k}^{\theta}, h_{k}^{\xi}\right)\right\rangle\right) d s \\
= & \int_{0}^{t} g_{5}^{k, h}(s) d s+\int_{0}^{t} L(s, x)\left(q_{s}^{k, h}, 0,0\right) d s, \quad t \in[0, \alpha],
\end{aligned}
$$

so (4.11) yields

$$
\left|q^{k, h}(t)\right| \leq \int_{0}^{t}\left|g_{5}^{k, h}(s)\right| d s+\int_{0}^{t}\left|L(s, x)\left(q_{s}^{k, h}, 0,0\right)\right| d s \leq \int_{0}^{\alpha}\left|g_{5}^{k, h}(s)\right| d s+L_{1} N_{0} \int_{0}^{t}\left|q_{s}^{k, h}\right|_{C} d s,
$$

for $t \in[0, \alpha]$. Using that $q^{k, h}(t)=0$ for $t \in[-r, 0]$, Lemma 2.1 implies

$$
\left|q^{k, h}(t)\right| \leq\left|q_{t}^{k, h}\right|_{C} \leq N_{1} \int_{0}^{\alpha}\left|g_{5}^{k, h}(s)\right| d s, \quad t \in[0, \alpha]
$$

where $N_{1}:=e^{L_{1} N_{0} \alpha}$. Therefore (5.53) yields for $t \in[0, \alpha]$

$$
\lim _{k \rightarrow \infty} \sup _{\substack{h \neq 0 \\ h \in \Gamma_{2}}} \frac{\left|q^{k, h}(t)\right|}{|h|_{\Gamma_{2}}\left|h_{k}\right|_{\Gamma_{2}}} \leq \lim _{k \rightarrow \infty} \sup _{\substack{h \neq 0 \\ h \in \Gamma_{2}}} \frac{\left|q_{t}^{k, h}\right|_{C}}{|h|_{\Gamma_{2}}\left|h_{k}\right|_{\Gamma_{2}}} \leq \lim _{k \rightarrow \infty} \sup _{\substack{h \neq 0 \\ h \in \Gamma_{2}}} \frac{N_{1}}{|h|_{\Gamma_{2}}\left|h_{k}\right|_{\Gamma_{2}}} \int_{0}^{\alpha}\left|g_{5}^{k, h}(s)\right| d s=0,
$$

which completes the proof of the second-order differentiability wrt parameters. The continuity of $D_{22} x(t, \gamma)$ follows from Lemma 5.14 .

We note that the method used in this section to prove the existence of the second order derivative $D_{22} x(t, \gamma)$ can not be used to prove the existence of the third order derivative, since some parts of the proof relied on the assumption that the parameter $\gamma$ satisfies the compatibility condition $\gamma \in \mathcal{P}$. The key step to show the existence of higher order derivatives is to get rid of this assumption in the proof of Theorem 5.17. 


\section{References}

[1] M. Brokate and F. Colonius, Linearizing equations with state-dependent delays, Appl. Math. Optim., 21 (1990) 45-52.

[2] Y. Chen, Q. Hu, J. Wu, Second-order differentiability with respect to parameters for differential equations with adaptive delays, Front. Math. China, 5:2 (2010) 221-286.

[3] E. A. Coddington, N. Levinson, Theory of Ordinary Differential Equations, Robert E. Krieger Publishing Company, 1984.

[4] D. L. Cohn, Measure theory, Birkhäuser, 1980.

[5] R.D. Driver, Existence theory for a delay-differential system, Contrib. Differential Equations, 1 (1961) 317-336.

[6] J. K. Hale, L. A. C. Ladeira, Differentiability with respect to delays, J. Diff. Eqns., 92 (1991) 14-26.

[7] J.K. Hale and S.M. Verduyn Lunel, Introduction to Functional Differential Equations, Spingler-Verlag, New York, 1993.

[8] F. Hartung, On classes of functional differential equations with state-dependent delays, Ph.D. Dissertation, University of Texas at Dallas, Richardson, TX, USA, 1995.

[9] F. Hartung, On differentiability of solutions with respect to parameters in a class of functional differential equations, Funct. Differ. Equ., 4:1-2 (1997) 65-79.

[10] F. Hartung, Parameter estimation by quasilinearization in functional differential equations with state-dependent delays: a numerical study, Nonlinear Anal., 47:7 (2001) 4557-4566.

[11] F. Hartung, On differentiability of solutions with respect to parameters in neutral differential equations with state-dependent delays, J. Math. Anal. Appl., 324:1 (2006) 504-524.

[12] F. Hartung, Differentiability of solutions with respect to the initial data in differential equations with state-dependent delays, J. Dynam. Differential Equations, 23:4 (2011) 843884.

[13] F. Hartung, On Differentiability of solutions with respect to parameters in neutral differential equations with state-dependent delays, Ann. Mat. Pura Appl., (2011) DOI 10.1007/s10231-011-0210-5.

[14] F. Hartung, Parameter estimation by quasilinearization in differential equations with statedependent delays, Preprint.

[15] F. Hartung, T. Krisztin, H.O. Walther and J. Wu, Functional differential equations with state-dependent delays: theory and applications, in Handbook of Differential Equations: Ordinary Differential Equations, volume 3, edited by A. Canada, P. Drbek and A. Fonda, Elsevier, North-Holand, 2006, 435-545.

[16] F. Hartung, J. Turi, On differentiability of solutions with respect to parameters in statedependent delay equations, J. Differential Equations 135:2 (1997), 192-237. 
[17] L. A. C. Ladeira, Differentiability with respect to delays for a neutral differential-difference equation, Fields Inst. Commun. 21 (1999), 339-352.

[18] A. Manitius, On the optimal control of systems with a delay depending on state, control, and time. Séminaires IRIA, Analyse et Contrôle de Systèmes, IRIA, France, 1975, 149-198.

[19] B. Slezák, On the parameter-dependence of the solutions of functional differential equations with unbounded state-dependent delay I. The upper-semicontinuity of the resolvent function, Int. J. Qual. Theory Differential Equations Appl., 1:1 (2007) 88-114.

[20] B. Slezák, On the smooth parameter-dependence of the solutions of abstract functional differential equations with state-dependent delay, Functional Differential Equations, 17:3-4 (2010) 253-293.

[21] H.O. Walther, The solution manifold and $C^{1}$-smoothness of solution operators for differential equations with state dependent delay. J. Differential Equations 195 (2003), 46-65.

[22] H.O. Walther, Smoothness properties of semiflows for differential equations with state dependent delay. Russian, in Proceedings of the International Conference on Differential and Functional Differential Equations, Moscow, 2002, vol. 1, pp. 40-55, Moscow State Aviation Institute (MAI), Moscow 2003. English version: J. Math. Sci. 124 (2004), 5193-5207.

[23] H.-O. Walther, Semiflows for neutral equations with state-dependent delays, to appear in Fields Inst. Commun. 